\title{
Endosomal Sorting Drives the Formation of Mutant Prion Endoggresomes
}

Romain Chassefeyre ${ }^{1,2,4,5}$, Tai Chaiamarit ${ }^{1,2,5}$, Adriaan Verhelle $e^{1,2,5}$, Sammy Weiser Novak $^{3}$, Leonardo R. Andrade ${ }^{3}$, Uri Manor ${ }^{3}$, and Sandra E. Encalada ${ }^{1,2, *}$

${ }^{1}$ Department of Molecular Medicine, The Scripps Research Institute, La Jolla, CA 92037, USA

${ }^{2}$ Dorris Neuroscience Center, The Scripps Research Institute, La Jolla, CA 92037, USA ${ }^{3}$ Waitt Advanced Biophotonics Center, The Salk Institute for Biological Studies, La Jolla, CA 92037, USA

${ }^{4}$ Present address: Evotec, 31036 Toulouse, France

${ }^{5}$ These authors contributed equally

* Correspondence: encalada@scripps.edu (S.E.E.) 


\begin{abstract}
Intra-axonal misfolded protein aggregates are a pathological feature of neurodegenerative diseases. How aggregates are formed and cleared is key to maintaining proteostasis. By systematically analyzing the trafficking itinerary of a misfolded GPI-anchored prion protein (PrP) mutant, we unveil endocytic pathways that drive its immediate degradation in the soma, versus its aggregation in axons inside endosomal structures we termed endoggresomes. Axonal sorting occurs post-Golgi, by association of mutant PrP vesicles with Arl8b/kinesin-1/HOPS, a complex that earmarks them for axonal entry, fusion, and aggregation via a mechanism of axonal rapid endosomal sorting and transport-dependent agggregation (ARESTA). Endoggresomes persist in axons due to transport and lysosomal deficits, impairing calcium dynamics and accelerating neuronal death. Reducing ARESTA inhibits endoggresome formation and circumvents these defects. These data identify the endo-lysosomal system as critical for the sorting of misfolded PrP, and ARESTA as an actionable anti-aggregation target that can ameliorate axonal dysfunction in the prionopathies.
\end{abstract}




\section{INTRODUCTION}

The progressive accumulation of misfolded proteins as intra-neuronal aggregate inclusions is a hallmark of neurodegenerative disorders including Alzheimer's Disease (AD), Parkinson's Disease (PD), Huntington's Disease (HD), and the prion diseases ${ }^{1}$. Axons are particularly vulnerable to the formation of aggregates, which are often observed at sites of axonal swellings, and can be formed from wild-type (WT) or mutant misfolded proteins that lose solubility and become cytotoxic to the neuron and also to neighboring cells via spreading ${ }^{2}$. Despite the importance of aggregates in neurodegeneration, the molecular mechanisms and pathways that tip the balance between protein misfolding versus aggregate clearance resulting in the formation of aggregate inclusions in axons, remain undefined.

To regulate function, polarized neurons rely largely on active microtubule-based transport to localize components to distal axonal domains via kinesin-mediated anterograde movement ${ }^{3}$. As the axon has important but limited lysosomal degradative capacity $^{4}$, long-distance dynein-dependent retrograde transport of axonal cargoes back to the lysosome-enriched soma is critical for their efficient degradation ${ }^{5,6}$. Neuronal homeostasis also depends on an interconverting endo-lysosomal system that spreads over long axonal distances, to regulate sorting, signaling, and metabolic functions ${ }^{7}$. How dysregulation of these systems results in aggregate formation in axons remains unclear. Disruptions to protein quality control and ubiquitin-proteasome/autophagosomelysosome clearance pathways can lead to the sequestration of misfolded cytosolic proteins such as tau, $\alpha$-synuclein, TDP-43, FUS, and cytosolic yeast prions, into membrane-free aggresomes, insoluble protein deposits (IPODs), or juxtanuclear quality control (JUNQ) structures ${ }^{8-14}$. Important mechanistic insights on the formation of these cytosolic inclusions have been revealed ${ }^{9,15,16}$. However, compelling evidence suggests that accumulation of pathogenic proteins can also occur within the endo-lysosomal 
system, as several aggregate-prone proteins transit through this system in neurons during processing, and/or as they accumulate within vesicular compartments ${ }^{17}$. How endomembrane proteins such as glycosylphosphatidylinositol (GPI)-anchored proteins form intra-neuronal aggregate inclusions, remains virtually unexplored.

The cellular prion protein $\left(\mathrm{PrP}^{\mathrm{C}}\right.$-cellular or $\left.\mathrm{PrP}{ }^{\mathrm{WT}}\right)$ is a secreted $\mathrm{GPI}$-anchored protein that when misfolded acquires a partially proteinase K-resistant conformation called $\operatorname{PrP}$ scrapie $\left(\mathrm{PrP}^{\mathrm{Sc}}\right)$, which is associated with toxic pathologies in prion disorders ${ }^{18-}$ 24 . While mostly sporadic, some prion diseases are transmissible, and approximately 10$15 \%$ are caused by mutations in the $\operatorname{PrP}$ gene, $P R N P^{25,26}$. Intraneuronal aggregate inclusions have been observed in patient brains with familial PrP mutations, and intraaxonal aggregates have been observed in cellular and mouse familial models of prion disease, including one harboring a nine octapeptide repeat insertion called PrP PG14 shown to associate with dementia and ataxia ${ }^{26-36}$. Where and how PrP aggregates form within neurons, remains unclear. $\mathrm{PrP}^{\mathrm{Sc}}$ has been reported to form aggresomes following proteasome inhibition and after retrotranslocation to the cytosol ${ }^{37}$. However, misfolded PrP has also been observed to accumulate within membrane compartments, pointing toward a role for endosomal trafficking in its aggregation ${ }^{38}$. Indeed, the membrane association and secretion of PrP via its GPI anchor and signal peptide indicate that sorting and trafficking are central to PrP physiology and pathology ${ }^{39}$. In its native or nonnative forms PrP traffics to the cell surface from the ER via the Golgi in the lumen of membrane compartments, from where it is endocytosed back for recycling in endosomes, or transits to lysosomes for degradation ${ }^{18,39-46}$. In non-neuronal cells, this pathway called rapid ER stress-induced export (RESET), was shown to activate within minutes of ER stress to ensure the rapid lysosomal clearance of virtually all misfolded mutant PrP particles ${ }^{47,48}$. It is unknown whether neurons utilize RESET or other mechanisms to clear misfolded PrP, but its accumulation in axons ${ }^{49}$ suggests that it can 
escape clearance in the soma. Consistent with this possibility, our earlier work shows the active transport of post-Golgi $\operatorname{PrP}^{\mathrm{WT}}$ vesicles in and out of the axon by kinesin and dynein ${ }^{50}$, where it may undergo further sorting.

The trafficking and sorting of endo-lysosomes is orchestrated largely by Arl8b, an Arf-like (Arl) family small GTPase that recruits kinesin, and the multi-unit homotypic fusion and protein sorting (HOPS) complex onto late endosomes (LEs), to drive their motility to the cell periphery and towards homotypic fusion with other LEs ${ }^{51-55}$. Arl8b depletion leads to perinuclear lysosome clustering that has been associated with reduced levels of pathogenic proteins in non-neuronal cells ${ }^{56,57}$, but whether and how Arl8b could regulate aggregate formation in axons is unclear. In this study, by systematically analyzing the trafficking itineraries of the misfolded $\mathrm{PrP}^{\mathrm{PG} 14}$ mutant in mammalian neurons, we unveil endo-lysosomal pathways that impose dramatically different fates on mutant PrP: one drives it for immediate degradation within lysosomes in the soma, while the other shuttles mutant PrP into the axon for aggregation in endoggresomes, a new type of aggregate structure that forms inside endosomal compartments and is associated with neuronal toxicity. Endoggresome formation in axons occurs as a result of the association of Arl8b/kinesin-1/HOPS with Golgi-derived mutant PrP vesicles, sorting them for axonal entry, homotypic fusion, and aggregation via a mechanism we call axxonal rapid endosomal sorting and transport-dependent aggregation (ARESTA). Modulating ARESTA inhibits endoggresome formation and prevents neuronal dysfunction, providing an anti-aggregation target that can modulate neuronal dysfunction in the prionopathies. 


\section{RESULTS}

\section{Stress-induced lysosomal clearance of a prion protein mutant in mammalian}

\section{neurons}

Formation of intracellular inclusions could result from an imbalance of synthesis versus clearance pathways. In non-neuronal cells, misfolded GPI-anchored PrP undergoes clearance in lysosomes following cell surface access within minutes of ER stress via RESET $^{47}$. To test whether misfolded $\mathrm{PrP}^{\mathrm{PG} 14}$ is degraded in neurons following this itinerary, we established an in cellulo system in dissociated primary murine hippocampal neuronal cultures by transiently expressing fluorescently (mCherry [mCh], EGFP, or mTagBFP2)-tagged or untagged $\operatorname{PrP}^{\mathrm{PG} 14}$ fusions (or $\mathrm{PrP}^{\mathrm{WT}}$ as control; Fig. 1a), shown previously to be properly processed in vivo ${ }^{27,36,58-60}$. Expression of PrP was under the MoPrP.Xho promoter ${ }^{61}$, and contained the endogenous PrP secretory signal sequence (SS) and GPI anchor sequence to ensure proper translocation to the ER and delivery to the plasma membrane, respectively ${ }^{62}$. Consistent with earlier reports ${ }^{36,63}, \mathrm{PrP}^{\mathrm{PG} 14}-\mathrm{mCh}$ expressed in Neuro-2a (N2a) cells exhibited mild resistance to proteinase $\mathrm{K}(\mathrm{PK})$ as compared to $\mathrm{PrP}^{\mathrm{WT}}$-mCh, suggesting that this mutant partly and constitutively misfolded and misassembled upon transient expression (Supplementary Fig. 1a).

To determine whether $\mathrm{PrP}^{\mathrm{PG} 14}$ follows RESET for clearance in neurons, we tested for four characteristic features upon PrP ${ }^{P G 14}$-mCh expression: (1) increased ER stress; (2) ER-to-Golgi PrP ${ }^{P G 14}$-mCh release; (3) transient PrPPG14-mCh access to the cell surface, followed by (4) $\mathrm{PrP}^{\mathrm{PG} 14}-\mathrm{mCh}$ targeting to lysosomes for degradation. First, co-expression of PrP ${ }^{\mathrm{PG} 14}$-EGFP and an ER stress transcriptional reporter (ERSE-mCh) ${ }^{64}$ resulted in significantly increased signal compared to that observed in neurons expressing either soluble EGFP or PrP ${ }^{W T}$-EGFP (Supplementary Fig. 1b), indicating constitutive ER stress activation. Next, we tested for ER-to-Golgi transit by imaging neurons treated or not with brefeldin A (BFA), an inhibitor of the secretory pathway ${ }^{65}$. 
Following BFA treatment, $\mathrm{PrP}^{\mathrm{PG} 14}-\mathrm{mCh}$ was retained in the ER for at least 6 hours (Fig. 1b and Supplementary Fig. 1c). However, imaging of untreated neurons or of those fixed at various time points post-BFA wash showed rapid, efficient, and successive translocation of misfolded PrP ${ }^{\mathrm{PG} 14}-\mathrm{mCh}$ from the ER to the Golgi, and to compartments positive for Cathepsin B (Cat B), an acidic hydrolase required for lysosomal substrate degradation (Fig. 1b and Supplementary Fig. 1c). PrP ${ }^{\mathrm{PG} 14}-\mathrm{mCh}$ was highly colocalized with puncta positive for Cat B within 2 hours of its expression in untreated neurons (Fig. 1b), indicating that ER export occurred within an acute time period shortly after PrP PG14_ $\mathrm{mCh}$ expression, consistent with non-neuronal RESET dynamics ${ }^{47}$.

To next test whether misfolded $\mathrm{PrP}^{\mathrm{PG} 14}$ transiently accessed the cell surface prior to internalization into LEs, neurons were transfected with $\mathrm{PrP}^{\mathrm{WT}}$ - or $\mathrm{PrP}^{\mathrm{PG} 14}$-mCh fusion constructs containing a Bungarotoxin Binding Sequence (BBS; referred to as PrP ${ }^{W T}$ - or PrP ${ }^{P G 14}-m$ Ch-BBS; Supplementary Fig. 1d), and treated in the media with AlexaFluor 647-labeled bungarotoxin (BTX) (BTX-a647; Fig. 1c) ${ }^{66}$. Live imaging showed colocalization of PrP ${ }^{\mathrm{PG} 14}$-mCh-BBS puncta with BTX-a647 and with LAMP1-EGFP, a LE/lysosomal resident transmembrane protein (Fig. 1c), indicating internalization of cellsurface labeled PrP ${ }^{\mathrm{PG} 14}$-mCh-BBS into LEs. This labeling was specific, as neurons transfected with $\mathrm{PrP}^{\mathrm{PG} 14}$-fusion constructs without the BBS tag did not reveal any BTXa647 signal (Supplementary Fig. 1e). Presence of $\mathrm{PrP}^{\mathrm{PG} 14}-\mathrm{mCh}$ at the cell surface was confirmed by immunofluorescence of fixed unpermeabilized neurons with an antibody against mCh (Supplementary Fig. 1f). Consistent with an earlier report ${ }^{67}$, $\mathrm{PrP}^{\mathrm{PG} 14}-\mathrm{mCh}$ membrane signal was weaker than that of $\mathrm{PrP}^{\mathrm{WT}}-\mathrm{mCh}$, suggesting the limited delivery of this mutant to the cell surface, or a short residence time there prior to internalization ${ }^{48}$.

Colocalization of $\mathrm{PrP}^{\mathrm{PG} 14}-\mathrm{mCh}$ with Cat $\mathrm{B}$ indicates its residence in degradative compartments. To further test whether $\mathrm{PrP}^{\mathrm{PG} 14}$ was actively degraded, we imaged neurons expressing PrP ${ }^{P G 14}$-mTagBFP2 and LAMP1-EGFP with various acidic and 
degradative organelle markers. We observed colocalization of PrP ${ }^{P G 14}-m T a g B F P 2$ signal with the acidic-organelle dye LysoTracker and with Magic Red, a fluorogenic substrate that activates upon cleavage by active Cat $^{68}$ (Fig. 1d). Furthermore, live-imaging showed the active disappearance of $\mathrm{PrP}^{\mathrm{PG} 14}-\mathrm{mCh}$ puncta that colocalized with LAMP1EGFP, indicating active degradation (Supplementary Fig. 1g and Video 1). Altogether, these experiments demonstrate that following ER-stress and a transient excursion to the cell surface, ER export is a pathway involved in the secretion and clearance of misfolded $\mathrm{PrP}^{\mathrm{PG} 14}$ in lysosomes in the neuronal soma, similar to RESET in non-neuronal cells ${ }^{47}$.

\section{Misfolded PrP ${ }^{\mathrm{PG} 14}$ forms persistent intra-axonal aggregate inclusions}

Having shown that misfolded $\mathrm{PrP}^{\mathrm{PG} 14}$ can be cleared from the neuronal soma (Fig. 1), it was unclear how intra-axonal inclusions could form and/or persist in axons, as previously reported ${ }^{36}$. To investigate the fate of axonal misfolded $\operatorname{PrP}^{\mathrm{PG} 14}$, we first determined the localization of $\mathrm{PrP}^{\mathrm{PG} 14}$ in axons. Consistent with previous reports showing $\mathrm{PrP}^{\mathrm{PG} 14}$ limited localization at the cell surface ${ }^{67}$, and with observations in the soma (Fig. 1c), immunofluorescence analysis with or without permeabilization using an anti-PrP antibody (D13) showed scarce PrP ${ }^{\mathrm{PG} 14}-\mathrm{mCh}$ signal at the axonal cell surface compared to $\mathrm{PrP}^{\mathrm{WT}}$-mCh (Fig. 2a), suggesting that misfolded $\mathrm{PrP}^{\mathrm{PG} 14}$-mCh was either not efficiently delivered to the axonal membrane, or that it is only transiently localized there. We next tested whether intra-axonal inclusions formed in axons of cultured primary neurons transiently expressing $\mathrm{PrP}^{\mathrm{PG} 14}$. Live imaging showed prominent inclusions (defined in Methods), one day after PrP ${ }^{\mathrm{PG} 14}$-mCh transfection, and these increased over time (Fig. 2b,c). In contrast, inclusions were largely absent in axons of neurons expressing $\mathrm{PrP}^{\mathrm{WT}}$-mCh. Co-expression drove the accumulation of $\mathrm{PrP}^{\mathrm{WT}}-\mathrm{mCh}$ with PrP ${ }^{P G 14}$-EGFP-inclusions, whereas no accumulations were observed in axons of neurons co-transfected with $\mathrm{PrP}^{\mathrm{WT}}$-EGFP and $\mathrm{PrP}^{\mathrm{WT}}$-mCh (Supplementary Fig. 2a). 
These observations suggest that PrP ${ }^{P G 14}$-EGFP promotes the coalescence of PrP ${ }^{W T}$ $\mathrm{mCh}$, and/or that misfolded $\mathrm{PrP}^{\mathrm{PG} 14}$-EGFP molecules might act as seeds to induce a conformational conversion of $\mathrm{PrP}^{\mathrm{WT}}$-mCh to promote its aggregation. Together with observations showing partial PrP ${ }^{\mathrm{PG} 14}$-mCh PK resistance (Supplementary Fig. 1a), these data suggest that misfolded $\mathrm{PrP}^{\mathrm{PG} 14}$ forms aggregates in axons, thus we henceforth refer to these as aggregate inclusions or aggregates.

To further characterize mutant PrP aggregates, we did correlative fluorescence and scanning electron microscopy (SEM), and observed that they localized to axonal dystrophic swellings averaging $1.57 \pm 0.13 \mu \mathrm{m}$ and $0.54 \pm 0.05 \mu \mathrm{m}$ in length and width, respectively (Fig. 2d and Supplementary Fig. 2b,c). Aggregates also formed to the same degree in axons of neurons expressing untagged $\mathrm{PrP}^{\mathrm{PG} 14}$ or $\mathrm{PrP}^{\mathrm{PG} 14}$-EGFP (Supplementary Fig. 2d,e), in differentiated N2a cells (Supplementary Fig. 2f), and in neurons expressing $\mathrm{PrP}^{\mathrm{D} 177 \mathrm{~N}(\mathrm{M} 128)}$-mCh (Fig. 2e,f), a point mutation (D178N/M129 in humans) that causes fatal familial insomnia, a human prion disease ${ }^{69}$. Altogether, these results indicate that while $\mathrm{PrP}^{\mathrm{PG} 14}$ is degraded in the soma (Fig. 1), it forms aggregates at axonal swellings sites that persist over time, suggesting the presence of mechanisms that actively promote aggregate formation and/or of those that impair their clearance.

\section{Post-Golgi PrP ${ }^{\mathrm{PG} 14}$ vesicles and aggregates are transported and accumulate within endo-lysosomal compartments in axons}

In addition to large aggregates, live imaging using pseudo-total internal reflection fluorescence microscopy (TIRFM) revealed the active bidirectional transport of PrP PG14_ $\mathrm{mCh}$ vesicles in axons (Fig. 3a and Videos 2,3; vesicles versus aggregates defined in Methods). These observations suggested the possibility that $\mathrm{PrP}^{\mathrm{PG} 14}-\mathrm{mCh}$ vesicles transiting within the secretory/endosomal system contributed to the formation of larger aggregates. To test this, we used high-resolution single-particle image analysis ${ }^{70}$ to first 
characterize the dynamics of $\mathrm{PrP}^{\mathrm{PG} 14}$ vesicle transport in axons. The majority of motile $\mathrm{PrP}^{\mathrm{PG} 14}-\mathrm{mCh}$ vesicles exhibited an anterograde movement bias similar to $\mathrm{PrP}^{\mathrm{WT}}-\mathrm{mCh}$ vesicles (Supplementary Fig.3a and Movie 2), and of previously characterized YFP$\operatorname{PrP}^{\mathrm{C}}$ vesicles ${ }^{50}$, mobilizing towards the synapse with fast velocities consistent with kinesin-mediated transport (Fig. 3a,b and Supplementary Fig. 3b). In contrast, all $\mathrm{PrP}^{\mathrm{PG} 14}$-mCh aggregates remained largely stationary, or moved with net velocities that were an order of magnitude (20-50 times) slower $(0.01-0.5 \mu \mathrm{m} / \mathrm{sec})$ than those of vesicles (1-4 $\mu \mathrm{m} / \mathrm{sec})$ (Fig. 3a,b and Supplementary Fig. 3b), suggesting that aggregates and vesicles are two differentially transported cargo populations.

To identify the type of compartment containing $\mathrm{PrP}^{\mathrm{PG} 14}$, we tracked the trajectories of $\mathrm{PrP}^{\mathrm{PG} 14}$ vesicles and aggregates together with those of various secretory and endo-lysosomal cargoes. Live time-lapse showed significant cotransport of PrP ${ }^{\mathrm{PG} 14}$ fast-moving vesicles with a Golgi-derived vesicle marker EGFP-Neuropeptide Y (NPY) ${ }^{71}$, and of vesicles and larger aggregates with EGFP-labeled Rab5, Rab11, Rab7, and LAMP1, markers for early, recycling, LEs, and endo-lysosomes, respectively (Fig. 3c,d and Supplementary Fig. 3c). Both vesicles as well as large aggregates cotransported and/or colocalized with endo-lysosomal markers, consistent with the notion that PrPPG14 traffics and accumulates within endomembranes. To further probe this possibility, we imaged the live time-lapse transport of a photoactivatable (PA) $\operatorname{PrP}^{\mathrm{PG} 14}-\mathrm{mCh}$ fusion following irradiation of the neuronal soma with a $405 \mathrm{~nm}$ laser, in neurons also expressing EGFP-Rab7. We observed the rapid transition of $\mathrm{PrP}^{\mathrm{PG} 14}-\mathrm{PAmCh}$ from the Golgi to EGFP-Rab7-positive LEs, including to large aggregate structures, within 60 minutes post-photoactivation (Supplementary Fig. 3d). To directly test whether PrP PG14 aggregates localized within endomembranes we performed correlative light microscopy and serial or single cross-section scanning electron microscopy (S3EM) of aggregate versus non-aggregate regions. Volumetric 3D reconstructions of serial sections of 
aggregate sites allowed the accurate mapping of $\mathrm{PrP}^{\mathrm{PG} 14}-\mathrm{mCh}$ signal to structures in the S3EM images (Supplementary Fig. 3e). Compared to non-aggregate regions, crosssections that mapped to $\mathrm{PrP}^{\mathrm{PG} 14}$-mCh-positive aggregate swelling sites revealed large electron-dense structures that resided within membrane compartments resembling endosomes (Fig. 3e). Collectively, these observations indicate that $\mathrm{PrP}^{\mathrm{PG} 14}$ is actively transported in Golgi-derived endosomal compartments. In the axon, $\mathrm{PrP}^{\mathrm{PG} 14}$ can accumulate inside larger aggregate endomembrane structures that we name the endoggresome.

\section{Kinesin-1-mediated anterograde transport of $\mathrm{PrP}^{\mathrm{PG} 14}$ endo-lysosomal compartments drives endoggresome formation in axons}

To probe into the origin of endoggresomes, and to test whether $\mathrm{PrP}^{\mathrm{PG} 14}$ vesicles contribute to their formation in axons, we compared the transport dynamics of PrP ${ }^{\mathrm{PG} 14}$ EGFP vesicles and aggregates in wild-type (WT) axons and those from kinesin-1C (KIF5C) knockout $\left(\mathrm{Kif5}^{-/-}\right)$mice $^{50}$, a motor we showed is required for the anterograde transport of YFP-PrP ${ }^{C}$ vesicles in axons ${ }^{50}$. Depletion of KIF5C resulted in an overall impairment of anterograde vesicle dynamics including a significant reduction in the anterograde flux of $\mathrm{PrP}^{\mathrm{PG} 14}$-EGFP vesicles into the axon, and in decreased vesicle densities (Fig. 4a-c and Supplementary Fig. 4a). Moreover, more PrP ${ }^{P G 14}$-EGFP vesicles were stationary at the expense of those moving in the anterograde direction (Fig. 4d). Vesicles also spent less time moving toward the synapse, moved slower, and paused for longer periods during an anterograde run (Fig. 4e and Supplementary Fig. 4b,c). Overall, retrograde PrP ${ }^{\mathrm{PG} 14}$-EGFP vesicle dynamics were impaired to a lesser extent in $\mathrm{Kif5}^{--}$axons, and these vesicles spend more time moving toward the soma (Fig. 4e). These data indicate that KIF5C drives the anterograde flux of $\operatorname{PrP}^{P G 14}$-EGFP vesicles from the soma into the axon. 
To next investigate whether active $\mathrm{PrP}^{\mathrm{PG} 14}-\mathrm{mCh}$ vesicle transport contributed to endoggresome formation, we quantitated $\mathrm{PrP}^{\mathrm{PG} 14}$-mCh aggregate densities in $\mathrm{Kif5}^{-/-}$ axons. Strikingly, 2 days-post $\mathrm{PrP}^{\mathrm{PG} 14}-\mathrm{mCh}$ transfection, aggregate densities were significantly decreased in $\mathrm{Kif5}^{-/ /}$compared to WT hippocampal axons (Fig. 4f,g), and these levels were sustained for at least 10 days (Supplementary Figure 4d,e). Overexpression of EGFP-KIF5C in Kif5c-- neurons transfected with $\mathrm{PrP}^{\mathrm{PG} 14}-\mathrm{mCh}$ restored higher aggregate densities, showing that the decreased densities were due to the specific removal of KIF5C (Supplementary Fig. 4f,g). To further test the role of kinesin-1, we quantitated aggregate densities in axons of cultured hippocampal neurons from kinesin light chain $1(\mathrm{KLC} 1) \mathrm{KO}\left(\mathrm{Klc}^{-/-}\right)$mice, and from conditional kinesin-1B (Kif5b) KO mice ${ }^{72}$, both previously implicated in the anterograde transport of YFP-PrP ${ }^{\mathrm{C}}$ vesicles $^{50}$. PrP ${ }^{\mathrm{PG} 14}$-mCh aggregate densities were also significantly decreased in $\mathrm{KIc1}^{-1-}$ neurons, and in those of conditional Kif5b KO neurons in a cre-adenovirus $\mathrm{MOI}$ dosedependent manner (Supplementary Fig. 4h-I). Notably, aggregate densities in axons of Kif5c $c^{-/-}$neurons expressing $\operatorname{PrP}^{\mathrm{D} 177 \mathrm{~N}(\mathrm{M} 128)}-\mathrm{mCh}$ were also significantly lower

(Supplementary Fig. 4m,n), indicating a general requirement of kinesin-1 for generating intra-axonal endoggresomes. To test whether decreased aggregate densities were the result of generalized transport impairments, we reduced the function of the main neuronal retrograde motor cytoplasmic dynein heavy chain 1 (DHC1), using previously validated shRNAs ${ }^{50}$. Despite impairing $\mathrm{PrP}^{\mathrm{PG} 14}-\mathrm{mCh}$ vesicle transport, and thus identifying DHC1 as a retrograde motor for these vesicles (Supplementary Fig. 4o), reducing DHC1 did not alter PrP ${ }^{\mathrm{PG} 14}$-EGFP aggregate densities (Fig. 4f,g). Collectively, these data reveal that in addition to mediating the anterograde flux of $\mathrm{PrP}^{\mathrm{PG} 14}$ vesicles, kinesin-1-mediated anterograde transport is required to form endoggresomes in axons.

\section{Transient access of misfolded PrP ${ }^{\mathrm{PG} 14}$ to the cell surface along axons is required}




\section{for endoggresome formation}

To further probe the mechanism of endoggresome formation, we characterized the Golgi-to-endosome sorting itineraries of $\mathrm{PrP}^{\mathrm{PG} 14}-\mathrm{mCh}$ particles in axons. Specifically, we tested whether upon entering the axon, $\mathrm{PrP}^{\mathrm{PG} 14}-\mathrm{mCh}$ accessed the plasma membrane en route to aggregation. Neurons expressing $\mathrm{PrP}^{\mathrm{PG} 14}-\mathrm{mCh}-\mathrm{BBS}$ were treated with $\mathrm{BTX}-$ a647 for 10 minutes at $4^{\circ} \mathrm{C}$ to inhibit active endocytosis, and then fixed without permeabilization prior to imaging. BTX-a647 signal colocalized with a subset of PrP PG14 mCh-BBS-positive puncta along axons (Fig. 5a), indicating that the latter accessed the axonal plasma membrane. BTX-a647 labeling was specific, as a647 signal was not observed in neurons expressing $\mathrm{PrP}^{\mathrm{WT}}-\mathrm{mCh}$ or $\mathrm{PrP}^{\mathrm{PG} 14}-\mathrm{mCh}$ without the BBS sequence (Supplementary Fig. 5a). To determine if PrP ${ }^{\mathrm{PG} 14}$ internalized into LEs, we treated $\mathrm{PrP}^{\mathrm{PG} 14-}{ }^{-} \mathrm{mCh}-\mathrm{BBS}$-expressing neurons co-expressing LAMP1-EGFP, with BTX-a647 for 2 hours at $37^{\circ} \mathrm{C}$ prior to live imaging or fixation and permeabilization. We observed extensive colocalization and cotransport between intra-axonal $\mathrm{PrP}^{\mathrm{PG} 14-}{ }^{-} \mathrm{mCh}-\mathrm{BBS}$, LAMP1-EGFP puncta/aggregates, and BTX-a647 signal (Fig. 5b and Supplementary Fig. 5b), indicating $\mathrm{PrP}^{\mathrm{PG} 14-} \mathrm{mCh}-\mathrm{BBS}$ sorted into LEs post-endocytosis. BTX-a647 signal was observed along axons, demonstrating that $\mathrm{PrP}^{\mathrm{PG} 14}$ undergoes dynamic bouts of exo- and endocytosis throughout the axonal surface. Importantly, addition of the BBS sequence did not alter endoggresome densities in axons (Supplementary Fig. 5c). Inhibition of endocytosis with Dynasore, a dynamin GTPase inhibitor ${ }^{73}$ resulted in a significant, albeit partial decrease in internalization of BTX-a647-labeled $\mathrm{PrP}^{\mathrm{PG} 14}$-mCh particles (Supplementary Fig. 5d), suggesting that $\mathrm{PrP}^{\mathrm{PG} 14}$-mCh is partly internalized by clathrindependent pathways.

We next investigated whether PrP ${ }^{\mathrm{PG} 14}$-mCh cell surface targeting and endocytosis was required to form intra-axonal endoggresomes. We quantitated aggregate densities in axons of neurons expressing $\mathrm{PrP}^{\mathrm{PG} 14}-\mathrm{mCh}$ tagged or not with a 
Thrombin Cleavage Sequence (PrP $\left.{ }^{P G 14}-\mathrm{mCh}-\mathrm{TCS}\right)$, and treated or not with 5 units $/ \mathrm{ml}$ of Thrombin protease for 48 hours to cleave PrP-mCh-TCS at the cell surface prior to endocytosis (Supplementary Fig. 5e). Thrombin treatment resulted in significantly reduced densities of $\mathrm{PrP}^{\mathrm{PG} 14}$-mCh-TCS endoggresomes compared to untagged PrP $\mathrm{PG}^{\mathrm{PG}}$ $\mathrm{mCh}$ (Fig. 5c,d), indicating that access to the plasma membrane is required for their formation. We further tested this cell surface requirement by expressing an anchorless $\operatorname{PrP}^{\mathrm{PG} 14}$ double mutant (PrP ${ }^{\mathrm{PG} 14 / \mathrm{S} 232 \mathrm{X}}$-EGFP) that is unable to associate with membranes ${ }^{47}$. While small vesicles carrying this mutant were actively transported in axons, the density of aggregates was largely reduced (Fig. 5e,f). Notably, co-expression with GPI-anchored $\mathrm{PrP}^{\mathrm{PG} 14}$-mCh resulted in $\mathrm{PrP}^{\mathrm{PG} 14 / \mathrm{S} 232 \mathrm{X}}$-EGFP accumulation in large puncta (Supplementary Fig. 5f), suggesting that deleting the GPI anchor did not abolish the propensity of this mutant to either misfold and form aggregates, or to accumulate. Altogether, these data indicate that transient cell surface access of $\mathrm{PrP}^{\mathrm{PG} 14}$ occurs along the length of axons, and is required to form $\mathrm{PrP}^{\mathrm{PG} 14}$-mCh endoggresomes in LEs. As endoggresome formation requires transport-dependent sorting (Fig. 4 and 5), we term

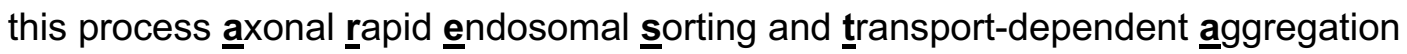
(ARESTA), and distinguish it from RESET, which despite involving a similar $\operatorname{PrP}^{\mathrm{PG} 14}$ cell surface-to-lysosome itinerary, has an opposite role in the soma to clear misfolded $\operatorname{PrP}^{\mathrm{PG} 14}$ (Fig. 1).

\section{Arl8b drives ARESTA-mediated formation of axonal PrP ${ }^{P G 14}$ endoggresomes via recruitment of kinesin-1 and HOPS}

While cell surface ARESTA (henceforth termed "indirect ARESTA"), is required for endoggresome formation (Fig. 5), two observations led us to hypothesize that direct Golgi-to-LE or LE homotypic fusion ${ }^{74-76}$ might also contribute to endoggresome biogenesis in axons. First, $\mathrm{PrP}^{\mathrm{PG} 14}$ particles were internalized from the cell surface in 
single endosomes (Fig. 5b), but over a period of 2-5 days, endoggresomes were observed in enlarged LEs (Fig. $\mathbf{2 b}$ and $\mathbf{3 e}$ ), suggesting fusion of endosomes postendocytosis. Second, inhibiting indirect ARESTA reduced but did not abolish the number of $\mathrm{PrP}^{\mathrm{PG} 14}$ endoggresomes (Fig. 5c,d), suggesting the presence of parallel aggregateforming pathways.

As Arl8b orchestrates the trafficking and fusion of LEs ${ }^{53-56,77,78}$, we investigated whether it contributed to the formation of aggregates via association with $\mathrm{PrP}^{\mathrm{PG} 14}$ endosomal vesicles to direct their homotypic fusion in axons. We first tested whether Arl8b colocalized with $\mathrm{PrP}^{\mathrm{PG} 14}$ vesicles. Live imaging 2 days post-co-expression of Arl8b $b^{W T}-m C h$ and PrP ${ }^{P G 14}-m T a g B F P 2$, showed their colocalization in the soma, and in the cotransport of these vesicles and those carrying ss-NPY-EGFP in the axon, suggesting that Arl8b loaded onto post-Golgi $\mathrm{PrP}^{\mathrm{PG} 14}$ vesicles and entered the axon as a complex (Supplementary Fig. 6a,b). Endogenous Arl8b as recognized with an antiArl8b antibody, also colocalized with $\mathrm{PrP}^{\mathrm{PG} 14}$ vesicles in soma and axons (Supplementary Fig. S8). Furthermore, a majority of $\mathrm{PrP}^{\mathrm{PG} 14}$-mCh vesicles co-migrated

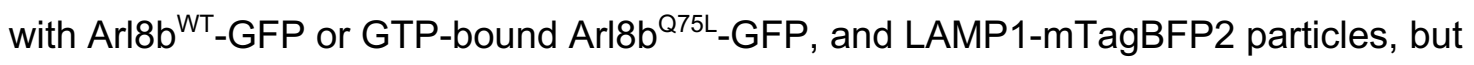

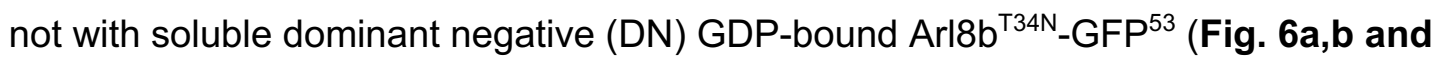
Supplementary Fig. 6c), indicating that GTPase activity is required for Arl8b association with $\mathrm{PrP}^{\mathrm{PG} 14}-\mathrm{mCh}$ vesicles. To next test the role of Arl8b in aggregation, we quantitated endoggresome densities following Arl8b reduction or overexpression. We observed significantly less aggregates following Arl8b knock-down using shRNAs (Supplementary Fig. 6d-f), and densities were lower in neurons expressing untagged GDP-bound Arl8b $b^{\top 34 N}$ (Fig. 6c,d), indicating that Arl8b is required for $\mathrm{PrP}^{\mathrm{PG} 14}-\mathrm{mCh}$ aggregation. In contrast, significantly higher endoggresome densities were observed in

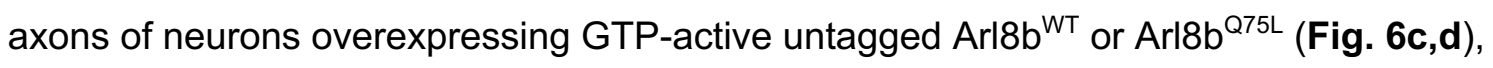
demonstrating that membrane-bound Arl8b is sufficient to drive endoggresome 
formation.

We next examined the mechanistic basis of PrP ${ }^{P G 14}-m C h-A r l 8 b-m e d i a t e d$ aggregate build-up in axons. Arl8b is known to recruit kinesin-1 (KLC1 and KIF5) to LEs via binding of its effector SifA and kinesin-interacting protein (SKIP) $)^{54,79}$, and to directly bind to Vps41, a unit of the HOPS complex required for LE tethering and fusion ${ }^{80}$. We posited that Arl8b drives endoggresome formation by recruiting SKIP, KIF5C, and Vps41 to $\mathrm{PrP}^{\mathrm{PG} 14}$ vesicles, to both propel them anterogradely and to promote their fusion in axons. Live imaging showed extensive cotransport between vesicles carrying PrPPG14_ mTagBFP2, Arl8b ${ }^{\text {WT }}$-mCh, and EGFP-hSKIP (Supplementary Fig.6g-k), and between those carrying $\mathrm{PrP}^{\mathrm{PG} 14}$-mCh and EGFP-Vps41 (Fig. 6e,f). To investigate the role of Vps41 and SKIP in the formation of endoggresomes, we quantitated aggregate densities in axons of neurons treated either with Vps41 siRNAs (Supplementary Fig. 6I-o), or with a SKIP inhibitory peptide that we designed comprising region aa 1-186 (SKIP 1186), previously shown to be required to bridge interactions between Arl8b and kinesin$1^{79}$. Aggregate densities were significantly reduced in axons treated with SKIP 1-186 and Vps41 siRNAs (Fig. 6c,d,g,h). Furthermore, reducing Vps41 resulted in smaller and less stationary $\mathrm{PrP}^{\mathrm{PG} 14}$-mCh vesicles in the soma and axons, suggesting decreased vesicle fusion and restored motility with less aggregates (Fig. 6g and Supplementary Fig. $6 p, q)$.

Next, we tested whether Arl8b recruited kinesin-1 to $\mathrm{PrP}^{\mathrm{PG} 14}$ vesicles to direct their movement into the axon. Overexpression of $A r l 8 b^{W T}-m C h$ resulted in a striking increase in the percentage of $\mathrm{PrP}^{\mathrm{PG} 14}$-mTagBFP2 vesicles and aggregates that cotransported with 2xmCh-KIF5C (Fig. 6i-j), and this recruitment depended on Arl8b GTPase activity (Supplementary Fig. 7a). Moreover, the flux of PrP $P^{P G 14}-m C h$ vesicles was significantly enhanced in axons overexpressing untagged Arl8b, resulting in increased numbers of moving $\mathrm{PrP}^{\mathrm{PG} 14}-\mathrm{mCh}$ vesicles into the axon (Supplementary Fig. 
7b-d). Altogether, these data indicate that GTPase-active Arl8b ${ }^{W T}$ loads onto Golgiderived PrP ${ }^{\mathrm{PG} 14}$ vesicles, recruits SKIP, kinesin-1, and Vps41, and acts as a key driver of the movement and direct fusion of $\mathrm{PrP}^{\mathrm{PG} 14}$ vesicles in the axon, thus contributing towards the biogenesis of PrP $\mathrm{PG}^{\mathrm{P} 14}$ endoggresomes via a process we termed "direct"

\section{ARESTA.}

\section{Direct and indirect ARESTA converge on Arl8b to form axonal endoggresomes}

To investigate whether direct and indirect ARESTA act independently or in concert to generate $\mathrm{PrP}^{\mathrm{PG} 14}$ axonal endoggresomes, we overexpressed Arl8b to promote aggregate formation, while blocking indirect ARESTA by inhibiting the uptake of cell surface PrP ${ }^{\mathrm{PG} 14}-\mathrm{mCh}$. Live imaging of neurons expressing $\mathrm{PrP}^{\mathrm{PG} 14}-\mathrm{mCh}-\mathrm{TCS}$ and simultaneously transfected with Arl8b $\mathrm{b}^{\mathrm{WT}}$-GFP at a time point when axons otherwise exhibited few endoggresomes (day 1 post-PrP ${ }^{\mathrm{PG} 14}$-mCh-TCS transfection), resulted in the same level of increased aggregate densities in axons treated or not with thrombin (Supplementary Fig. 7e,f). These observations indicate that Arl8b ${ }^{W T}$-GFP overexpression alone was sufficient to promote endoggresome formation, bypassing the contribution of cell surface $\mathrm{PrP}^{\mathrm{PG} 14}-\mathrm{mCh}$, and suggest that direct and indirect ARESTA act in parallel.

We next investigated whether Arl8b associated with $\mathrm{PrP}^{\mathrm{PG} 14}$ vesicles endocytosed from the cell surface following indirect ARESTA. Live imaging of axons

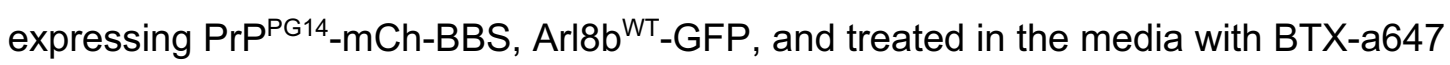
resulted in significant cotransport between these three vesicle populations (Fig. $6 \mathbf{k}, \mathbf{I})$, indicating Arl8b can intersect endocytosed $\mathrm{PrP}^{\mathrm{PG} 14}$-mCh-BBS vesicles. Collectively, these data indicate that direct and indirect ARESTA act in parallel and converge on Arl8b to drive the formation of axonal $\mathrm{PrP}^{\mathrm{PG} 14}$ aggregates. These observations position Arl8b as a key regulator of both the direct fusion of Golgi-to-LE PrP ${ }^{\mathrm{PG} 14}$ vesicles, as well 
as of the endosomal sorting of $\mathrm{PrP}^{\mathrm{PG} 14}$ internalized from the cell surface into endosomes that drives the biogenesis of mutant prion endoggresomes in axons.

\section{Arl8b is a key determinant of $\mathrm{PrP}^{\mathrm{PG} 14}$ axonal entry}

As Arl8b is an endosomal GTPase, we tested whether it associated uniquely with postGolgi endosomal $\mathrm{PrP}^{\mathrm{PG} 14}$ vesicles destined for axonal entry, thus distinguishing these from $\mathrm{PrP}^{\mathrm{PG} 14}$ vesicles secreted in the soma for RESET degradation (Fig. 1). To test this, we followed the ER-to-Golgi-to-LE secretory itinerary of $\mathrm{PrP}^{\mathrm{PG} 14}$-mTagBFP2 (or -mCh) and Arl8b in the soma in neurons treated or not with BFA. To prevent overexpression of Arl8b, we analyzed the localization of endogenous Arl8b in fixed cells using a previously validated antibody ${ }^{4}$. Colocalization analysis showed that as observed earlier (Fig. 1), PrP ${ }^{P G 14}-m T a g B F P 2$ (or -mCh) translocated from the ER to the Golgi, and to LAMP1mTag FBP2-positive endo-lysosomes immediately post-BFA wash (Supplementary Fig. 8a). However, Arl8b did not extensively colocalize in the soma with PrP ${ }^{P G 14}-m T a g B F P 2$ throughout this itinerary, although some colocalization was observed suggesting Arl8b started association with $\mathrm{PrP}^{\mathrm{PG} 14}$ vesicles 2 hrs post-transfection (Supplementary Fig. 8a, i, ii). Instead, colocalization of Arl8b with PrP ${ }^{P G 14}-m C h$ and LAMP1-mTagBFP2 vesicles occurred primarily in the proximal axon, as observed earlier in more distal axonal regions (Fig. 6). As Arl8b associates in the axon with $\mathrm{PrP}^{\mathrm{PG} 14}$ vesicles that directly endocytose from the cell surface (Fig. 6k,j), we investigated whether Arl8b also associated with $\mathrm{PrP}^{\mathrm{PG} 14}$ vesicles that internalized from the somatic cell surface following RESET. Immunofluorescence of neurons co-transfected with PrP ${ }^{\mathrm{PG} 14}$-mCh-BBS and LAMP1-mTagBFP2 and stained with an antibody against Arl8b revealed the colocalization of PrP ${ }^{P G 14}-m C h-B B S$ and LAMP1-mTagBFP2 vesicles that were also positive for BTX-a647 signal, but that were negative for Arl8b (Supplementary Fig. 8b) indicating that unlike in the axon, Arl8b does not recognize $\mathrm{PrP}^{\mathrm{PG} 14}$ vesicles that are 
internalized from the somatic cell surface. Collectively, these data show that Arl8 does not associate with somatic PrP ${ }^{\mathrm{PG} 14}$ vesicles undergoing RESET, but instead uniquely loads onto post-Golgi $\mathrm{PrP}^{\mathrm{PG} 14}$ vesicles that enter the axon, suggesting that Arl8b is a key regulator of $\mathrm{PrP}^{\mathrm{PG} 14}$ axonal entry.

\section{Impaired retrograde transport and lysosomal degradation sustain endoggresomes in axons}

We next investigated how $\mathrm{PrP}^{\mathrm{PG} 14}$ endoggresomes persisted in axons. Previous studies revealed that while limited, the axon has some local degradation capacity ${ }^{4}$, although the bulk of degradation of axonal cargoes occurs primarily via their retrograde long-distance movement back to the soma for fusion with degradative lysosomes ${ }^{5}$. Thus, we tested whether persistence of $\mathrm{PrP}^{\mathrm{PG} 14}$ endoggresomes in axons was due to impairments in local axonal lysosomal degradation capacity, and/or failure of $\mathrm{PrP}^{\mathrm{PG} 14}$ vesicles to undergo retrograde transport. Several experiments suggested deficits in endo-lysosomal maturation and lack of local active axonal $\mathrm{PrP}^{\mathrm{PG} 14}$ degradation. First, axonal PrP ${ }^{\mathrm{PG} 14}$ mCh-positive aggregate densities increased over time compared to $\mathrm{PrP}^{\mathrm{WT}}$-mCh-positive puncta (Fig. 2b,c), suggesting impaired in situ degradation. Additionally, treatment of PrP ${ }^{P G 14}$-mCh-expressing neurons with Bafilomycin A1 (BafA1), an inhibitor of a Iysosomal V-ATPase, a proton pump that acidifies vesicles ${ }^{81}$, did not increase the density of aggregates compared to DMSO-treated controls (Supplementary Fig. 9a). As a positive control, $\mathrm{PrP}^{\mathrm{WT}}$-mCh neurons treated with BafA1 showed a significant increase in aggregate densities compared to DMSO-treated ones, indicating inhibition of acidification (Supplementary Fig. 9a). These data suggest that $\mathrm{PrP}^{\mathrm{PG} 14}$ aggregates reside in endo-lysosomal compartments that are not actively degradative. Consistent with this observation, and in contrast to the soma (Fig. 1d), LysoTracker signal was not readily observed in $\mathrm{PrP}^{\mathrm{PG} 14}$-mCh axons (Fig. 7a,b and Supplementary Fig. 9b), 
showing compromised lysosomal degradative potential. Third, neurons expressing PrP ${ }^{P G 14}$-mCh-BBS showed particles that were actively transported inside axons 24 hours following BTX-a647 treatment and wash-off, indicating that internalized PrP ${ }^{\mathrm{PG} 14}$-mChBBS molecules were not degraded in lysosomes (Fig. 7c).

We next tested whether the retrograde transport of $\operatorname{PrP}^{\mathrm{PG} 14}$ vesicles was impaired. In addition to the largely stationary or slow moving $\mathrm{PrP}^{\mathrm{PG} 14}-\mathrm{mCh}$ endoggresomes (Fig. 3a,b), live imaging and quantitative analysis showed PrP ${ }^{\text {PG14_ }}$ EGFP vesicles also moved significantly slower and with less processivity in the anterograde and retrograde directions compared to $\mathrm{PrP}^{\mathrm{WT}}$-EGFP vesicles

(Supplementary Fig. 9c). To further test whether retrograde transport was impaired, we imaged the transport of LAMP1-EGFP vesicles in $\mathrm{PrP}^{\mathrm{WT}}-\mathrm{mCh}$ versus $\mathrm{PrP}^{\mathrm{PG} 14}$-mCh expressing neurons. The proportion of stationary LAMP1-EGFP particles in PrP ${ }^{P G 14}$ axons was significantly increased, and less vesicles traveled retrogradely, moved slower in the anterograde direction, and spent more time paused and less time moving toward the soma (Fig. $\mathbf{7 d - g}$ ). These results show defective retrograde and anterograde movement of LAMP1-EGFP vesicles, suggesting impaired distribution of axonal LEs in $\mathrm{PrP}^{\mathrm{PG} 14}$ axons. Collectively, our data indicate that $\mathrm{PrP}^{\mathrm{PG} 14}$ vesicles and endoggresomes, as well as LEs are not properly transported toward the soma, nor are they actively degraded in axonal lysosomes, contributing to sustained presence of $\operatorname{PrP}^{\mathrm{PG} 14}$ endoggresomes in axons.

\section{Functional consequences of kinesin-1-dependent formation of PrP ${ }^{\text {PG14 }}$ endoggresomes in axons}

To probe whether axonal $\mathrm{PrP}^{\mathrm{PG} 14}$ endoggresomes lead to neuronal dysfunction, we tested whether the intrinsic capacity of cultured hippocampal neurons expressing 
$\mathrm{PrP}^{\mathrm{PG} 14}$-mCh to intake calcium was compromised upon $\mathrm{KCl}$-induced depolarization, as shown previously for cerebellar granule culture neurons ${ }^{82}$. Cultured primary hippocampal neurons were transduced with $\mathrm{PrP}^{\mathrm{WT}}-\mathrm{mCh}$ or $\mathrm{PrP} \mathrm{PG}^{\mathrm{P}}$-mCh Adeno-associated virus (AAV-DJ), to obtain high transduction efficiencies of $96 \% \pm 2 \%$ and $97 \% \pm 2 \%$ (mean \pm SEM), respectively. Comparable endoggresome densities were observed in transduced versus transfected axons (Supplementary Fig. 10d). Single-cell calcium imaging of AAV-DJ-PrP ${ }^{P G 14}$-mCh-transduced neurons pre-loaded with the calcium sensitive dye Fluo-4 AM resulted in a defective calcium influx in response to $\mathrm{KCl}$-induced depolarization compared to neurons transduced with AAV-DJ-PrP ${ }^{W T}-m C h$ (Fig. 8a-c). We investigated whether reducing endoggresome formation via depletion of ARESTA component kinesin-1 could inhibit calcium intake dynamics. Strikingly, Kif5c ${ }^{-/-}$neurons with significantly less endoggresomes (Fig. 4f,g), but treated with AAV-DJ-PrP ${ }^{P G 14}-m C h$ displayed normal calcium influx dynamics comparable to those of neurons expressing AAV-DJ-PrP'TT-mCh (Fig. 8a-c). Importantly, calcium intake was not altered in control

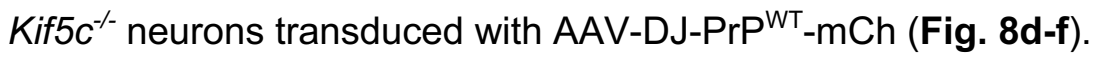

To further characterize the effect of $\mathrm{PrP}^{\mathrm{PG} 14}$ endoggresomes, we quantitated cell death in WT versus $\mathrm{Kif5}^{-/-}$primary hippocampal neurons. At 10 -day post-PrP ${ }^{\mathrm{PG} 14}$-mCh transfection, when endoggresomes are prominent in WT axons (Supplementary Fig. 4d,e), expression of $\mathrm{PrP}^{\mathrm{PG} 14}$-mCh resulted in a $\sim 4.5$-fold increase in cell death compared to non-transfected neurons or those expressing $\mathrm{PrP}^{\mathrm{WT}}-\mathrm{mCh}$ (Supplementary Fig. 10b,c). Notably, neuronal death at 10 days post-transfection was not significantly different in $K i f 5 c^{-/-}$neurons expressing $\mathrm{PrP}^{\mathrm{PG} 14}-\mathrm{mCh}$ compared to those expressing $\mathrm{PrP}^{\mathrm{WT}}-\mathrm{mCh}$ or not transfected, suggesting that reduced endoggresome formation in KIF5C-depleted axons prevented cell death. Altogether, our data indicate that the presence of endoggresomes imparts toxicity and affects neuronal viability, and that their inhibition by depletion of kinesin- 1 abrogates these defects to prevent neuronal 
dysfunction and cell death.

\section{DISCUSSION}

By characterizing the trafficking itineraries of a misfolded GPI-anchored PrP mutant in mammalian neurons, we uncovered novel endo-lysosomal pathways that either sort mutant PrP for immediate degradation within lysosomes in the soma in a process that resembles non-neuronal RESET ${ }^{47,48}$, or that direct mutant PrP into the axon for aggregation via ARESTA inside late endosomal structures which we call endoggresomes. (Supplementary Fig. 11). We uncovered the mechanistic basis of selective endoggresome formation in axons: the small GTPase Arl8b associates with post-Golgi vesicles harboring misfolded mutant PrP, earmarking them for axonal entry. Arl8b recruits kinesin-1 and Vps41(HOPS) and this endosomal vesicle-protein complex moves in the anterograde direction along the axon to undergo homotypic fusion and form aggregates. Endoggresomes persist in axons in response to impaired retrograde transport and decreased axonal degradative capacity, resulting in calcium intake defects and accelerated neuronal death. Remarkably, these defects can be circumvented by inhibiting endoggresome formation through the depletion of the ARESTA component kinesin-1 (Supplementary Fig. 11).

Our findings have a number of important implications for the understanding of quality control mechanisms that neurons have adopted in response to aggregation-prone proteins residing within the secretory pathway. The discovery of a partitioning itinerary for mutant PrP for somatic degradation versus axonal aggregation, was unexpected. In non-neuronal RESET, essentially all misfolded PrP particles were shown to be degraded in lysosomes via a plasma membrane route, indicating this is a bone fide and effective clearance mechanism ${ }^{48}$. In neurons this does not appear to be the case. While the precise ratio of $\mathrm{PrP}^{\mathrm{PG} 14}$ undergoing RESET versus ARESTA is unknown, our data argue 
that mutant PrP can escape immediate degradation and sort into axons. We posit that in neurons, segregation toward degradation via RESET could be triggered by ER stress activation, as proposed for non-neuronal cells ${ }^{48}$. Under this scenario, non-neuronal misfolded PrP was shown to associate with ER cargo receptors and chaperones, which were proposed to tag the complex for travel to the plasma membrane prior to internalization in lysosomes. Our work shows that expression of $\mathrm{PrP}^{\mathrm{PG} 14}$ robustly activates neuronal ER stress, followed by ER-to-Golgi egress of $\mathrm{PrP}^{\mathrm{PG} 14}$ within minutes, and subsequent targeting to degradative lysosomes via the cell surface within an hour (Fig. 1). These dynamics are consistent with non-neuronal RESET, suggesting ER stress could also guide mutant PrP toward secretion and clearance.

Our data provides evidence that sorting of mutant PrP into the axon occurs at the level of the trans-Golgi network (TGN), by engagement of an Arl8b/kinesin/HOPS complex that we propose represents a signature for axonal entry. Arl8b was absent from RESET-bound $\mathrm{PrP}^{\mathrm{PG} 14}$ vesicles, but associated with over $80 \%$ of $\mathrm{PrP}^{\mathrm{PG} 14}$ vesicles entering the axon, suggesting it represents an axonal tag (Fig. 6). Evidence for a Golgito-axon itinerary was also provided by the observation that $\mathrm{PrP}^{\mathrm{PG} 14}$ vesicles co-transport with Arl8b and Golgi-derived markers into the axon, indicating TGN-to-endosome sorting. Both Arl8b and Vps41 have been implicated in directing TGN-to-LE itineraries ${ }^{55}$, so these roles appear to be conserved during their interactions with mutant PrP vesicles. How Arl8b recognizes mutant PrP vesicles at the TGN requires further investigation, but the mechanism could relate to the regulation of endosomal sorting. A plausible participant is BORC (BLOC-One-Related Complex), an Arl8b adaptor that has been shown to couple LEs to Arl8b to regulate their distribution towards the periphery of cells uniquely in axons and not in dendrites ${ }^{68,83}$. Cellular stress is known to trigger a switch from a BORC-Arl8b to a BORC-LAMTOR (late endosomal/lysosomal adaptor and MAPK and mechanistic target of rapamycin [mTOR] activator) association, resulting in the 
perinuclear retention of axonal $\mathrm{LEs}^{84}$. As such, an attractive possibility is that ER stress activation resulting from mutant PrP expression could delineate the choice between the formation of either of these complexes on $\mathrm{PrP}^{\mathrm{PG} 14}$ vesicles at the TGN, to partition them to somatic versus axonal itineraries.

Once in the axon, mutant PrP undergoes further sorting with Arl8b at the helm. The Arl8b/SKIP/kinesin-1/Vps41 complex provides the axon with a steady flux of mutant PrP vesicles that either fuse with the axonal plasma membrane allowing mutant PrP transient access before internalization in LEs (indirect ARESTA), or directly fuse with other LEs (direct ARESTA) (Fig. 5, 6). These parallel pathways converge on Arl8b to drive endoggresome formation. We posit that the short but dynamic residence time of mutant PrP at the axonal cell surface tips the balance towards fusion by increasing the number of $\mathrm{PrP}^{\mathrm{PG} 14}$ vesicles inside axons available to Arl8b for fusion. In contrast, the steady presence of $\mathrm{PrP}^{\mathrm{WT}}$ at the plasma membrane (Fig. 2a) allows for recycling or clearance cycles that balance axonal influx and degradation resulting in an aggregatefree axon.

The identification of ARESTA highlights the discovery of a new type of intracellular aggregate compartment which we call the endoggresome. We define endoggresomes as structures composed of misfolded proteins that are formed inside endomembranes via the action of kinesin-dependent anterograde transport and requiring endosomal fusion, and that are not easily degraded. Previous observations of accumulations of misfolded proteins, including of $\mathrm{PrP}^{\mathrm{Sc}}$, into cytosolic aggresomes, IPODS, and/or JUNQ structures ${ }^{85,86}$ rendered our discovery somewhat unexpected. $\mathrm{PrP}^{\mathrm{Sc}}$ aggresomes were previously observed to form following proteasome inhibition and after retrotranslocation to the cytosol, where they are targeted for clearance by ERAD ${ }^{37}$. However, extensive characterization of the trafficking and turnover of many misfolded GPI-anchored PrP mutants showed that they are normally poor ERAD substrates, are 
not dependent on proteasomal degradation, and exit the ER for degradation in lysosomes $^{87}$. Thus, it is possible that endoggresome formation in axons is favored by misfolded PrP at steady state, but this may shift towards aggresome formation in the soma when and if RESET is overwhelmed or compromised during ER stress.

Several features distinguish endoggresomes from previously described aggresomes, IPODs or JUNQs ${ }^{85}$. First, the latter three are membrane-less structures while endoggresomes are formed inside endomembranes. Second, cytosolic aggregates depend on dynein-mediated retrograde movement to form at juxtanuclear or perivacuolar sites, but endoggresomes depends on kinesin-based anterograde transport to form peripherally, as removal of kinesin- 1 subunits dramatically inhibited their formation (Fig. 3). Third, the cores of cytosolic aggresomes, IPODs, and JUNQs are targeted for clearance by autophagosomes and/or proteasomal degradation, but our data show that endoggresomes are not easily targeted for degradation, as shown by the lack of lysosomal clearance that leads to their prolonged presence in axons. Finally, ample evidence suggests that the formation of cytosolic aggregates is a cytoprotective response mechanism to sequester proteins with chaperones to restore their native conformation, or if this fails, to rapidly destroy misfolded protein aggregates ${ }^{8}$. In contrast, our data show that endoggresomes impart toxicity by impairing the ability of neurons to regulate calcium intake dynamics, accelerating neuronal death.

The generality of endoggresomes and ARESTA within the prionopathies and to other neurodegenerative pathologies requires further investigation. However, our analysis indicates that at least in the case of $\operatorname{PrP}^{\mathrm{D} 178 \mathrm{~N} / \mathrm{M} 129}$, the formation of intra-axonal aggregates also depends on KIF5C (Supplementary Fig. 3), suggesting that ARESTA might be a common aggregation mechanism of familial PrP mutations. Given the reliance of tau, APP/A $\beta, \alpha$-synuclein, and huntingtin on endosomal pathways for their processing and spread, it is possible that ARESTA may also be implicated in the 
formation of misfolded aggregates of these proteins ${ }^{88-90}$. As genetic modulation of ARESTA components resulted in changes in endoggresome densities with measurable functional consequences to the heath and viability of neurons, the identification of the Arl8b/kinesin-1/HOPS ARESTA pathway and of endoggresomes provides an appealing anti-aggregation target that can modulate neuronal dysfunction and delineate the differential vulnerability of axons to aggregate-induced pathologies.

\section{METHODS}

\section{Mouse Lines and Maintenance}

Our mouse protocols were reviewed and approved by Institutional Animal Care and Use Committee (IACUC) at The Scripps Research Institute (Scripps Research) and all colonies were maintained following the guidelines recommended by the Department of Animal Resources (DAR) at Scripps Research. C57BL/6 wild-type mice were obtained from Charles River Laboratories. Kinesin-1C null $\left(\mathrm{Kif5}^{-\mathrm{l}^{-}}\right)$and $\mathrm{KLC} 1$ null $\left(\mathrm{Klc}^{\mathrm{H}^{-/}}\right)$mice were generated in Larry Goldstein's lab ${ }^{50} . \mathrm{Kif5}^{-/-}$mice were maintained as homozygotes and were backcrossed in the C57BL/6 background for at least 6 generations. Klc $1^{-/-}$mice were bred as heterozygotes to generate homozygous pups. The conditional homozygous kinesin-1B floxed mouse (Kif5 $\left.b^{\text {pflox/pflox }}\right)$ was generated by Nancy Jenkins and JianDong Huang ${ }^{72}$.

\section{Primary Neuronal Culture}

Primary hippocampal neuron cultures were prepared as described previously ${ }^{50}$. Briefly, hippocampi were dissected from 0 to 2-day-old mouse neonates in cold Hank's buffer solution (HBSS) (Gibco) supplemented with $0.08 \%$ D-glucose (Sigma), 0.17\% HEPES, and 1\% Pen-Strep (Gibco), filter sterilized and adjusted to $\mathrm{pH}$ 7.3. Dissected hippocampi were washed twice with cold HBSS (Gibco) and individually incubated at $37^{\circ} \mathrm{C}$ for $15-20$ 
minutes in a sterile solution of $45 \mathrm{U}$ papain (Worthington), $0.01 \%$ DNase (Sigma), $1 \mathrm{mg}$ DL-cysteine (Sigma), $1 \mathrm{mg} \mathrm{BSA} \mathrm{(Sigma)} \mathrm{and} 25 \mathrm{mg}$ D-Glucose (Sigma) in PBS (Gibco). Hippocampi were washed twice with Dulbecco's Modified Eagle Medium (DMEM) (Gibco) supplemented with $10 \%$ fetal bovine serum (FBS) (Gibco) (pre-heated to $37^{\circ} \mathrm{C}$ ) and disrupted by ten to twelve cycles of aspiration through a micropipette tip. Dissociated neurons were then resuspended in warm DMEM (Gibco) + 10\% FBS (Gibco) and plated in 24-well plates containing 12-mm glass coverslips pretreated with $50 \mu \mathrm{g} / \mathrm{ml}$ poly-L-lysine (Sigma) in Borate buffer (1.24 g boric acid (Fisher), $1.90 \mathrm{~g}$ borax (Sigma), in $500 \mathrm{~mL}$ cell-culture grade water, adjusted to $\mathrm{pH}$ 8.5, filtered sterilized). After 1 hour, media was replaced with Neurobasal-A medium (NBA) (Gibco), supplemented with 2\% B-27 (Gibco) and 0.25\% GlutaMAX (Gibco). Primary neurons were maintained in an incubator at $37^{\circ} \mathrm{C}$ and in a $5.5 \% \mathrm{CO}_{2}$ atmosphere.

For quantitative live imaging of axonal transport, hippocampal neurons were plated in microfluidic chambers, or in regular 24-well glass coverslips as described previously ${ }^{50}$. For calcium imaging, hippocampal neurons were plated directly onto \#1.5 glass-bottomed dishes (MatTek) precoated with $50 \mu \mathrm{g} / \mathrm{ml}$ poly-L-lysine (Sigma) in Borate buffer.

\section{Cell Culture}

Neuro2a (N2a) cells (ATCC) were grown in DMEM (Gibco), supplemented with $10 \%$ FBS (Gibco) and $1 \%$ Pen-Strep (Gibco) in an incubator at $37^{\circ} \mathrm{C}$ and in a $5.5 \% \mathrm{CO}_{2}$ atmosphere. Cells were passaged twice a week to low confluency using $0.05 \%$ TrypsinEDTA (Gibco).

\section{Design of DNA constructs}

The MoPrP.Xho PrP ${ }^{W T}$-EGFP and MoPrP.Xho PrP ${ }^{P G 14}$-EGFP constructs were a gift from 
David Harris (Boston University) ${ }^{36,60}$. All other constructs were generated using the InFusion ${ }^{\circledR}$ HD Cloning Plus Kit (Clontech) for the final cloning of DNA fragments into the recipient vector. All PCR amplifications were performed using the Phusion ${ }^{\circledR}$ High-Fidelity DNA Polymerase (NEB).

For the construction of MoPrP.Xho PrP ${ }^{W T}-m C h$ and MoPrP.Xho PrP ${ }^{P G 14}-m C h$ constructs, EGFP was replaced with mCh using assembly PCR. Briefly, three individual PCRs were performed to amplify the (1) N-terminus of $\mathrm{PrP}^{\mathrm{WT}}$ or $\mathrm{PrP}^{\mathrm{PG} 14}$, (2) C-terminus of $\operatorname{PrP},(3) \mathrm{mCh}$ including 20 and 22 bp overlaps to the 3' of (1) and 5' of (2), respectively.

(1) The N-terminus of PrP was amplified using primers

P1 N-Ter For: 5'-CTAGTGGTACCTCGAGATGGCGAACCTTGGCTAC-3'

P2 N-Ter Rev: 5'-CATGGTGGCGACCGGTGGATCC-3'

(2) The C-terminus of PrP was amplified using primers

P3 C-Ter For: 5'-TCCGGACTCAGATCTCGAGCTCAAG-3'

P4 C-Ter Rev: 5'-AGCAGGAAGGCTCGAGTCATCCCACGATCAGGAA-3'

(3) mCh was amplified using primers

P5 mCh For: 5'-ATCCACCGGTCGCCACCATGGTGAGCAAGGGCGAGGA-3'

P6 mCh Rev: 5'-GCTCGAGATCTGAGTCCGGACTTGTACAGCTCGTCCATGCCG-3'.

PCR products (1-3) were gel purified and subsequently stitched together (1 ng each), using a PCR reaction performed without primers. After 20 cycles, PCR was interrupted, primers $\mathrm{P} 1 \mathrm{For}$ and $\mathrm{P} 4 \mathrm{Rev}(0.5 \mu \mathrm{M}$ each) were added to specifically amplify the complete product. Reaction was supplemented with $100 \mu \mathrm{M}$ dNTP and $0.5 \mathrm{mM} \mathrm{MgCl}_{2}$ and ran for 30 more cycles. Generation of MoPrP.Xho PrP ${ }^{W T}$-mTagBFP2 and MoPrP.Xho PrP ${ }^{P G 14}-m$ TagBFP2 constructs was done using the same assembly PCR strategy.

P5 mTagBFP2 For: 5'-ATCCACCGGTCGCCACCATGGTGTCTAAGGGCGAAGA-3' 
P6 mTagBFP2 Rev: 5'-GCTCGAGATCTGAGTCCGGAATTAAGCTTGTGCCCCAGTT-3' Generation of the untagged MoPrP.Xho PrP ${ }^{W T}$ and MoPrP.Xho PrP ${ }^{\mathrm{PG} 14}$ was done using the same assembly PCR strategy.

(1) The N-terminus of PrP was amplified using primers

P1 N-Ter For: 5'-CTAGTGGTACCTCGAGATGGCGAACCTTGGCTAC-3'

P2 N-Ter Rev: 5'-CATGGTGGCGACCGGTGGATCC-3'

(2) The C-terminus of PrP was amplified using primers

P3 C-Ter For: 5'- CCGGTCGCCACCATGTCCGGACTCAGATCTCGAGCTCAAG -3'

P4 C-Ter Rev: 5'-AGCAGGAAGGCTCGAGTCATCCCACGATCAGGAA-3'

Generation of MoPrP.Xho PrP ${ }^{W T}-P A m C h$ and MoPrP.Xho PrP PG14-PAmCh was done using the same assembly PCR strategy from PAmCh1-C1 (a gift from Vladislav Verkhusha, Albert Einstein College of Medicine, New York) ${ }^{91}$, which contains the following mutations: E26V/A58T/K69N/L84F/N99K/S148L//165V/Q167P/L169V/I203R. P5 PAmCh For: 5'-ATCCACCGGTCGCCACCATGGTGAGCAAGGGCGAGGA-3' P6 PAmCh Rev: 5'-GCTCGAGATCTGAGTCCGGACTTGTACAGCTCGTCCATGCCG$3^{\prime}$

To generate Bungarotoxin binding sequence (BBS)-tagged constructs, MoPrP.Xho PrP ${ }^{W T}$-mCh-BBS and MoPrP.Xho PrP ${ }^{P G 14}$-mCh-BBS, a 13 amino-acid BBS tag (WRYYESSLEPYPD) was inserted at the BspEl site in the linker between mCh and C-terminal PrP sequence downstream of $\mathrm{mCh}$. The following oligos were used to amplify the BBS sequence:

BBS For: 5'-CCGGATGGAGATACTACGAGAGCTCCCTGGAGCCCTACCCTGACT-3' BBS Rev: 5'-TACCTCTATGATGCTCTCGAGGGACCTCGGGATGGGACTGAGGCC-3' The PCR products were annealed and cloned in $\mathrm{PrP}^{\mathrm{WT}}-\mathrm{mCh}$ in pcDNA3.1 plasmid and $\mathrm{PrP}^{\mathrm{PG} 14}-\mathrm{mCh}$ in pcDNA3.1 plasmid. PrP ${ }^{\mathrm{WT}}-\mathrm{mCh}-\mathrm{BBS}$ and $\mathrm{PrP}^{\mathrm{PG} 14}-\mathrm{mCh}-\mathrm{BBS}$ sequences were amplified by PCR and subcloned into the MoPrP.Xho vector. A similar strategy was 
used to generate the Thrombin cleavage sequence (TCS) tagged constructs MoPrP.Xho $\mathrm{PrP}^{\mathrm{WT}}$-mCh-TCS and MoPrP.Xho PrP ${ }^{\mathrm{PG} 14}$-mCh-TCS. The following oligos were used to amplify the 6 amino-acid TCS tag (LVPRGS), and to insert it at the BspEl site downstream of mCh:

TCS For: $5^{\prime}-$

GCTGTACAAGTCCGGACTGGTGCCGCGCGGCAGCTCCGGACTCAGATCTC-3’ TCS Rev: 5'-

\section{GAGATCTGAGTCCGGAGCTGCCGCGCGGCACCAGTCCGGACTTGTACAGC-3'}

To generate the LAMP1-EGFP construct, RFP was replaced with EGFP in the plasmid LAMP1-RFP (a gift from Erika Holzbaur, University of Pennsylvania) ${ }^{92}$. To generate ss(NPY)-EGFP, the first 84 nucleotides of Neuropeptide $Y$ were amplified from mouse brain cDNA and cloned between BgllI and BamHI sites in pEGFP-N3 vector. To design EGFP-Rab5, EGFP-Rab7 and EGFP-Rab11a, the corresponding sequences were amplified from mouse brain cDNA and cloned between Xhol and BamHI sites in the pEGFP-C1 vector (a gift from Hilal Lashuel, Swiss Federal Institute of Technology) ${ }^{93}$ : Rab5 For: 5'-TCTCGAGCTCAAGCTTTAATGGCTAATCGAGGAGCAACA-3' Rab5 Rev: 5'-TAGATCCGGTGGATCCTCAGTTACTACAACACTGGCTTCTGG-3' Rab7 For: 5’-TCTCGAGCTCAAGCTTATGACCTCTAGGAAGAAAGTGTTG-3' Rab7 Rev: 5'-TAGATCCGGTGGATCCTCAACAACTGCAGCTTTCTG-3' Rab11a For: 5'-TCTCGAGCTCAAGCTTATGGGAACACGCGACGACGTA-3' Rab11a Rev: 5'-TAGATCCGGTGGATCCGATGTTCTGACAGCACTGCACCTTT-3'. T34N and Q75L mutations were introduced in pDEST47-Arl8b ${ }^{\mathrm{WT}}$-GFP plasmid (Addgene \#67404), using the QuikChange II XL Site-Directed Mutagenesis kit (Agilent). To generate untagged Arl8b constructs, the Arl8b coding sequence was cloned in the pBICMV3 bidirectional promoter vector (Clontech). The KLC1-TAP plasmid was a gift from Larry S. B. Goldstein (University of California San Diego). 2xmCh-KIF5C was designed 
by subcloning 2xmCh from $\mathrm{KIF5C(1-560)-2xmChEF(C)} \mathrm{(Addgene} \mathrm{\# 61664)} \mathrm{in}$ pCDNA3.1. Then, full length KIF5C sequence was amplified from pGFP-KIF5C (a gift from Michelle Peckham, University of Leeds) ${ }^{94}$, and cloned downstream of 2xmCh. To generate the SKIP 1-186 construct, the sequence of the first 186 amino acids was cloned in pcDNA3.1 at the HindIII and BamHI site.

SKIP 1- 186 For: 5' - CTAGCGTTTAAACTTAAGCTTATGGAGCCGGGGGAGGTGAAG $-3^{\prime}$

SKIP 1 -186 Rev: 5' CCACACTGGACTAGTGGATCCGACCGAGCTGGGAAGGCGGTC - 3'

\section{Transfection}

Hippocampal neurons were transiently transfected using Lipofectamine 2000 (Thermo Fisher), following the manufacturer instructions. DNA and Lipofectamine were diluted in non-supplemented Neurobasal-A medium (Gibco). For most experiments, neurons grown on $12 \mathrm{~mm}$ coverslips in 24-well plates were transfected using $2 \mu \mathrm{L}$ of Lipofectamine and 0.4 or $0.8 \mu \mathrm{g}$ of DNA per well. Otherwise, neurons were grown in microfluidic chambers and transfected using $1.2 \mu \mathrm{L}$ of Lipofectamine and $0.5 \mu \mathrm{g}$ of DNA per chamber. Medium was changed 1 hour after transfection.

Neuro 2a cells were transiently transfected using Lipofectamine 2000 (Thermo Fisher) following the manufacturer instructions. DNA and Lipofectamine were diluted in DMEM (Gibco). Experiments were performed in 6 well plates, using $8 \mu \mathrm{L}$ of Lipofectamine and $2 \mu \mathrm{g}$ of DNA per well.

\section{Adenovirus transduction}

Cre-recombinase adenovirus transduction (University of lowa, Ad5CMVCre) was performed as described previously ${ }^{50}$. Briefly, hippocampal neurons isolated from 
Kinesin-1B conditional knockout mice $\left(\right.$ KifB $\left.^{\text {pflox/pflox }}\right)$ were treated at 6 DIV with 0,100 , or $400 \mathrm{MOI}$ of adenovirus, corresponding to $0,5 \times 10^{6}$, and $2 \times 10^{7}$ plaque-forming units (PFU), respectively. Neurons were incubated for 2 hours with the virus and then washed twice with Neurobasal-A medium (Gibco) containing 2\% B-27 (Gibco), and 0.25\% GlutaMAX (Gibco). Neurons were transfected with fluorescently-labeled PrP constructs at 9 DIV and imaged at 11 DIV.

\section{Adeno-associated virus transduction}

$\mathrm{PrP}^{\mathrm{WT}}-\mathrm{mCh}$ and $\mathrm{PrP}^{\mathrm{PG} 14}-\mathrm{mCh}$ were cloned into the pAAV-hSyn-MCS backbone between the BamHI and EcoRI sites (gift from T. Golde, University of Florida).

For: 5' - CCGCGAGCTCGGATCCATGGCGAACCTTGGCTACTG - 3’

Rev: 5' - CTTCCTGATCGTGGGATGAGAATTCCTCGAGCAGC - 3'

AAV virus was made using the AAV-DJ Helper Free Packaging System (Cell Biolabs) using published protocols ${ }^{95}$. Hippocampal neurons growing in 24-well plates were treated overnight with $4 \mu \mathrm{l}$ of the viral stock at DIV7 and imaged at DIV12.

\section{shRNAs \& siRNAs}

To knockdown Arl8b from neurons, the following 21-mer shRNA inserts were cloned separately in the pLKO.3G vector (Addgene plasmid \#14748): scrambled shRNA (CCTAAGGTTAAGTCGCCCTCG), Arl8b shRNA \#1 (CCTCTCGAAATGAACTGCATA), Arl8b shRNA \#3 (CGAGGAGTCAATGCAATTGTT). Dynein was knocked down by transfection of neurons with a previously validated DHC1 lentiviral construct (GTGATGCCATACGAGAGAA) and the scrambled construct (GCACACGTATCGACGTATC) $)^{50}$. Validation of shRNA constructs for reduction of Arl8b was done by transfecting mouse N2a cells at $40 \%$ confluency with pLKO3.G-Arl8b shRNA \#1 and \#2 either separately or together using Lipofectamine 2000 (Invitrogen). 
Transfection rates were $>80 \%$ and were ascertained by counts of GFP fluorescence on cell bodies. ShRNAs were expressed for 48 or $72 \mathrm{~h}$ prior to harvesting, lysed in radioimmunoprecipitation assay (RIPA) buffer and evaluated by Western-blot using rabbit anti-Arl8b (Proteintech) and anti-alpha-tubulin as a loading control (Sigma Aldrich). N2a cells were harvested at the same time points for reverse-transcriptase (RT) PCR generation using iScript cDNA Synthesis kit (Bio-Rad), and quantitative PCR (QPCR) was performed using the FastStart Universal SYBR Green Mastermix (Roche) to test for reduced Arl8b mRNA levels.

To knockdown VPS41 from neurons we used the ON-TARGET plus SMART pool siRNA from Dharmacon. VPS41 siRNA \#1: CCAAAGGAACAUUAAACGA, VPS41 siRNA \#2: GUUUGUACUGGCGGGAAA, VPS41 siRNA \#3: GGAGAAGAAUUUCACGAGA and VPS41 siRNA \#4: UGACAUAAGUCUUCGCCCA. Non-targeting siRNA \#1: UGGUUUACAUGUCGACUAA, was used as a control.

\section{Live-imaging microscopy}

Live imaging was done in soma and axons of hippocampal neurons at 9-15 DIV, usually two days after transfection, except otherwise stated. In some experiments, fluorescently labeled PrP constructs were co-transfected with soluble EGFP or mCh constructs in order to visualize the neuronal morphology and facilitate the determination of axonal polarity. Coverslips (12 mm), were transferred and flipped onto $35 \mathrm{~mm}$ glass bottom dishes (MatTek) containing $2 \mathrm{~mL}$ of Neurobasal-A medium (Gibco,) containing 2\% B-27 (Gibco) and $0.25 \%$ GlutaMAX (Gibco) media. Neurons were imaged using a Nikon Ti-E Perfect Focus inverted microscope equipped with a total internal reflection fluorescence microscopy (TIRFM) setup, with an Andor iXon + DU897 EM Camera, and a 100X/1.49 NA oil objective. A $488 \mathrm{~nm}$ laser was used for detecting GFP and a $561 \mathrm{~nm}$ laser to detect $\mathrm{mCh}$. Lasers were positioned at varying angles for pseudo-TIRFM acquisition. 
Transfection rates were $\sim 1 \%$, which enabled imaging of individual neurons.

Time-lapse movies of axonal transport had different duration and framerates depending on the type of cargo analyzed. Movies of fast vesicular transport were $15 \mathrm{sec}$ long and collected at a frame rate of 10 frames/sec $(10 \mathrm{~Hz})$. Movies of aggregate transport were 5 min $(300 \mathrm{sec})$ long and collected at a frame rate of 1 frame/sec $(1 \mathrm{~Hz})$. Movies of LAMP1-EGFP vesicular transport were $1 \mathrm{~min}$ long and collected at a frame rate of 5 frame/sec $(5 \mathrm{~Hz})$. For all images and movies acquired, exposure time was set to $100 \mathrm{~ms}$. Pixel size was $0.16 \mu \mathrm{m}$. Plates with cultured neurons were maintained at $37^{\circ} \mathrm{C}$ and $5.5 \% \mathrm{CO}_{2}$ throughout the total imaging period. All axonal transport movies were taken in a central region of the axon, at least $150 \mu \mathrm{m}$ away from both the soma and the axon tip.

For green/red axonal cotransport analyses, we performed near-simultaneous two-color imaging. Exposure times were set to $75 \mathrm{~ms}$ and red and green images were collected with a $50 \mathrm{~ms}$ delay between them using Nikon's proprietary LAMBDA 10-3 optical filter switch. For axonal transport live imaging, high-resolution imaging of vesicle cotransport was done using a high frame rate: 30 seconds long and at 5 frames/second (5 Hz). Low frame rate cotransport movies were obtained for analyses of aggregate transport: 5 min (300 seconds) long and collected at 1 frame/sec $(1 \mathrm{~Hz})$. Time-lapse imaging was performed during $5 \mathrm{~min}$ at a frame rate of 5 frames per sec.

\section{Fixation and Immunofluorescence}

Neurons were fixed with 4\% paraformaldehyde (PFA, Electron Microscopy Services) containing $4 \%$ sucrose (Sigma) for $30 \mathrm{~min}$ at $37^{\circ} \mathrm{C}$. Cells were washed once in $50 \mathrm{mM}$ Glycine in PBS and 3 more times in PBS. If applicable, cells were permeabilized by incubating the coverslips in $0.1 \%$ Triton X-100 (Sigma) in PBS for 8-10 minutes followed by 3 washes with PBS. Coverslips were incubated in blocking solution (10\% IgG-Free BSA, $5 \%$ donkey serum or goat serum in PBS, Jackson ImmunoResearch) for 30 
minutes at room temperature (RT). Primary antibodies were incubated in blocking solution for 1 hour at RT or overnight at $4^{\circ} \mathrm{C}$. After 3 washes in PBS, secondary antibodies were incubated in blocking solution for 1 hour at RT or overnight at $4^{\circ} \mathrm{C}$. If applicable, neurons were counterstained with 300 nM DAPI (Thermo Fischer) for 5 minutes. After 3 washes in PBS, coverslips were washed once in $\mathrm{H}_{2} \mathrm{O}$ and mounted in ProLong Diamond antifade reagent (Thermo Fisher).

The following antibodies were used for immunofluorescence (IF): mouse antiKDEL (1:100, Santa Cruz Biotechnology); rabbit anti-GM130 (1:100, Abcam); goat antimouse Cathepsin B (1:40, R and D systems); rabbit polyclonal IgG anti-mCh (1:100 Gentex), recombinant Fab anti-PrP (1:200 clone HuM-D13) ${ }^{96}$, rabbit anti-Arl8b (1:200, ProteinTech), and mouse anti-Vps41 (1:100 clone D-12, Santa Cruz Biotechnology). The following antibodies were used for Western blot (WB): rabbit anti-Arl8b (1:200,

ProteinTech) and mouse anti-alpha-tubulin (1:500, Sigma).

\section{Correlative Fluorescence and Scanning Electron Microscopy (SEM)}

Hippocampal neurons were plated in microfluidic chambers and co-transfected at 8 DIV with $\mathrm{PrP}^{\mathrm{PG} 14}$-mCh and soluble EGFP. Two days after transfection, the neurons inside entire microfluidic chamber were imaged using live-fluorescence microscopy (Nikon S Plan Fluor ELWD 20X/0.45 [infinity]/0-2 Objective) to map the location of transfected neurons. Shortly thereafter, neurons were initially fixed in ice-cold $2.5 \%$ glutaraldehyde in $0.1 \mathrm{M} \mathrm{Na}$ cacodylate buffer $(\mathrm{pH} 7.3)$ with the addition of $1 \%$ trehalose during which the mold was carefully removed to expose fully the cultured cells. After a buffer wash the cells were fixed in $1 \%$ osmium tetroxide, washed thoroughly with distilled water and dehydrated in graded ethanol series. Following the $100 \%$ ethanol dehydration, the samples are immersed in 100\% hexamethyldisilazane (HMDS) for 3 minutes and allowed to dry. Each coverslip was mounted on a stub with carbon tape and samples 
were sputter coated with iridium at $10 \mu \mathrm{A}$ to a thickness of approximately $5 \mathrm{~nm}$ (EMS model 150T S). The coverslips were then examined on a Hitachi S4300 SEM (Hitachi High Technologies America Inc., Pleasanton CA) at 5kV with settings adjusted according to needs.

\section{Correlative Fluorescence and Serial Sectioning Scanning Fluorescence Electron Microscopy (S3EM)}

Hippocampal neurons were plated on $35-\mathrm{mm}$ gridded \#1.5 glass bottom dish (Cellvis) pretreated with $50 \mu \mathrm{g} / \mathrm{ml}$ poly-L-lysine (Sigma) in Borate buffer and transfected at $8 \mathrm{DIV}$ with PrP ${ }^{P G 14}$-mCh using Lipofectamine 2000 (Thermo Fisher). Two days after transfection, neurons were first imaged using live-fluorescence and phase-contrast microscopy (Nikon S Plan Fluor ELWD 20X/0.45 [infinity]/0-2 Objective) to map the location of transfected neurons on the grid (Supplementary Fig. 3e). Then PrP ${ }^{P G 14}-m C h$ aggregate sites were imaged at high resolution using super-resolution radial fluctuation (SRRF) microscopy ${ }^{97}$ (20 frames/second, 100 frames) with a pseudo-total internal reflection fluorescence microscopy (pTIRFM) setup, with an Andor iXon + DU897 EM Camera, and a 100X/1.49 NA oil objective. Samples were then fixed on the gridded culture dish while imaging on the microscope by carefully mixing an equal volume of $2 \mathrm{X}$ EM-fixative ( $5 \%$ glutaraldehyde, $4 \%$ paraformaldehyde in $0.1 \mathrm{M}$ cacodylate buffer with $3 \mathrm{mM} \mathrm{CaCl}_{2}$ ) warmed to $37^{\circ} \mathrm{C}$ with $2 \mathrm{~mL}$ of Neurobasal-A medium (Gibco,) containing $2 \%$ B-27 (Gibco) and 0.25\% GlutaMAX (Gibco). Samples were immediately removed from the microscope, fixative-media solution was discarded, and cells were left in fresh icecold $1 \mathrm{X}$ fixative for 90 minutes and rinsed three times with ice cold $0.1 \mathrm{M}$ cacodylate buffer with $3 \mathrm{mM} \mathrm{CaCl}_{2}$. Coverslips were post-fixed and stained with $1.5 \%$ reduced osmium for 35 minutes, rinsed five times with MilliQ water, and stained again with $1 \%$ 
aqueous uranyl acetate for 1 hour at room temperature, before serial dehydration with graded solutions of ice-cold ethanol in water. Samples were then fully dehydrated in two 10 minute rinses of anhydrous ethanol at room temperature before infiltration with Durcupan resin. After 3:1, 1:1, 1:3 ethanol-resin two-hour infiltration steps, samples were infiltrated with pure resin for two hours before another change of fresh pure resin and left overnight at room temperature. In the morning, a final change of fresh resin was performed, taking care to let the viscous resin fully drain by inverting the coverslip before adding the final aliquot of fresh resin from the edge of the dish. Resin was added to fill the dish up to $2 \mathrm{~mm}$ and infiltrated samples were polymerized for 48 hours at $65^{\circ} \mathrm{C}$.

After polymerization, coverslips were dissolved by immersion in concentrated hydrofluoric acid. Correlative light-electron microscopy (CLEM) was achieved using laser branded fiducials in a thinly embedded sample, as previously described ${ }^{98}$. Briefly, regions of interest (ROIs) that included SRRF-imaged $\mathrm{PrP}^{\mathrm{PG} 14}-\mathrm{mCh}$ axon segments were identified by their grid positions and dark osmium staining. ROls were marked using the cutting laser of a Zeiss PALM laser cutting microscope to provide orientation and fiducial guides for further trimming and ultramicrotomy. ROIs were carefully identified under a dissecting microscope and excised from the coverslip using a jeweler saw and a scalpel, with careful thought given to the future blockface orientation. The small $(1 \times 2 \times 2 \mathrm{~mm})$ sample block was glued to a blank resin block such that the ROls were orthogonal to the cutting plane and approximately $100 \mu \mathrm{m}$ from the cutting surface. Laser marks on the block face helped to identify the location of the ROI using the ultramicrotome optics. The block was carefully trimmed using a $90^{\circ}$ diamond trimming knife (Diatome) so that the block width bounded the fiducial laser marks, and the two sides of the block face were perfectly parallel for serial sectioning when turned $90^{\circ}$. 
The ROI was approached using the trimming knife to provide a perfectly smooth block face. When the fiducials marking the location of ROI became challenging to visualize

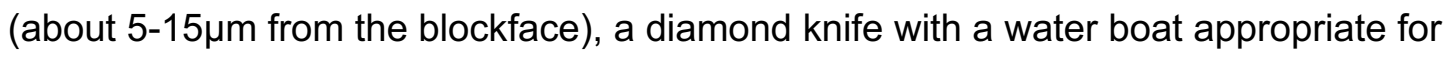
serial sectioning (Histo $45^{\circ}$, Diatome) was installed on the ultramicrotome and $50 \mathrm{~nm}$ serial sections were collected on a series of plasma cleaned custom-diced silicon chips (University Wafer, Boston, MA) immersed in the knife boat. About 200 serial sections were collected in total over four chips.

The chips were mounted on aluminum stubs using double-sided carbon sticky tape and loaded into a Zeiss Sigma VP scanning electron microscope (SEM). Chip mapping and the imaging of serial sections was facilitated by SmartSEM (Zeiss) and Atlas 5 (FIBICS) software packages. Maps of all of the serial sections on the silicon chips were collected at $500 \mathrm{~nm} / \mathrm{px}$. Laser marks could be identified in the resin boundary at low magnification, demarcating the ROI in each section. The ROI of the axon segment containing the $\mathrm{PrP}^{\mathrm{PG} 14}$-mCh aggregates was identified and captured across 40 serial section scanning electron micrographs. ROls were imaged at $2 \mathrm{~nm} / \mathrm{px}$ using an electron backscatter detector (Gatan). In high-vacuum mode, a $3 \mathrm{keV}$ beam in high-current mode with a 30 $\mu \mathrm{m}$ aperture at a working distance of $9 \mathrm{~mm}$ was found to produce signal from which we could resolve membrane and organelle (eg., microtubule) ultrastructure. Sections were aligned using Photoshop (Adobe) and elastic alignment functions ${ }^{99}$ embedded in Fiji $(\mathrm{NIH})$.

The orthogonal projection (eg., $\mathrm{x}-\mathrm{z}$ ) of the S3EM data was used to correlate the SRRFprocessed data and produce overlay (Fig. 3e and Supplementary Fig. 3e). Sections were sampled from throughout the aggregate and non-aggregate sites with reference to the correlated volume. 


\section{Hippocampal Neuron Live Assays with Various Drug Treatments and Fluorescent}

\section{Dyes}

To retain PrP ${ }^{P G 14}$ in the ER, Brefeldin-A (BFA) (BioLegend) was applied at $5 \mu \mathrm{g} / \mathrm{mL}$ at the same time as $\mathrm{PrP}^{\mathrm{WT}}-\mathrm{mCh}$ or $\mathrm{PrP}^{\mathrm{PG} 14}-\mathrm{mCh}$ transfections and incubated for 2-6 hours. Neurons are washed 2-3 times with Neurobasal-A medium (Gibco) prior to fixation.

For characterization of $\mathrm{PrP}^{\mathrm{PG} 14}$ at the cell surface of the soma or axons, neurons grown in 24-well plates, were transfected with a $\mathrm{PrP}^{\mathrm{PG} 14}-\mathrm{mCh}-\mathrm{BBS}$ construct for 1 hour. Neurons were treated with AlexaFluor647-labeled-a-Bungarotoxin (BTX-a647, Molecular Probes) by applying it to the media and as described previously ${ }^{66}$. Briefly, we pretreated neurons for 1 hour with $100 \mu \mathrm{M}$ tubocurarine hydrochloride (Sigma), to eliminate nonspecific binding to other receptors ${ }^{66}$. Neurons were then incubated with $7 \mu \mathrm{g} / \mathrm{mL}$ BTXa647 in the media, during indicated times and at indicated temperatures. To inhibit clathrin mediated-endocytosis, $80 \mu \mathrm{M}$ Dynasore (Sigma) was added 30 minutes prior to BTX-a647 treatment and kept during the labeling. DMSO was used as a control. Internalized $\mathrm{PrP}^{\mathrm{PG} 14}$ particles were defined as $\mathrm{PrP}^{\mathrm{PG} 14}$-mCh-BBS particles that were both positively labeled with BTX-a647 and that were actively transporting within axons. Quantification of internalization in the presence or absence of Dynasore was performed by measuring the proportion of BTX-a647 positive particles that were actively transporting per movie.

$\mathrm{PrP}^{\mathrm{PG} 14}$ surface expression pattern in axons was analyzed by incubating neurons with BTX-a647 during 10 minutes at $4^{\circ} \mathrm{C}$ before fixation. LysoTracker Green (Molecular Probes) was incubated at $50 \mathrm{nM}, 1-2$ hours before live-imaging. Magic Red from the Cathepsin B Assay (ImmunoChemistry Technologies), was incubated 1-2 hours before live-imaging. 260X DMSO stock of Magic Red dye was diluted in sterile $\mathrm{diH}_{2} \mathrm{O}$ and $10 \mu \mathrm{L}$ was applied onto neurons in each well containing $250 \mu \mathrm{l}$ of Neurobasal-A medium (Gibco). Bafilomycin A1 (Sigma) was incubated at $10 \mathrm{nM}$ during 6hours. Longer 
incubation times affected neuronal survival. DMSO was used as a control.

For characterization of $\mathrm{PrP}^{\mathrm{PG} 14}$ cleavage at the cell surface, hippocampal neurons were transfected with $\mathrm{PrP}^{\mathrm{PG} 14}-\mathrm{mCh}-\mathrm{TCS}$ or $\mathrm{PrP}^{\mathrm{PG} 14}-\mathrm{mCh}$. Neurons were incubated with or without 5 units/ml of Thrombin protease (Sigma) 2 hours after transfection. This treatment was maintained for 48 hours. We did not observe any effect of 5 units/ml Thrombin treatment on neuronal survival.

For characterization of (in)dependence between direct and indirect ARESTA, hippocampal neurons were transfected with $\mathrm{PrP}^{\mathrm{PG} 14}-\mathrm{mCh}-\mathrm{TCS}$ and Arl8b $\mathrm{b}^{\mathrm{WT}}-\mathrm{GFP}$. Neurons were incubated with or without 5 units/ml of Thrombin protease (Sigma) 24 hours after transfection. This treatment was maintained for 24 hours. We did not observe any effect of 5 units/ml Thrombin treatment on neuronal survival.

\section{Photoactivation of PrP ${ }^{\text {PG14 }}$-PAmCh}

Neurons were cotransfected with EGFP-Rab7 and PrPPG14-PAmCh. Two days after transfection, PrP ${ }^{\mathrm{PG} 14}$-PAmCh was photoactivated using a $405 \mathrm{~nm}$ laser burst pointed at the cell body (40\% laser power, 3 sec exposure), and neurons were imaged using livefluorescence microscopy. The fate of the photoactivated molecules and their delivery into axons was followed for 60 minutes, by taking $5 \times 5$ stitching images every 5 minutes.

\section{Proteinase-K Resistance Assay}

N2a cells were transfected with $\mathrm{PrP}^{\mathrm{WT}}$-mCh or $\mathrm{PrP}^{\mathrm{PG} 14}-\mathrm{mCh}$ (pcDNA3.1 vector), and lysed 48 hours after transfection in PBS containing 0.5\% Nonidet P-40 (Sigma), 0.5\% sodium deoxycholate (Sigma), 0.2\% Sarkosyl (Sigma), and 0.5\% Triton X-100. Proteins in $500 \mu \mathrm{g}$ of lysate were digested using $0,0.05,0.1,0.5,1$ or $5 \mu \mathrm{g} / \mathrm{ml}$ of Proteinase $\mathrm{K}$ (PK) (Thermo Fisher), during 30 minutes at $37^{\circ} \mathrm{C}$. Reaction was blocked by adding 0.8 $\mathrm{mg} / \mathrm{ml}$ of freshly prepared PMSF (Sigma). The equivalent of $20 \mu \mathrm{g}$ of each sample was 
analyzed by immunoblotting using an anti-PrP specific antibody (D13) (gift of D. Burton, Scripps Research). Samples corresponding to 1 and $5 \mu \mathrm{g} / \mathrm{ml}$ of PK were also precipitated using trichloroacetic acid (TCA) (Sigma) to concentrate the proteins. The equivalent of $120 \mu \mathrm{g}$ of each sample was analyzed by immunoblotting.

\section{SDS-Page and Immunoblotting}

Neuronal lysates were resuspended in Laemmli buffer and resolved by SDS-PAGE in 8$12 \%$ polyacrylamide gels. Proteins were electrotransferred onto PVDF membranes. Membranes were blocked for 30 minutes at RT in TBS containing $0.1 \%$ Tween 20 and $5 \%$ dry milk. They were incubated overnight at $4{ }^{\circ} \mathrm{C}$ with primary antibodies diluted in TBS-Tween-milk. After six washes in TBS-Tween, the membranes were further incubated for $1 \mathrm{~h}$ at RT with fluorescent secondary antibodies (Li-Cor) diluted in TBSTween-milk. After 6 more washes in TBS-Tween, the membranes were imaged using LiCor Odissey imaging system using recommended antibodies.

\section{Calcium Imaging}

Hippocampal cultures from WT (C57BL6) or Kif5c $\mathrm{c}^{-/}$mice were plated directly on \#1.5 glass-bottomed dishes (MatTek), at the density of 8-15 neurons per $420 \mu^{2}$ field of view. Cultures were loaded with $2 \mu \mathrm{M}$ Fluo-4 AM (Thermo Fisher) in Neurobasal-A media supplemented with $\mathrm{B} 27$ without phenol red for 30 minutes at $37^{\circ} \mathrm{C}$ in a $5.5 \% \mathrm{CO}_{2}$ atmosphere, as recommended by Invitrogen protocol. Cultures were washed 3 times and incubated for another 30 minutes in the B-27 supplemented Neurobasal-A without phenol red prior to imaging to allow complete de-esterification of intracellular AM esters. Prior and during imaging, calcium imaging buffer (140 mM NaCl, $5 \mathrm{mM} \mathrm{KCl,} 2 \mathrm{mM}$ $\mathrm{CaCl}_{2}, 0.8 \mathrm{mM} \mathrm{MgCl}, 10 \mathrm{mM}$ glucose, $10 \mathrm{mM}$ HEPES [pH 7.4]) was applied. 
Neurons were imaged using a Nikon S Plan Fluor ELWD 20X/0.45 [infinity]/0-2 Objective in a temperature-controlled enclosure set to $37^{\circ} \mathrm{C}$. Epifluorescence excitation was provided by a metal-halide-doped mercury arc lamp (Nikon Intensilight). High temporal-resolution images ( 5 frames/second) were taken for 1 minute with EM-CCD camera (Andor iXon3 897). Fluo-4 AM fluorescence were collected using Chroma filters (ET-GFP [ET470/40x T495lpxr, ET525/50m]. Depolarization was induced by applying $\mathrm{KCl}$ to a final concentration of $30 \mathrm{mM}$ at the 30 -second time point during acquisition. Still images of $\mathrm{PrP}^{\mathrm{WT}}$-mCh or $\mathrm{PrP}^{\mathrm{PG} 14}$-mCh of the same region were collected using ETDsRed [ET545/30x, T570Ip, ET620/60m] Chroma filter.

\section{Cell Death Assay and Imaging}

ReadyProbes ${ }^{\circledR}$ Cell Viability Imaging Kit (Blue/Green) (Thermo Fisher) was used to quantify cell death. In brief, two drops of NucBlue ${ }^{\circledR}$ Live reagent (Hoechst 33342) and two drops of NucGreen® Dead reagent were added onto 10-14 DIV hippocampal neurons grown on the coverslips containing $1 \mathrm{ml}$ of Neurobasal-A medium (NBA) (Gibco), supplemented with 2\% B-27 (Gibco) and 0.25\% GlutaMAX (Gibco). Neurons were incubated at $37^{\circ} \mathrm{C}$ for 30 minutes prior to fixation with $4 \%$ PFA (Electron Microscopy Services) containing $4 \%$ sucrose (Sigma) for $30 \mathrm{~min}$ at $37^{\circ} \mathrm{C}$. Cells were washed once in 50 mM Glycine in PBS and 3 more times in PBS before mounted in ProLong Diamond antifade reagent (Thermo Fisher).

Fixed neurons were imaged using a Nikon S Plan Fluor ELWD 20X/0.45 [infinity]/0-2 Objective. Epifluorescence excitation was provided by a metal-halide-doped mercury arc lamp (Nikon Intensilight). Blue and Green nuclei staining were collected using UV-2E/C [AT350/50x, T400Ip, ET460/50m] and ET-GFP [ET470/40x, T495Ipxr, ET525/50m] Chroma filters respectively. $\mathrm{PrP}^{\mathrm{WT}}$-mCh or $\mathrm{PrP}^{\mathrm{PG} 14}-\mathrm{mCh}$ fluorescence were collected using ET-DsRed [ET545/30x, T570Ip, ET620/60m] Chroma filter. 


\section{QUANTIFICATION AND STATISTICAL ANALYSIS}

\section{Neuronal Soma Image Analysis}

All images of neuronal soma were processed in ImageJ (National Institutes of Health)

100. Rolling-ball background fluorescence correction was applied to all images. 3D

Deconvolution was applied using synthetic Gaussian point spread function (PSF) and

Richardson-Lucy (RL) algorithms in DeconvolutionLab2 ${ }^{101}$. All images and line scan

fluorescence intensity graphs were displayed as maximum intensity projection.

\section{Analysis of Axonal Transport Dynamics from Time-lapse Movies}

Axonal transport analysis was performed using the custom-made and previously validated KymoAnalyzer ImageJ package of macros ${ }^{70}$. KymoAnalyzer is freely available for download from our lab website (http://www.encalada.scripps.edu/kymoanalyzer), and a detailed description are available here and in ${ }^{70}$. Briefly, kymographs were generated from time-lapse movies. Particle trajectories were manually assigned from the kymograph images. Track and segment-related parameters were automatically calculated by KymoAnalyzer.

\section{Colocalization and Cotransport Quantifications}

We determined colocalization by merging line-scan intensity profiles of different channels in the same graph and quantifying the average number of peaks that overlapped between different channels. For cotransport analyses, individual and merged color kymographs were generated and quantification of the average number of tracks that overlapped between each pair of vesicular cargoes was analyzed. Vesicle tracks were sorted in anterograde, retrograde and stationary populations, or as indicated for 
individually analyzed parameters. Aggregate tracks were sorted in mobile and stationary populations, or as indicated for individually analyzed parameters.

\section{Quantification of Particle Densities in Axons}

$\mathrm{PrP}^{\mathrm{PG} 14}$ aggregate densities were quantitated from still images of axons transfected with fluorescently-labeled PrP constructs, as follows: fluorescence background was subtracted by quantification of the maximal gray intensity of five small $\mathrm{PrP}^{\mathrm{PG} 14}$ vesicles (as define by visual comparison of size to those vesicles that actively move in live movies), quantified for each image analyzed. The calculated intensity average values were subtracted from each image using the Brightness and Contrast tool of ImageJ. The particles still present in the image after thresholding were considered as aggregates.

Axon length was measured using the polyline tool of ImageJ. Aggregate density was expressed as the average number of aggregates per $100 \mu \mathrm{m}$ of axon length.

For Lysotracker-positive vesicle densities, we measured the axon length using the polyline tool of ImageJ and quantified all the particles labelled by Lysotracker-Green along this line. Densities were plotted per $100 \mu \mathrm{m}$ of axon length.

\section{ERSE-mCh and Vps41 Fluorescence Quantification}

Neurons were cotransfected with the pEGFP-C1, moPrP.Xho PrPWT-EGFP or moPrP.Xho PrPPG14-EGFP and the transcriptional ER stress reporter ERSE-mCh (a gift from Larse Plate, Vanderbilt University) ${ }^{64}$ Increases in the activity of ER stress pathways activate ERSE and soluble mCh is synthesized.

We quantified total fluorescence intensity (gray values, arbitrary unit, a.u.) of ERSE-mCh and Vps41 using ImageJ, using circular ROIs of $30 \mu \mathrm{m}$ in diameter centered around the soma. For axonal total fluorescence intensity, polyline tool was used to draw ROls that cover the entire axonal length. 


\section{Calcium Imaging Analysis}

Regions of interest (ROls) were marked around soma of neurons expressing $\mathrm{PrP}^{\mathrm{WT}}-\mathrm{mCh}$ or $\mathrm{PrP}^{\mathrm{PG} 14}-\mathrm{mCh}$, and we measured average fluorescence for each $\mathrm{ROI}$ at a given timepoint. The average Fluo-4 AM fluorescence overtime was collected using an ImageJ Plugin Time Series Analyzer V3 and normalized to $\Delta \mathrm{F} / \mathrm{F}$ by subtracting fluorescence intensity of each time point $(\mathrm{F})$ to an average fluorescence prior to addition of $\mathrm{KCl}\left(\mathrm{F}_{0}\right)$ and divided by F. Rolling-ball background fluorescence correction was applied to all images prior to fluorescence intensity analysis.

\section{Cell Death Quantitation}

All image processing was done in ImageJ. The total number of NucBlue ${ }^{\circledR}$ Live positive nuclei (blue channel) were determined as follows: background fluorescence was corrected on each image, by applying a Gaussian blur filter with a radius of 12 to a duplicate of each image and subtracting this filtered image from the original. An automatic threshold was applied to the resulting images using the Li algorithm followed by a watershed filter, both in ImageJ. Total nuclei were counted using a minimum size threshold of 15 and circularity of 0.10 . The total amount of NucGreen® Dead positive nuclei (green channel) were determined using the same method, now using a Gaussian blur filter with a radius of 10 , thresholding using the Yen algorithm available in ImageJ, and nuclei determination using a minimum size threshold of 15 and circularity of 0.10 .

\section{Generation of Graphs and Figures}

Graphs of average values were generated using Microsoft Excel. Cumulative frequency graphs were generated using the CDF function in MATLAB (Mathworks). Box plots were generated using BoxPlotR, an application in the shiny package from RStudio 
(http://shiny.chemgrid.org/boxplotr/). Figures were drawn in Adobe Illustrator. Neuron cartoon (graphical abstract) was downloaded from Biorender (https://biorender.com/).

\section{Statistical Analyses}

All the transport parameters measured in this study were first tested for normality using the MATLAB Lilliefors test. For parameters following a normal distribution, we performed a Student's t-test. For non-normal parameters, we performed a permutation t test (rndttest function in MATLAB). For all parameters, differences in medians were also checked using the Wilcoxon rank-sum test (rnk function in MATLAB). The nonparametric Kolmogorov-Smirnov test was used to evaluate the equality of two sample distributions (in cumulative distribution functions). Most of the parameters are presented as mean \pm SEM. Multiple comparison corrections were used where appropriate. Details about each parameter, including definition of center, value and definition of $n$, statistical test used and $p$ values, can be found in specific figures and/or in figure legends.

\section{REFERENCES}

1 Lim, J. \& Yue, Z. Neuronal aggregates: formation, clearance, and spreading. Dev Cell 32, 491-501, doi:10.1016/j.devcel.2015.02.002 (2015).

2 Vaquer-Alicea, J. \& Diamond, M. I. Propagation of Protein Aggregation in Neurodegenerative Diseases. Annu Rev Biochem 88, 785-810, doi:10.1146/annurev-biochem-061516-045049 (2019).

3 Maday, S., Twelvetrees, A. E., Moughamian, A. J. \& Holzbaur, E. L. Axonal transport: cargo-specific mechanisms of motility and regulation. Neuron 84, $292-$ 309, doi:10.1016/j.neuron.2014.10.019 (2014).

$4 \quad$ Farfel-Becker, T. et al. Neuronal Soma-Derived Degradative Lysosomes Are Continuously Delivered to Distal Axons to Maintain Local Degradation Capacity. Cell Rep 28, 51-64 e54, doi:10.1016/j.celrep.2019.06.013 (2019).

5 Cai, Q. et al. Snapin-regulated late endosomal transport is critical for efficient autophagy-lysosomal function in neurons. Neuron 68, 73-86, doi:10.1016/j.neuron.2010.09.022 (2010). 
6 Maday, S. Mechanisms of neuronal homeostasis: Autophagy in the axon. Brain Res 1649, 143-150, doi:10.1016/j.brainres.2016.03.047 (2016).

7 Overly, C. C. \& Hollenbeck, P. J. Dynamic organization of endocytic pathways in axons of cultured sympathetic neurons. J Neurosci 16, 6056-6064 (1996).

8 Kopito, R. R. Aggresomes, inclusion bodies and protein aggregation. Trends Cell Biol 10, 524-530, doi:10.1016/s0962-8924(00)01852-3 (2000).

9 Kaganovich, D., Kopito, R. \& Frydman, J. Misfolded proteins partition between two distinct quality control compartments. Nature 454, 1088-1095, doi:10.1038/nature07195 (2008).

10 Guthrie, C. R. \& Kraemer, B. C. Proteasome inhibition drives HDAC6-dependent recruitment of tau to aggresomes. J Mol Neurosci 45, 32-41, doi:10.1007/s12031-011-9502-x (2011).

11 Kumar, R., Nawroth, P. P. \& Tyedmers, J. Prion Aggregates Are Recruited to the Insoluble Protein Deposit (IPOD) via Myosin 2-Based Vesicular Transport. PLoS Genet 12, e1006324, doi:10.1371/journal.pgen.1006324 (2016).

12 Taylor, J. P. et al. Aggresomes protect cells by enhancing the degradation of toxic polyglutamine-containing protein. Hum Mol Genet 12, 749-757, doi:10.1093/hmg/ddg074 (2003).

13 Tanaka, M. et al. Aggresomes formed by alpha-synuclein and synphilin-1 are cytoprotective. J Biol Chem 279, 4625-4631, doi:10.1074/jbc.M310994200 (2004).

14 Farrawell, N. E. et al. Distinct partitioning of ALS associated TDP-43, FUS and SOD1 mutants into cellular inclusions. Scientific reports 5, 13416 (2015).

15 Olzmann, J. A., Li, L. \& Chin, L. S. Aggresome formation and neurodegenerative diseases: therapeutic implications. Current medicinal chemistry 15, 47-60 (2008).

16 Johnston, J. A., Ward, C. L. \& Kopito, R. R. Aggresomes: a cellular response to misfolded proteins. J Cell Biol 143, 1883-1898, doi:10.1083/jcb.143.7.1883 (1998).

17 Wang, C., Telpoukhovskaia, M. A., Bahr, B. A., Chen, X. \& Gan, L. Endolysosomal dysfunction: a converging mechanism in neurodegenerative diseases. Curr Opin Neurobiol 48, 52-58, doi:10.1016/j.conb.2017.09.005 (2018).

18 Stahl, N., Borchelt, D. R., Hsiao, K. \& Prusiner, S. B. Scrapie prion protein contains a phosphatidylinositol glycolipid. Cell 51, 229-240 (1987).

19 Budka, H. et al. Neuropathological diagnostic criteria for Creutzfeldt-Jakob disease (CJD) and other human spongiform encephalopathies (prion diseases). Brain Pathol 5, 459-466, doi:10.1111/j.1750-3639.1995.tb00625.x (1995). 
20 Prusiner, S. B. Biology and genetics of prions causing neurodegeneration. Annu Rev Genet 47, 601-623, doi:10.1146/annurev-genet-110711-155524 (2013).

21 Silveira, J. R., Caughey, B. \& Baron, G. S. Prion protein and the molecular features of transmissible spongiform encephalopathy agents. Curr Top Microbiol Immunol 284, 1-50 (2004).

22 Prusiner, S. B. Novel proteinaceous infectious particles cause scrapie. Science (New York, N Y) 216, 136-144 (1982).

23 Collinge, J. Prion diseases of humans and animals: their causes and molecular basis. Annual review of neuroscience 24, 519-550 (2001).

24 Kraus, A., Groveman, B. R. \& Caughey, B. Prions and the potential transmissibility of protein misfolding diseases. Annu Rev Microbiol 67, 543-564, doi:10.1146/annurev-micro-092412-155735 (2013).

25 Mastrianni, J. A. The genetics of prion diseases. Genet Med 12, 187-195, doi:10.1097/GIM.0b013e3181cd7374 (2010).

26 Watts, J. C. \& Prusiner, S. B. Experimental Models of Inherited PrP Prion Diseases. Cold Spring Harb Perspect Med 7, doi:10.1101/cshperspect.a027151 (2017).

27 Chiesa, R., Piccardo, P., Ghetti, B. \& Harris, D. A. Neurological illness in transgenic mice expressing a prion protein with an insertional mutation. Neuron 21, 1339-1351 (1998).

28 Owen, F. et al. A dementing illness associated with a novel insertion in the prion protein gene. Brain Res Mol Brain Res 13, 155-157 (1992).

29 Krasemann, S. et al. Prion disease associated with a novel nine octapeptide repeat insertion in the PRNP gene. Brain Res Mol Brain Res 34, 173-176 (1995).

30 Duchen, L. W., Poulter, M. \& Harding, A. E. Dementia associated with a 216 base pair insertion in the prion protein gene. Clinical and neuropathological features. Brain 116 ( Pt 3), 555-567 (1993).

31 Capellari, S., Zaidi, S. I., Long, A. C., Kwon, E. E. \& Petersen, R. B. The Thr183Ala Mutation, Not the Loss of the First Glycosylation Site, Alters the Physical Properties of the Prion Protein. J Alzheimers Dis 2, 27-35, doi:10.3233/jad-2000-2104 (2000).

32 Gu, Y., Verghese, S., Bose, S., Mohan, M. \& Singh, N. Mutant prion protein D202N associated with familial prion disease is retained in the endoplasmic reticulum and forms 'curly' intracellular aggregates. $J$ Mol Neurosci 32, 90-96, doi:10.1007/s12031-007-0023-6 (2007).

33 Kiachopoulos, S., Heske, J., Tatzelt, J. \& Winklhofer, K. F. Misfolding of the prion protein at the plasma membrane induces endocytosis, intracellular retention and degradation. Traffic 5, 426-436, doi:10.1111/j.1398-9219.2004.00185.x (2004). 
34 Nixon, R., Camicioli, R., Jamison, K., Cervenakova, L. \& Mastrianni, J. A. The PRNP-V180I mutation is associated with abnormally glycosylated PrPCJD and Intracellular PrP accumulations. Presented at XIVth International Congress of Neuropathology Scientific Programme. Brain Pathol. 10, 670 (2000).

35 Muramoto, T. et al. Heritable disorder resembling neuronal storage disease in mice expressing prion protein with deletion of an alpha-helix. Nat Med 3, 750755, doi:10.1038/nm0797-750 (1997).

36 Medrano, A. Z., Barmada, S. J., Biasini, E. \& Harris, D. A. GFP-tagged mutant prion protein forms intra-axonal aggregates in transgenic mice. Neurobiol Dis 31, 20-32, doi:10.1016/j.nbd.2008.03.006 (2008).

37 Ma, J. \& Lindquist, S. Wild-type PrP and a mutant associated with prion disease are subject to retrograde transport and proteasome degradation. Proc Natl Acad Sci U S A 98, 14955-14960, doi:10.1073/pnas.011578098 (2001).

38 Godsave, S. F. et al. Cryo-immunogold electron microscopy for prions: toward identification of a conversion site. J Neurosci 28, 12489-12499, doi:10.1523/JNEUROSCI.4474-08.2008 (2008).

39 Borchelt, D. R., Taraboulos, A. \& Prusiner, S. B. Evidence for synthesis of scrapie prion proteins in the endocytic pathway. J Biol Chem 267, 16188-16199 (1992).

40 Harris, D. A. Trafficking, turnover and membrane topology of PrP. Br. Med. Bull. 66, 71-85 (2003).

41 Peters, P. J. et al. Trafficking of prion proteins through a caveolae-mediated endosomal pathway. The Journal of cell biology 162, 703-717 (2003).

42 Hegde, R. S. \& Rane, N. S. Prion protein trafficking and the development of neurodegeneration. Trends Neurosci 26, 337-339, doi:10.1016/S01662236(03)00143-7 (2003).

43 Prado, M. A. et al. PrPc on the road: trafficking of the cellular prion protein. $J$ Neurochem 88, 769-781, doi:10.1046/j.1471-4159.2003.02199.x (2004).

44 Caughey, B. \& Baron, G. S. Prions and their partners in crime. Nature 443, 803810, doi:10.1038/nature05294 (2006).

45 Kang, Y. S., Zhao, X., Lovaas, J., Eisenberg, E. \& Greene, L. E. Clathrinindependent internalization of normal cellular prion protein in neuroblastoma cells is associated with the Arf6 pathway. J Cell Sci 122, 4062-4069, doi:10.1242/jcs.046292 (2009).

46 Sunyach, C. et al. The mechanism of internalization of glycosylphosphatidylinositol-anchored prion protein. EMBO J 22, 3591-3601, doi:10.1093/emboj/cdg344 (2003). 
47 Satpute-Krishnan, P. et al. ER stress-induced clearance of misfolded GPIanchored proteins via the secretory pathway. Cell 158, 522-533, doi:10.1016/j.cell.2014.06.026 (2014).

48 Zavodszky, E. \& Hegde, R. S. Misfolded GPI-anchored proteins are escorted through the secretory pathway by ER-derived factors. Elife 8, doi:ARTN e46740 10.7554/eLife.46740 (2019).

49 Shearin, H. \& Bessen, R. A. Axonal and transynaptic spread of prions. J Virol 88, 8640-8655, doi:10.1128/JVI.00378-14 (2014).

50 Encalada, S. E., Szpankowski, L., Xia, C. H. \& Goldstein, L. S. Stable kinesin and dynein assemblies drive the axonal transport of mammalian prion protein vesicles. Cell 144, 551-565, doi:10.1016/j.cell.2011.01.021 (2011).

51 Klassen, M. P. et al. An Arf-like small G protein, ARL-8, promotes the axonal transport of presynaptic cargoes by suppressing vesicle aggregation. Neuron 66, 710-723, doi:10.1016/j.neuron.2010.04.033 (2010).

52 Bagshaw, R. D., Callahan, J. W. \& Mahuran, D. J. The Arf-family protein, Arl8b, is involved in the spatial distribution of lysosomes. Biochemical and biophysical research communications 344, 1186-1191, doi:10.1016/j.bbrc.2006.03.221 (2006).

53 Hofmann, I. \& Munro, S. An N-terminally acetylated Arf-like GTPase is localised to lysosomes and affects their motility. J Cell Sci 119, 1494-1503, doi:10.1242/jcs.02958 (2006).

54 Rosa-Ferreira, C. \& Munro, S. Arl8 and SKIP act together to link lysosomes to kinesin-1. Dev Cell 21, 1171-1178, doi:10.1016/j.devcel.2011.10.007 (2011).

55 Pols, M. S. et al. hVps41 and VAMP7 function in direct TGN to late endosome transport of lysosomal membrane proteins. Nat Commun 4, 1361, doi:10.1038/ncomms2360 (2013).

56 Khatter, D., Sindhwani, A. \& Sharma, M. Arf-like GTPase Arl8: Moving from the periphery to the center of lysosomal biology. Cellular logistics 5, e1086501, doi:10.1080/21592799.2015.1086501 (2015).

57 Korolchuk, V. I. et al. Lysosomal positioning coordinates cellular nutrient responses. Nat Cell Biol 13, 453-460, doi:10.1038/ncb2204 (2011).

58 Chiesa, R. et al. Accumulation of protease-resistant prion protein $(\mathrm{PrP})$ and apoptosis of cerebellar granule cells in transgenic mice expressing a PrP insertional mutation. Proc Natl Acad Sci U S A 97, 5574-5579 (2000).

59 Drisaldi, B. et al. Mutant PrP is delayed in its exit from the endoplasmic reticulum, but neither wild-type nor mutant PrP undergoes retrotranslocation prior to proteasomal degradation. J Biol Chem 278, 21732-21743, doi:10.1074/jbc.M213247200 (2003). 
60 Barmada, S., Piccardo, P., Yamaguchi, K., Ghetti, B. \& Harris, D. A. GFP-tagged prion protein is correctly localized and functionally active in the brains of transgenic mice. Neurobiol Dis 16, 527-537, doi:10.1016/j.nbd.2004.05.005 (2004).

61 Borchelt, D. R. et al. A vector for expressing foreign genes in the brains and hearts of transgenic mice. Genetic analysis : biomolecular engineering 13, 159163 (1996).

62 Kang, S. W. et al. Substrate-specific translocational attenuation during ER stress defines a pre-emptive quality control pathway. Cell 127, 999-1013, doi:10.1016/j.cell.2006.10.032 (2006).

63 Chiesa, R. et al. Molecular distinction between pathogenic and infectious properties of the prion protein. J Virol 77, 7611-7622 (2003).

64 Plate, L. et al. Small molecule proteostasis regulators that reprogram the ER to reduce extracellular protein aggregation. Elife 5, doi:10.7554/eLife.15550 (2016).

65 Klausner, R. D., Donaldson, J. G. \& Lippincott-Schwartz, J. Brefeldin A: insights into the control of membrane traffic and organelle structure. J Cell Biol 116, 1071-1080, doi:10.1083/jcb.116.5.1071 (1992).

66 Sekine-Aizawa, Y. \& Huganir, R. L. Imaging of receptor trafficking by using alpha-bungarotoxin-binding-site-tagged receptors. Proc Natl Acad Sci U S A 101, 17114-17119, doi:10.1073/pnas.0407563101 (2004).

67 Ivanova, L., Barmada, S., Kummer, T. \& Harris, D. A. Mutant prion proteins are partially retained in the endoplasmic reticulum. J Biol Chem 276, 42409-42421, doi:10.1074/jbc.M106928200 (2001).

68 Farias, G. G., Guardia, C. M., De Pace, R., Britt, D. J. \& Bonifacino, J. S. $\mathrm{BORC/kinesin-1} \mathrm{ensemble} \mathrm{drives} \mathrm{polarized} \mathrm{transport} \mathrm{of} \mathrm{lysosomes} \mathrm{into} \mathrm{the} \mathrm{axon.}$ Proc Natl Acad Sci U S A 114, E2955-E2964, doi:10.1073/pnas.1616363114 (2017).

69 Medori, R. et al. Fatal familial insomnia, a prion disease with a mutation at codon 178 of the prion protein gene. The New England journal of medicine 326, 444449 (1992).

70 Neumann, S., Chassefeyre, R., Campbell, G. E. \& Encalada, S. E. KymoAnalyzer: a software tool for the quantitative analysis of intracellular transport in neurons. Traffic 18, 71-88, doi:10.1111/tra.12456 (2017).

71 El Meskini, R. et al. A signal sequence is sufficient for green fluorescent protein to be routed to regulated secretory granules. Endocrinology 142, 864-873 (2001).

72 Cui, J. et al. Targeted inactivation of kinesin-1 in pancreatic beta-cells in vivo leads to insulin secretory deficiency. Diabetes 60, 320-330, doi:10.2337/db091078 (2011). 
73 Macia, E. et al. Dynasore, a cell-permeable inhibitor of dynamin. Dev Cell 10, 839-850, doi:10.1016/j.devcel.2006.04.002 (2006).

74 Luzio, J. P. et al. Membrane dynamics and the biogenesis of lysosomes. Mol Membr Biol 20, 141-154, doi:10.1080/0968768031000089546 (2003).

75 Arvan, P., Zhao, X., Ramos-Castaneda, J. \& Chang, A. Secretory pathway quality control operating in Golgi, plasmalemmal, and endosomal systems. Traffic 3, 771-780 (2002).

76 Couve, A. \& Hetz, C. RESETing ER proteostasis: selective stress pathway hidden in the secretory route. EMBO J 33, 2444-2446, doi:10.15252/embj.201489845 (2014).

77 Rosa-Ferreira, C., Sweeney, S. T. \& Munro, S. The small G protein Arl8 contributes to lysosomal function and long-range axonal transport in Drosophila. Biol Open 7, doi:10.1242/bio.035964 (2018).

78 Sanger, A. et al. SKIP controls lysosome positioning using a composite kinesin-1 heavy and light chain-binding domain. J Cell Sci 130, 1637-1651, doi:10.1242/jcs.198267 (2017).

79 Ishida, M., Ohbayashi, N. \& Fukuda, M. Rab1A regulates anterograde melanosome transport by recruiting kinesin-1 to melanosomes through interaction with SKIP. Sci Rep 5, 8238, doi:10.1038/srep08238 (2015).

80 Khatter, D. et al. The small GTPase Arl8b regulates assembly of the mammalian HOPS complex on lysosomes. J Cell Sci 128, 1746-1761, doi:10.1242/jcs.162651 (2015).

81 Pamarthy, S., Kulshrestha, A., Katara, G. K. \& Beaman, K. D. The curious case of vacuolar ATPase: regulation of signaling pathways. Mol Cancer 17, 41, doi:10.1186/s12943-018-0811-3 (2018).

82 Senatore, A. et al. Mutant PrP suppresses glutamatergic neurotransmission in cerebellar granule neurons by impairing membrane delivery of VGCC alpha(2)delta-1 Subunit. Neuron 74, 300-313, doi:10.1016/j.neuron.2012.02.027 (2012).

$83 \mathrm{Pu}, \mathrm{J}$. et al. BORC, a multisubunit complex that regulates lysosome positioning. Dev Cell 33, 176-188, doi:10.1016/j.devcel.2015.02.011 (2015).

84 Filipek, P. A. et al. LAMTOR/Ragulator is a negative regulator of Arl8b- and BORC-dependent late endosomal positioning. J Cell Biol 216, 4199-4215, doi:10.1083/jcb.201703061 (2017).

85 Takalo, M., Salminen, A., Soininen, H., Hiltunen, M. \& Haapasalo, A. Protein aggregation and degradation mechanisms in neurodegenerative diseases. Am J Neurodegener Dis 2, 1-14 (2013). 
86 Kristiansen, M. et al. Disease-related prion protein forms aggresomes in neuronal cells leading to caspase activation and apoptosis. J Biol Chem 280, 3885138861, doi:10.1074/jbc.M506600200 (2005).

87 Ashok, A. \& Hegde, R. S. Selective processing and metabolism of diseasecausing mutant prion proteins. PLoS Pathog 5, e1000479, doi:10.1371/journal.ppat.1000479 (2009).

88 Velier, J. et al. Wild-type and mutant huntingtins function in vesicle trafficking in the secretory and endocytic pathways. Exp Neurol 152, 34-40, doi:10.1006/exnr.1998.6832 (1998).

89 van Weering, J. R. T. \& Scheper, W. Endolysosome and Autolysosome Dysfunction in Alzheimer's Disease: Where Intracellular and Extracellular Meet. CNS Drugs 33, 639-648, doi:10.1007/s40263-019-00643-1 (2019).

90 Vidyadhara, D. J., Lee, J. E. \& Chandra, S. S. Role of the endolysosomal system in Parkinson's disease. J Neurochem 150, 487-506, doi:10.1111/jnc.14820 (2019).

91 Subach, F. V. et al. Photoactivatable mCherry for high-resolution two-color fluorescence microscopy. Nat Methods 6, 153-159, doi:10.1038/nmeth.1298 (2009).

92 Maday, S. \& Holzbaur, E. L. Compartment-Specific Regulation of Autophagy in Primary Neurons. J Neurosci 36, 5933-5945, doi:10.1523/JNEUROSCI.440115.2016 (2016).

93 Nakagawa, T. et al. Quaternary structure, protein dynamics, and synaptic function of SAP97 controlled by L27 domain interactions. Neuron 44, 453-467, doi:10.1016/j.neuron.2004.10.012 (2004).

94 Dunn, S. et al. Differential trafficking of Kif5c on tyrosinated and detyrosinated microtubules in live cells. J Cell Sci 121, 1085-1095, doi:10.1242/jcs.026492 (2008).

95 McClure, C., Cole, K. L., Wulff, P., Klugmann, M. \& Murray, A. J. Production and titering of recombinant adeno-associated viral vectors. Journal of visualized experiments : JoVE, e3348, doi:10.3791/3348 (2011).

96 Williamson, R. A. et al. Mapping the prion protein using recombinant antibodies. J Virol 72, 9413-9418 (1998).

97 Gustafsson, N. et al. Fast live-cell conventional fluorophore nanoscopy with ImageJ through super-resolution radial fluctuations. Nat Commun 7, 12471, doi:10.1038/ncomms12471 (2016).

98 Burel, A. et al. A targeted 3D EM and correlative microscopy method using SEM array tomography. Development 145, doi:10.1242/dev.160879 (2018). 
99 Saalfeld, S., Fetter, R., Cardona, A. \& Tomancak, P. Elastic volume reconstruction from series of ultra-thin microscopy sections. Nat Methods 9, 717720, doi:10.1038/nmeth.2072 (2012).

100 Schneider, C. A., Rasband, W. S. \& Eliceiri, K. W. NIH Image to ImageJ: 25 years of image analysis. Nat Methods 9, 671-675, doi:10.1038/nmeth.2089 (2012).

101 Sage, D. et al. DeconvolutionLab2: An open-source software for deconvolution microscopy. Methods (San Diego, Calif.) 115, 28-41, doi:10.1016/j.ymeth.2016.12.015 (2017).

\section{ACKNOWLEDGEMENTS}

We thank Malcom Wood at the Scripps Research EM core for performing the SEM experiments; David Harris (Boston U.), Lars Plate (Vanderbilt U.), Larry S. B. Goldstein (U. California San Diego), and Vladislav Verkhusha (Albert Einstein College of Medicine) for sharing reagents; Susan Ackerman, Jeff Kelly, Justine Lebeau, Malene Hansen, Michael Petrascheck, Luke Wiseman, Federico Zampa, Giordano Lippi, George Campbell, Kayal Madhivanan, and all members of the Encalada Lab for advice and valuable discussions and comments. This work was supported, in part, by a NIH/NIA R01AG049483 grant; by the Glenn Foundation for Medical Research Award for Research in Biological Mechanisms of Aging; by a New Scholar in Aging Award from the Lawrence Ellison Foundation; and by a Baxter Family Foundation award to S.E.E. R.C. was supported by a fellowship from the George E. Hewitt Foundation for Medical Research. T.C. was supported by a Royal Thai Government Scholarship from the Development and Promotion of Science and Technology Talents Project (DPST). U.M., S.W.N., and L.R.A. are supported by the Waitt Foundation, Core Grant application NCl CCSG (CA014195), NSF NeuroNex Award No. 2014862, and NIH/NIDCD R21 award DC018237.

\section{AUTHOR CONTRIBUTIONS}


S.E.E., and R.C. conceptualized the research. R.C., A.V., T.C, S.W.N, L.R.A. performed the research. A.V., T.C., S.W.N, L.R.A. validated the research. R.C., A.V., T.C., S.W.N, L.R.A., U.M., S.E.E., analyzed the data. R.C., S.E.E wrote the original draft. S.E.E., T.C., A.V., U.M. reviewed, edited manuscript.

\section{COMPETING INTERESTS}

The authors declare no competing interests.

\section{MATERIALS AND CORRESPONDANCE}

Should be addressed to S.E.E. 


\section{FIGURES}

a

PrP $^{\mathrm{WT}}$-mCherry

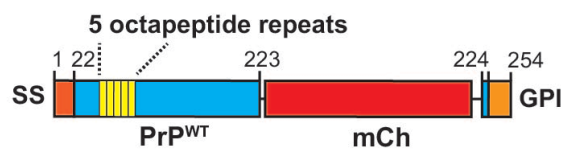

b
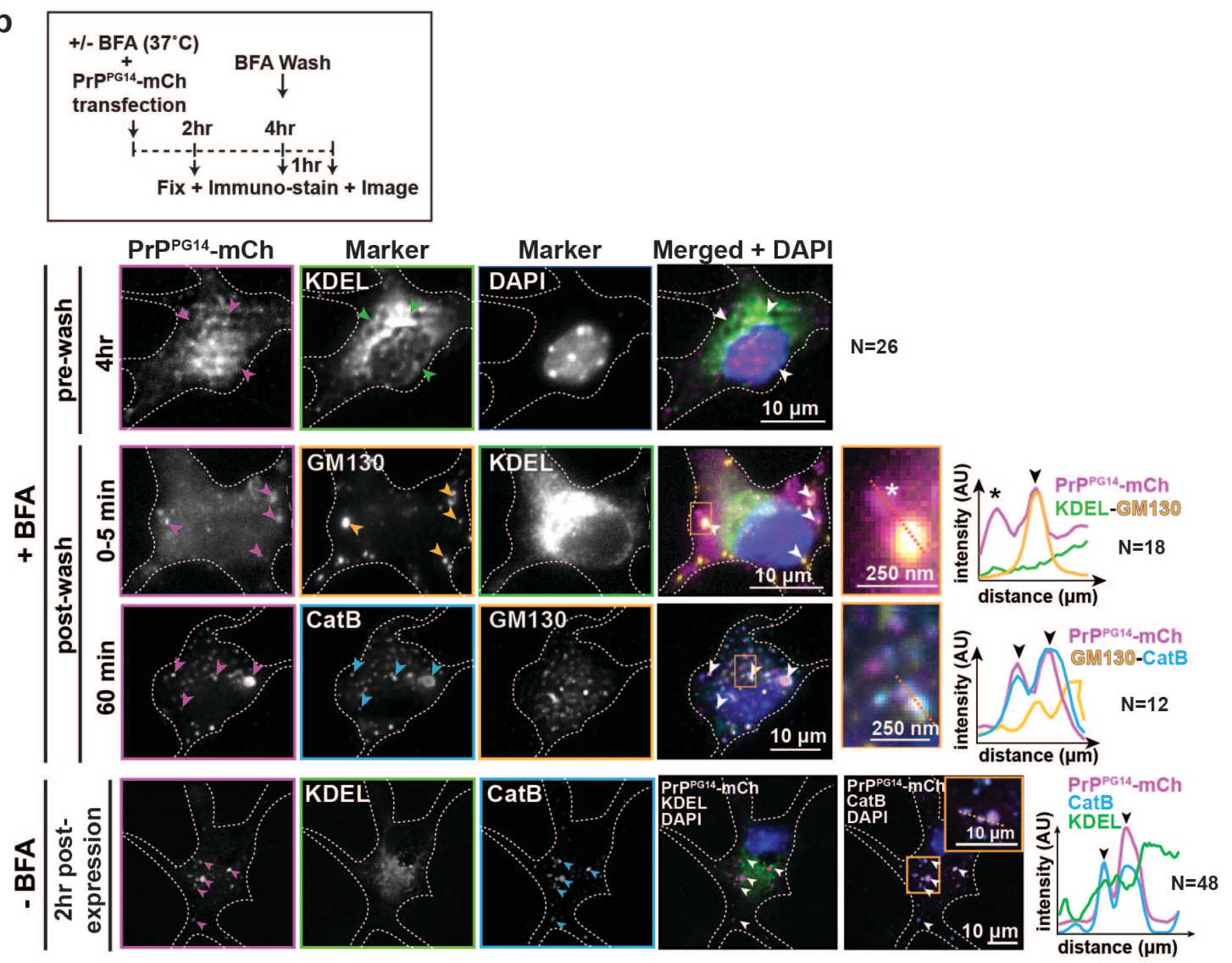

C

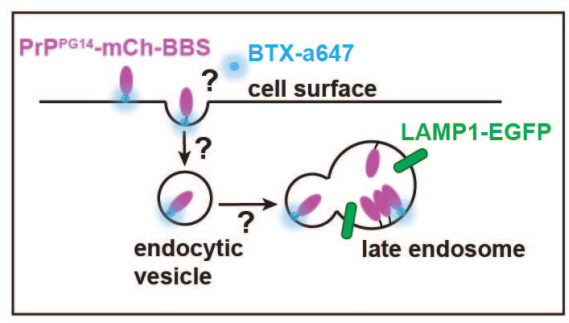

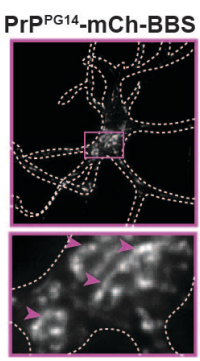
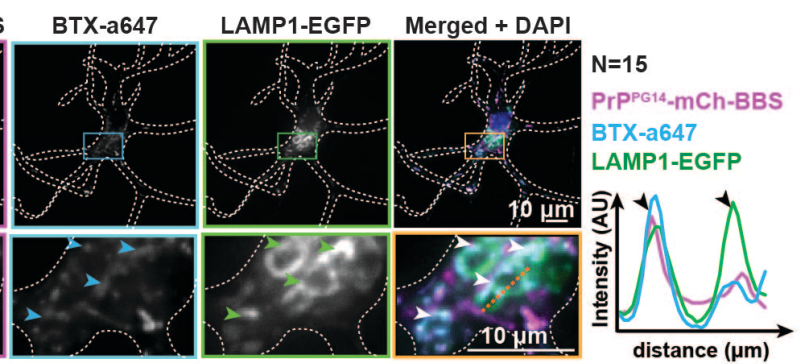

d
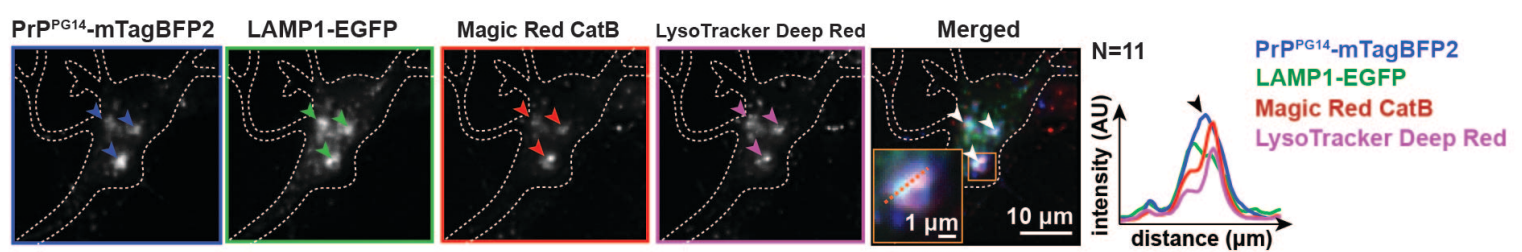
Figure 1. Neuronal PrP ${ }^{\mathrm{PG} 14}$ undergoes lysosomal clearance. a, Schematic of PrP ${ }^{W T}$ $\mathrm{mCh}$ and $\mathrm{PrP}^{\mathrm{PG} 14}$-mCh constructs. SS: signal sequence, GPI: GPI anchor. b, Experimental outline of BFA assay (top). Representative images of soma of different neurons expressing $\mathrm{PrP}^{\mathrm{PG} 14}$-mCh and stained with antibodies against ER (KDEL), Golgi (GM130), lysosomes (CatB) or with DAPI nuclear marker at indicated time points and conditions (bottom). c, Outline of $\mathrm{PrP}^{\mathrm{PG} 14}-\mathrm{mCh}-\mathrm{BBS}$ internalization assay (left). Representative images of the soma of a neuron cotransfected with $\operatorname{PrP}^{\mathrm{PG} 14}-\mathrm{mCh}-\mathrm{BBS}$ and LAMP1-EGFP, and labeled with BTX-a647 (right). d, Representative images of the soma of a neuron cotransfected with PrP ${ }^{\mathrm{PG} 14}$-mTagBFP2 and LAMP1-EGFP, and treated with Magic Red and LysoTracker Deep Red). Arrowheads and asterisk point to colocalization or non-colocalization events, respectively, also shown in enlarged insets and line scan intensity profiles. See also Supplementary Figure 1. 
a

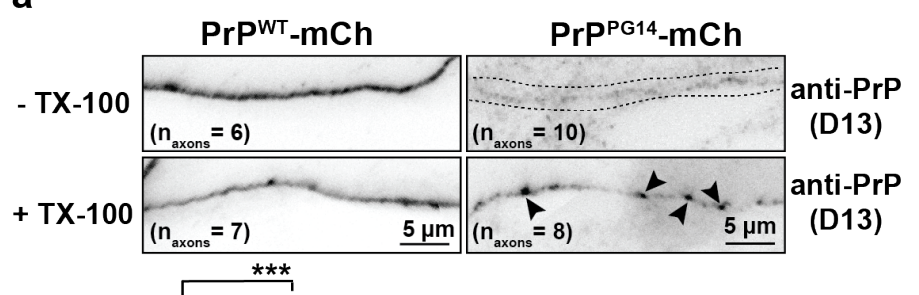

b

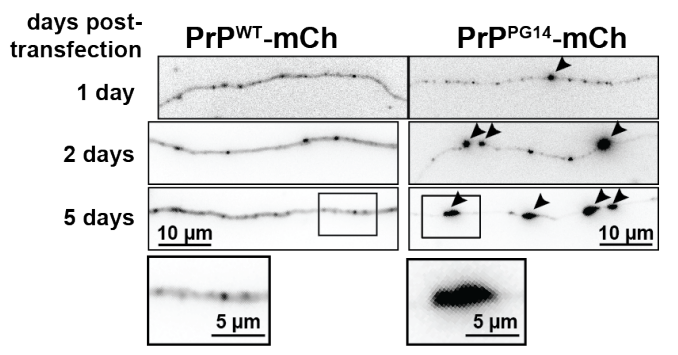

d

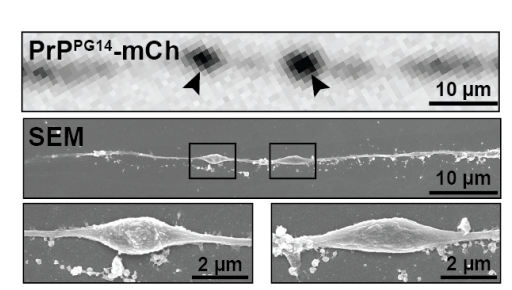

PrPWT-mCh:
1 day $\left(n_{\text {axons }}=21\right)$
2 days $\left(n_{\text {axons }}=34\right)$
5 days $\left(n_{\text {axons }}=30\right)$
PrPPG14-mCh:
1 day $\left(n_{\text {axons }}=51\right)$
2 days $\left(n_{\text {axons }}=35\right)$
5 days $\left(n_{\text {axons }}=40\right)$

.

e

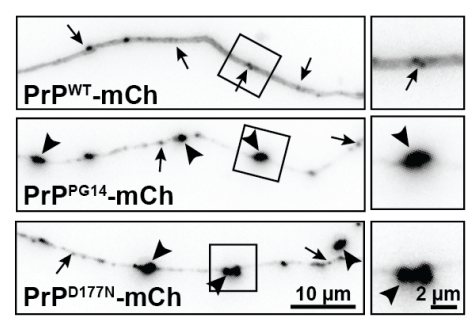

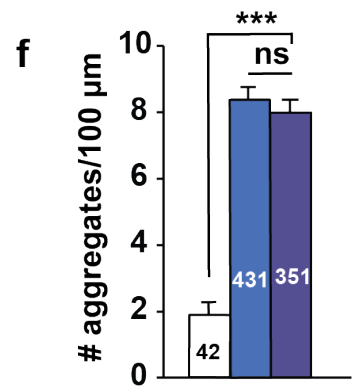

2 days post-transfection:

$\square$ PrPWT-mCh $\quad\left(\mathrm{n}_{\mathrm{axons}}=\mathbf{2 1}\right)$

$\square$ PrPPG14 $^{-m C h}\left(\mathrm{n}_{\text {axons }}=35\right)$

$\operatorname{PrPD177N}-m C h\left(n_{\text {axons }}=27\right)$

Figure 2. $\mathrm{PrP}^{\mathrm{PG} 14}$ forms intra-axonal aggregates and swellings. a,

Immunofluorescence images of axons expressing $\mathrm{PrP}^{\mathrm{WT}}-\mathrm{mCh}$ and $\mathrm{PrP}^{\mathrm{PG} 14}-\mathrm{mCh}$ in the presence or absence of detergent (TX-100). Arrowheads point to aggregates. b,

Representative images of axons expressing $\mathrm{PrP}^{\mathrm{WT}}-\mathrm{mCh}$ and $\mathrm{PrP}^{\mathrm{PG} 14}-\mathrm{mCh}$. Arrowheads

point to aggregates. Insets show enlargements. c, Quantitation of aggregate densities

from (b). $\mathrm{N}_{\text {aggregates }}$ shown inside bars. d, Correlative fluorescence (top) and SEM (middlebottom) images of $\mathrm{PrP}^{\mathrm{PG} 14}$-mCh aggregate swellings. $\mathrm{N}_{\text {swellings }}=20$. Arrowheads point to aggregates. Insets show enlargements. e, Representative images of axons expressing $\mathrm{PrP}^{\mathrm{WT}}-\mathrm{mCh}, \mathrm{PrP}^{\mathrm{PG} 14}-\mathrm{mCh}$, or $\mathrm{PrP}^{\mathrm{D} 177 \mathrm{~N}}-\mathrm{mCh} 2$ days post-transfection. Arrows point to vesicles. Arrowheads point to aggregates. f, Quantitation of aggregate densities from (e). $N_{\text {aggregates }}$ shown inside bars. All values are shown as mean \pm SEM. ${ }^{* *} p<0.01,{ }^{* * *} p<0.001$, 
bioRxiv preprint doi: https://doi.org/10.1101/2020.09.27.315846; this version posted September 28, 2020. The copyright holder for this preprint (which was not certified by peer review) is the author/funder. All rights reserved. No reuse allowed without permission.

\section{ns = not significant, Student's t-test, Šidák correction $(\mathbf{c}, \mathbf{f})$. See also Supplementary}

\section{Figure 2.}


a

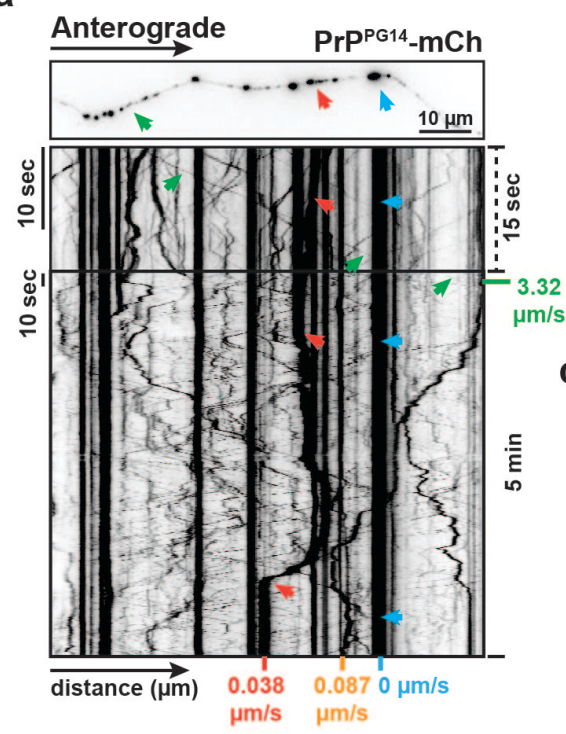

e

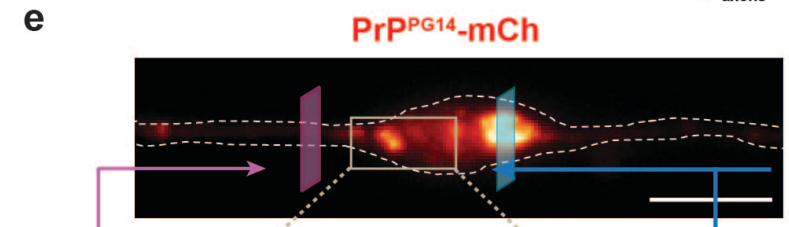

non-aggregate site volumetric reconstruction

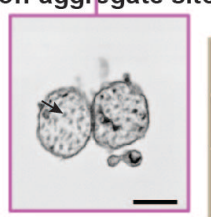
aggregate site

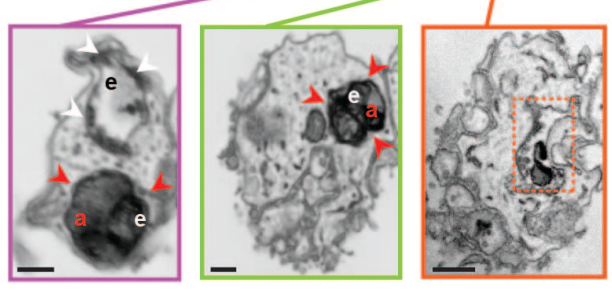

aggregate site

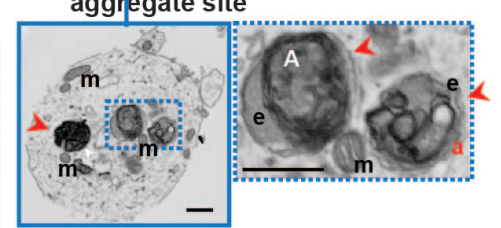

Figure 3. $\mathrm{PrP}^{\mathrm{PG} 14}$ is transported and accumulates within endo-lysosomal

compartments in axons. a, First frame of movie of an axon expressing $\mathrm{PrP}^{\mathrm{PG} 14}-\mathrm{mCh}$ (top), and corresponding kymographs (middle-bottom). Arrows point to vesicles or aggregates moving in the anterograde (green) or retrograde (red) directions, or that are stationary (blue). b, Average net velocities of $\mathrm{PrP}^{\mathrm{PG} 14}-\mathrm{mCh}$ vesicles and aggregates. $\mathbf{c}$, Representative images of axons co-expressing $\mathrm{PrP}^{\mathrm{PG} 14}-\mathrm{mCh}$ and markers for early 
endosomes (EGFP-Rab5), recycling endosomes (EGFP-Rab11), LEs (EGFP-Rab7), and endo-lysosomes (LAMP1-EGFP). Arrowheads point to colocalizing aggregates.

Scale bar $=10 \mu \mathrm{m} . \mathbf{d}$, Quantitation of aggregate densities from (c). Naggregates shown inside bars. All values are shown as mean \pm SEM. e, Correlative fluorescence (top) and Serial Sectioning Scanning Electron Microscopy (S3EM) representative cross sections (bottom). Volumetric 3D reconstruction through axonal $\mathrm{PrP}^{\mathrm{PG} 14}-\mathrm{mCh}$ aggregate site is shown with super-resolution radial fluctuation (SRRF) fluorescence overlay. Red arrowheads point to $\mathrm{PrP}^{\mathrm{PG} 14}$-mCh-positive aggregates within endosomal membranes. White arrowheads point to intraluminal vesicles. $\mathrm{m}=$ mitochondria; $\mathrm{e}=$ endosome; $\mathrm{a}=$ aggregate. Scale bar of top panel of correlative image $=10 \mu \mathrm{m}$. All other scale bars $=$ 500 nm. See also Supplementary Figure 3. 
a

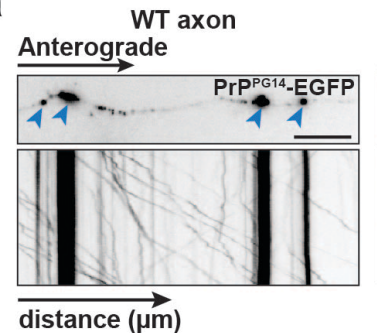

d

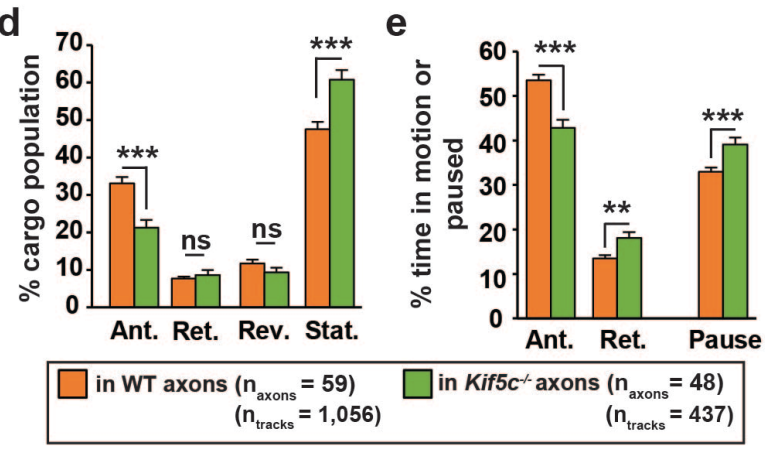

b
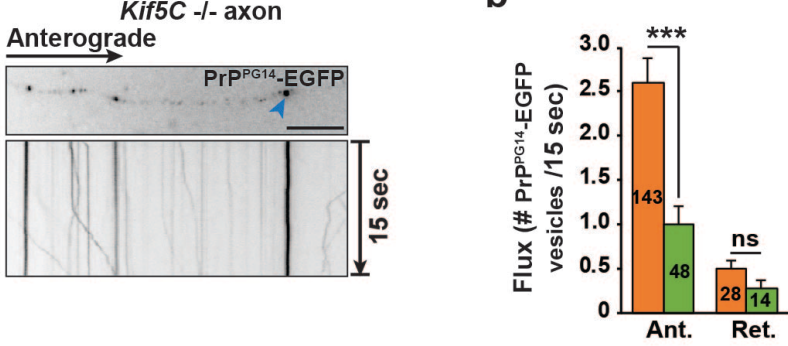

f

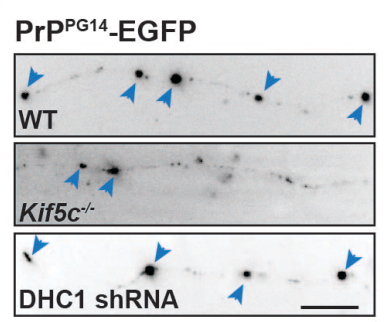

C

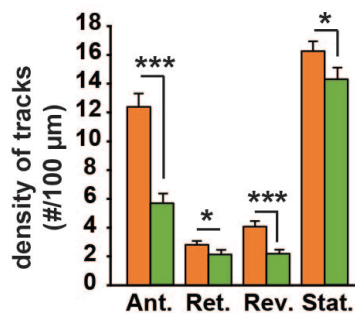

g

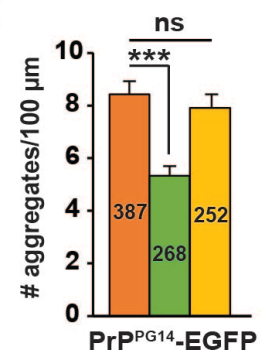

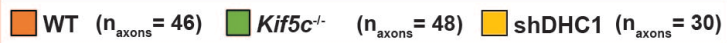

Figure 4. Kinesin-1 mediates the transport and aggregation of endoggresomes. a, Representative first frames and kymographs of PrP ${ }^{P G 14}$-EGFP vesicle transport or aggregates (arrowheads) in wild-type (WT) (left) and Kif5c ${ }^{-/-}$(right) hippocampal axons. Scale bars $=10 \mu \mathrm{m}$. b. PrP ${ }^{\mathrm{PG} 14}$-EGFP vesicle flux quantitation. $\mathrm{N}_{\text {vesicles/aggregates }}$ are inside bars. c, Densities of PrP ${ }^{P G 14}$-EGFP vesicles in WT or Kif5c $c^{-/}$axons. d, Population breakdown of PrP ${ }^{P G 14}$-EGFP vesicles in WT and Kif5c $c^{-/}$axons. e, Percent time PrP ${ }^{P G 14}$ EGFP vesicles spent in motion or paused in WT and Kif5 $c^{-/-}$axons. f, Representative images of PrP ${ }^{\mathrm{PG} 14}$-EGFP aggregates (arrowheads) in WT, Kif5 $\mathrm{c}^{-/}$, and DHC1 shRNA axons. Scale bar $=10 \mu \mathrm{m} . \mathbf{g}$, Quantitation of PrP ${ }^{\mathrm{PG} 14}$-EGFP aggregate densities from (f). $\mathrm{N}_{\text {vesicles or aggregates }}$ are shown inside bars. Ant. = anterograde, Ret. = retrograde, Rev. $=$ reversal, Stat. $=$ stationary. All values are shown as mean \pm SEM. ${ }^{* * *} p<0.001,{ }^{* *} p<$ $0.01,{ }^{*} p<0.05, n s=$ not significant, Student's t-test. See also Supplementary Figure 4. 
a

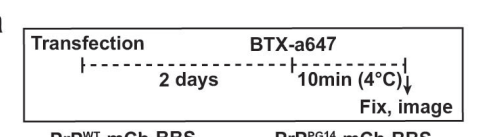

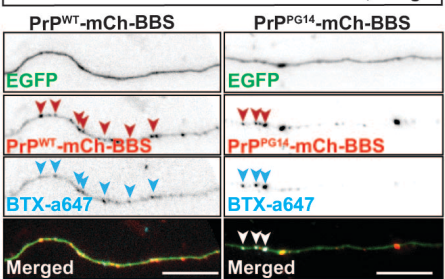

$\left(n_{\text {axons }}=7\right)$

C
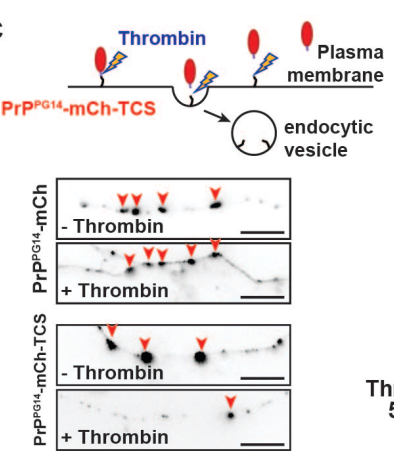

b
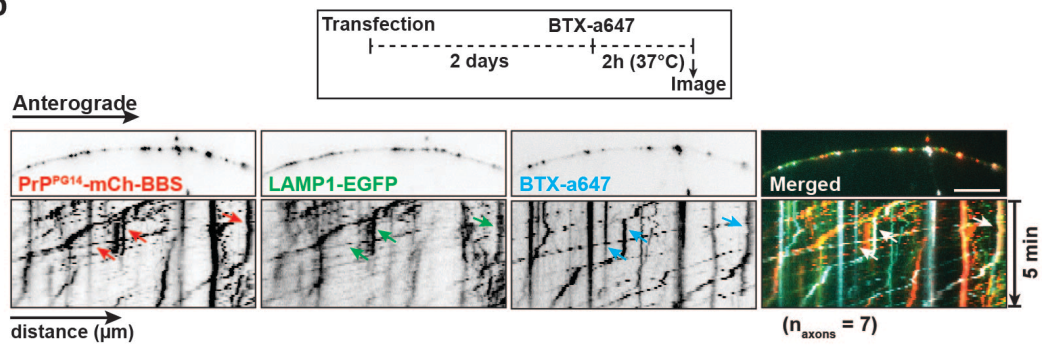

e

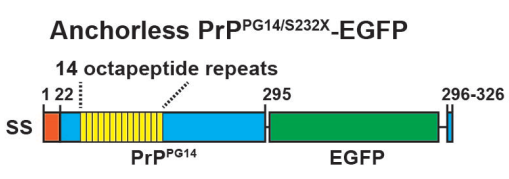

Anterograde

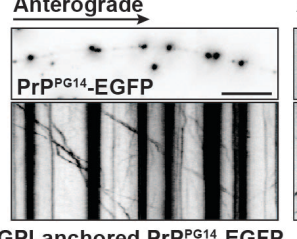
$\stackrel{\text { Anterograde }}{\longrightarrow}$

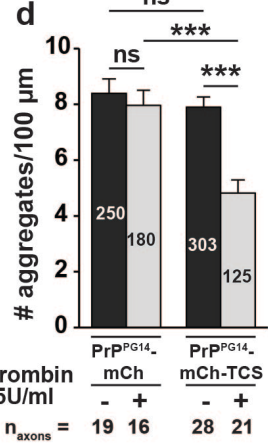

$\underset{\mathrm{GPI} \text {-anchored }}{\longrightarrow}$

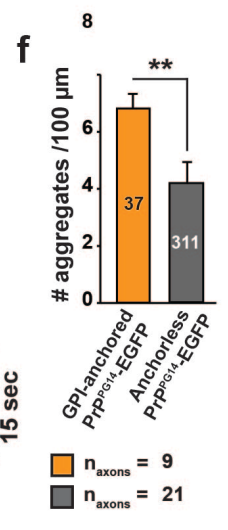

Figure 5. PrP ${ }^{\mathrm{PG} 14}$ traffics to the axonal cell surface prior to aggregation. a,

Experimental outline of 10 min BBS internalization assay (top). Representative images of axons expressing EGFP and $\mathrm{PrP}^{\mathrm{WT}}$-mCh-BBS or PrP ${ }^{\mathrm{PG} 14}$-mCh-BBS and labeled with BTX-a647 (bottom). Arrowheads point to colocalization. Scale bars $=10 \mu \mathrm{m} . \mathbf{b}$, Experimental outline of $2 \mathrm{hr}$ BBS internalization assay (top). Representative first frames of time-lapse movie, and kymographs of axons expressing PrP ${ }^{P G 14}-\mathrm{mCh}-\mathrm{BBS}$ and LAMP1-EGFP, and labeled with BTX-a647. Arrows point to cotransport. Scale bar $=10$ $\mu \mathrm{m} . \mathbf{c}$, Diagram of thrombin assay (top). Representative images of axons expressing PrP ${ }^{\mathrm{PG} 14}$-mCh or PrP ${ }^{\mathrm{PG} 14}$-mCh-TCS, with or without thrombin treatment (bottom). Arrowheads point to aggregates. Scale bars $=10 \mu \mathrm{m}$. d, Quantitation of aggregate densities from (c). $\mathrm{N}_{\text {aggregates }}$ shown inside bars. e, Schematic of anchorless PrP PG14/S232X EGFP construct (top). SS: signal sequence. Representative images and kymographs of hippocampal axons expressing PrP ${ }^{P G 14}$-EGFP or PrP ${ }^{P G 14 / S 232 X}$-EGFP (bottom). Scale 
bars $=10 \mu \mathrm{m}$. f, Quantitation of aggregate densities from (e). $N_{\text {aggregates }}$ shown inside bars. All values are shown as mean \pm SEM. ${ }^{* *} \mathrm{p}<0.001,{ }^{* *} \mathrm{p}<0.01$, Student's t-test, Tukey's correction (d). See also Supplementary Figure 5. 
a

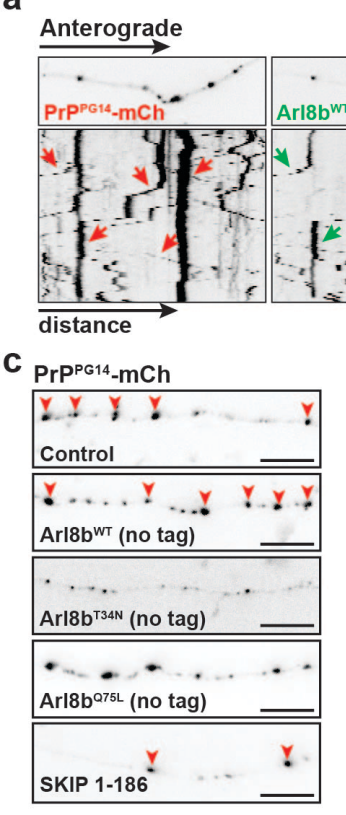

b PrPPG14-mCh Vesicles

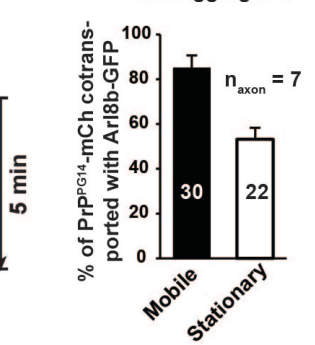

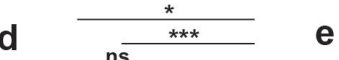

e

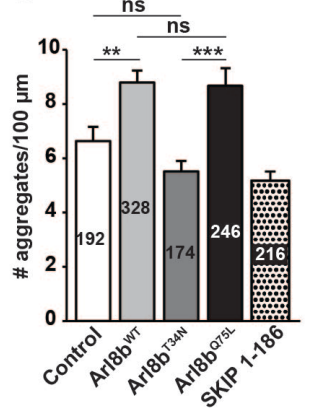

Anterograde
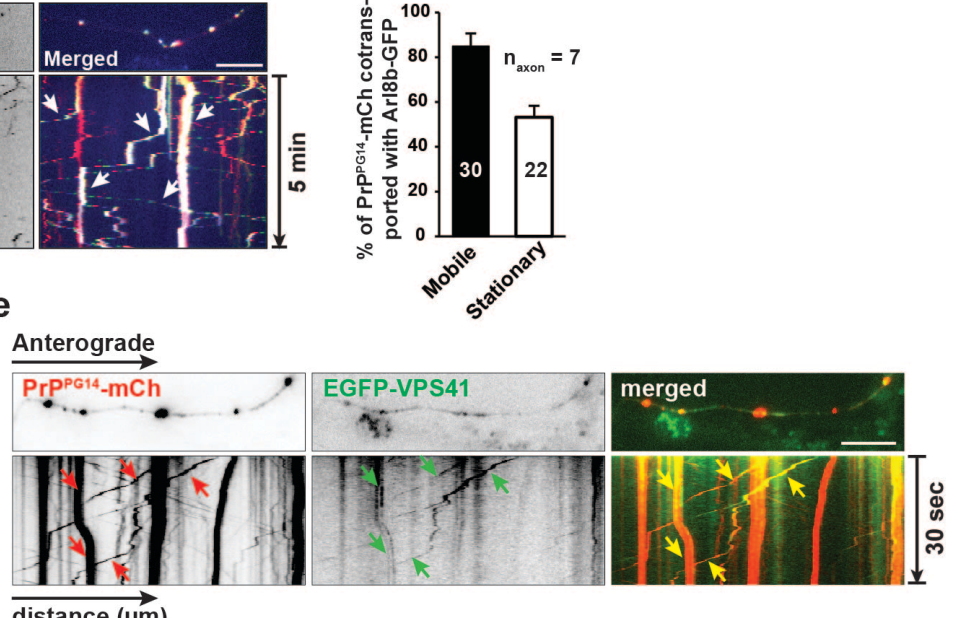

$\underset{\text { distance }(\mu \mathrm{m})}{\overrightarrow{1}}$

f

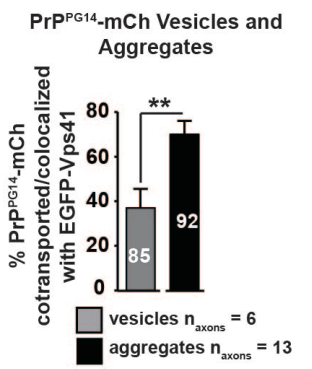

g

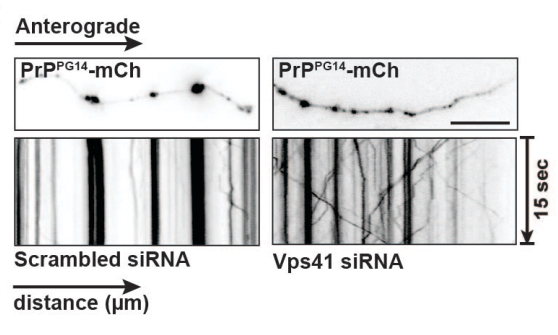

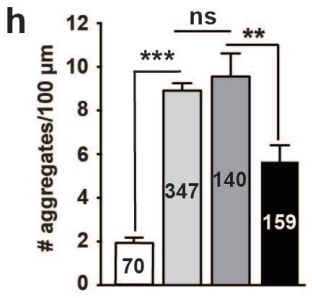

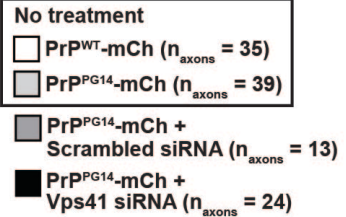

i - Arl8b ${ }^{W T}$-GFP

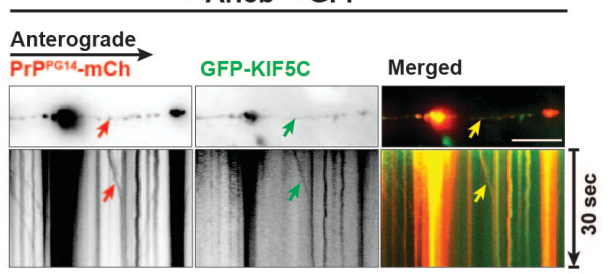

$\underset{\text { distance }}{\longrightarrow}(\mu \mathrm{m})$
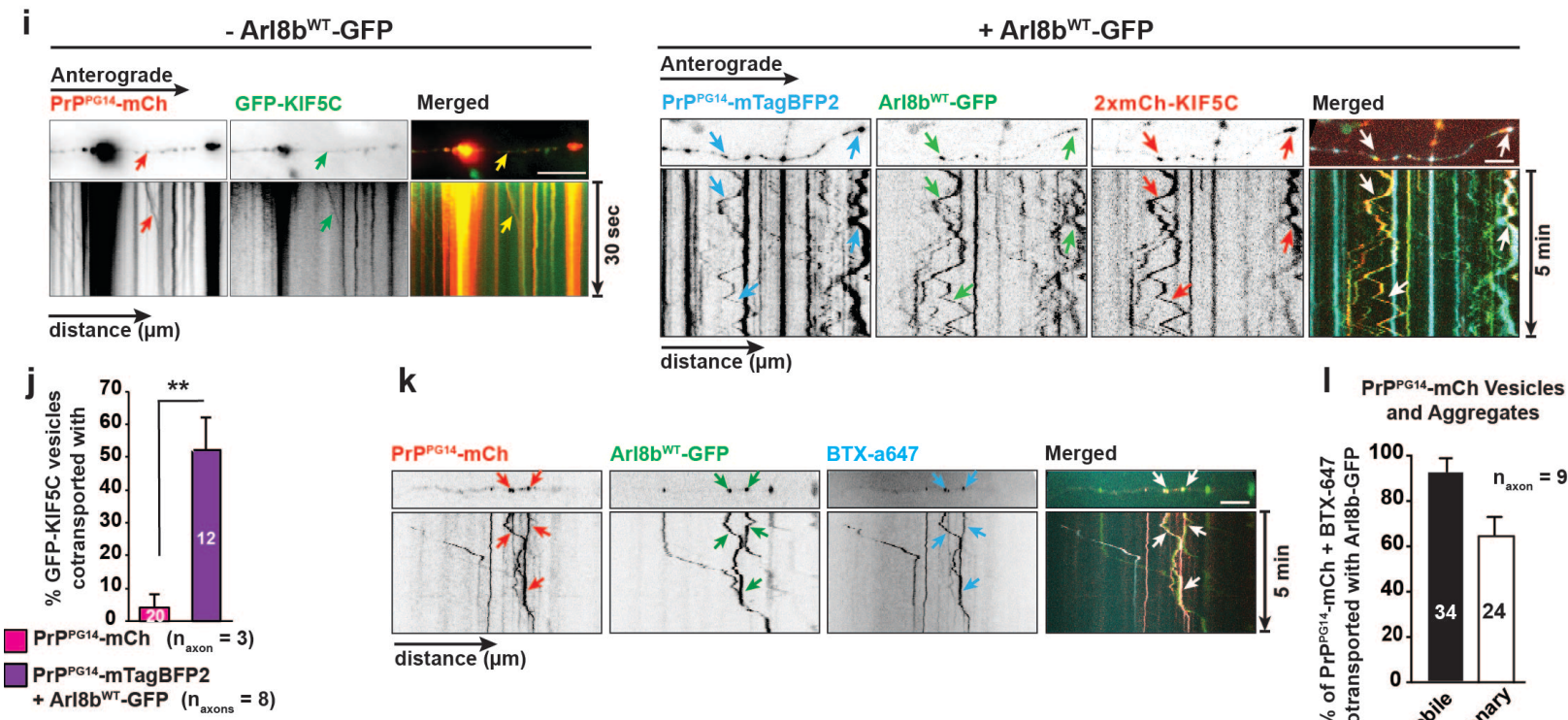

$\mathbf{k}$

$\underset{\text { distance }}{\longrightarrow}(\mu \mathrm{m})$

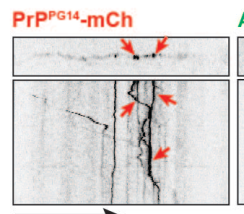

$\underset{\operatorname{distance}(\mu \mathrm{m})}{\longrightarrow}$

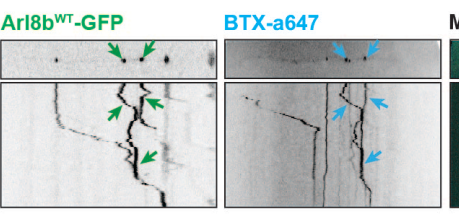

BTX-a647

Merged

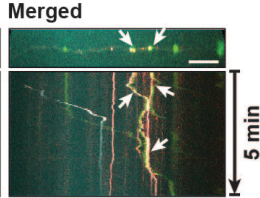

I PrPPG14-mCh Vesicles and Aggregates

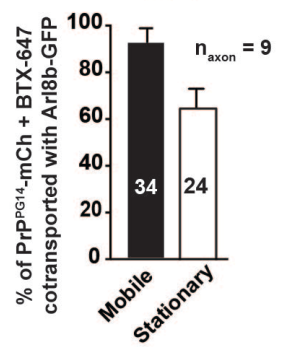


Figure 6. Arl8b drives PrP ${ }^{P G 14}$ aggregation via transport, sorting, and fusion. a, Representative first frames of time-lapse movie, and kymographs of axons expressing PrP ${ }^{P G 14}-m C h, A r l 8 b^{W T}-E G F P$, and LAMP1-mTagBFP2. Arrows point to cotransporting tracks. Scale bar $=10 \mu \mathrm{m} . \mathbf{b}$, Quantitation of cotransport between mobile or stationary

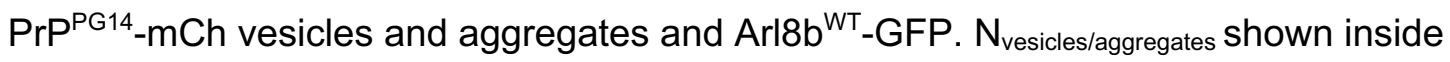
bars. c, Representative images of $\mathrm{PrP}^{\mathrm{PG} 14}$-mCh axons overexpressing empty vector (Control), Arl8b ${ }^{W T}$, dominant-negative (DN) GDP-found Arl8b ${ }^{T 34 N}$, or GTP-bound Arl8b $b^{Q 75 L}$. Scale bars $=10 \mu \mathrm{m} . \mathbf{d}$, Quantitation of aggregate densities from $(\mathbf{c})$ plus aggregate density of axons treated with SKIP 1-186. $\mathrm{N}_{\text {aggregates }}$ shown inside bars. e, Representative first frames of time-lapse movie, and kymographs of axons expressing PrP ${ }^{P G 14}-m C h$ and EGFP-VPS41. Arrows point to cotransporting tracks. Scale bar $=10$ $\mu \mathrm{m}$. f, Quantitation of $\mathrm{PrP}^{\mathrm{PG} 14}$-mCh vesicles and aggregates that cotransport/colocalize with EGFP-VPS41. N Nesicles/aggregates are shown inside bars. g, Representative first frames of time-lapse movies, and kymographs of axons co-expressing PrP ${ }^{\mathrm{PG} 14}$-mCh and scrambled or Vps41 siRNAs. Scale bar $=10 \mu \mathrm{m} . \mathbf{h}$, Quantitation of aggregate densities from (g). Naggregates are shown inside bars. i, Representative first frames of time-lapse movie, and kymographs of axons expressing PrP ${ }^{\mathrm{PG} 14}$-mCh and GFP-KIF5C (left), and of those expressing PrP ${ }^{\mathrm{PG} 14}$-mTagBFP2, Arl8b ${ }^{\mathrm{WT}}$-GFP, and 2xmCh-KIF5C (right). Arrows point to cotransporting vesicles/aggregates. Scale bars $=10 \mu \mathrm{m}$. j, Quantitation of cotransport of (i). N $N_{\text {vesicles }}$ are shown inside bars. $\mathbf{k}$, Representative first frames of timelapse movie, and kymographs of axons expressing PrP ${ }^{\mathrm{PG} 14}-\mathrm{mCh}-\mathrm{BBS}, \mathrm{Arl8b} \mathrm{b}^{\mathrm{WT}}$-GFP, and labeled with BTX-a647. Arrows point to col-transporting vesicles. Scale bar $=10 \mu \mathrm{m}$. I, Quantitation of cotransport of BTX-a647-labeled PrP ${ }^{\mathrm{PG} 14}-\mathrm{mCh}-\mathrm{BBS}$ vesicles and aggregates with Arl8b $\mathrm{b}^{\mathrm{WT}}$-GFP from (k). $\mathrm{N}_{\text {vesicles }}$ are shown inside bars. All values are shown as mean \pm SEM. ${ }^{* * *} p<0.001,{ }^{* *} p<0.01,{ }^{*} p<0.05$, ns $=$ not significant, Student's $t-$ 
bioRxiv preprint doi: https://doi.org/10.1101/2020.09.27.315846; this version posted September 28, 2020. The copyright holder for this preprint (which was not certified by peer review) is the author/funder. All rights reserved. No reuse allowed without permission.

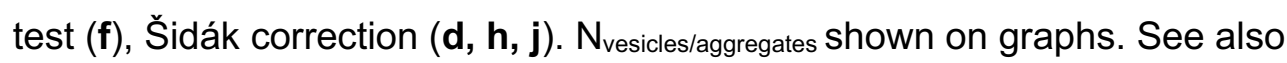

\section{Supplementary Figures 6 and 7.}


a

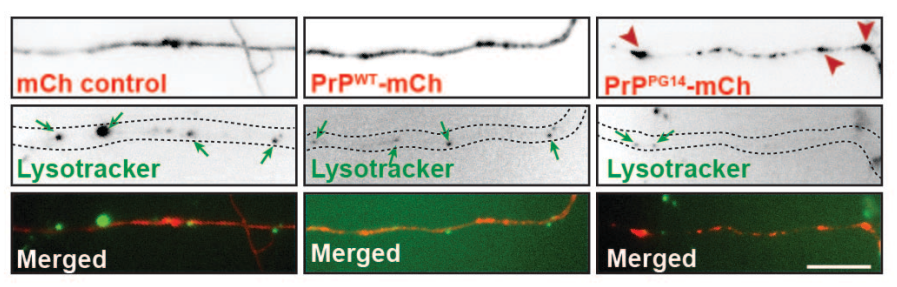

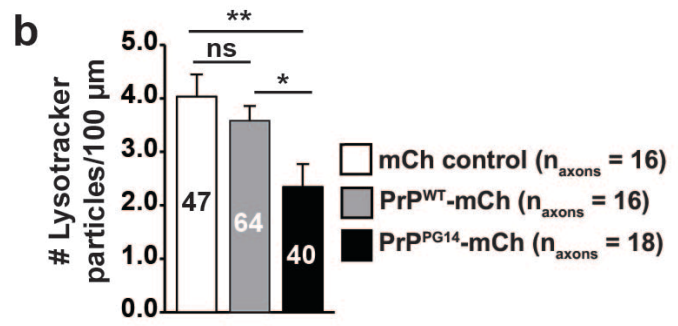
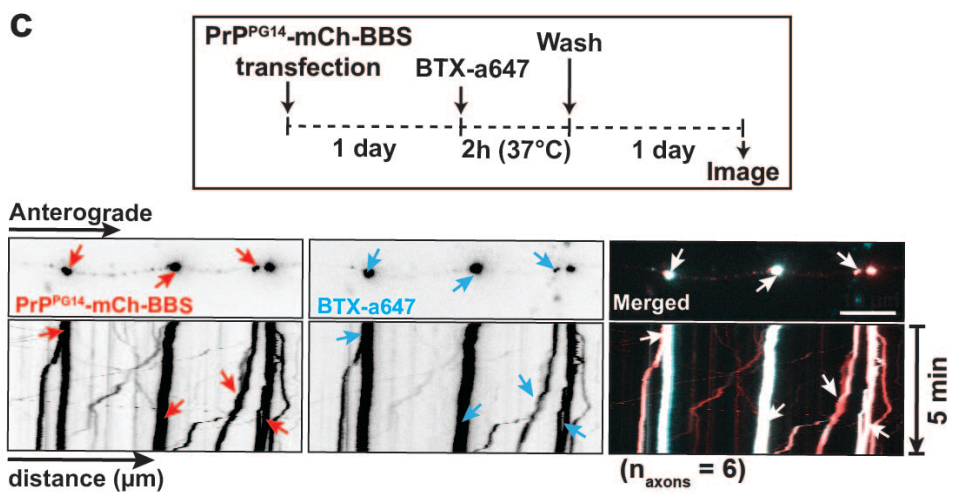

$\overrightarrow{\text { distance }(\mu \mathrm{m})}$

$\left(n_{\text {axons }}=6\right)$

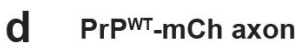
Anterograde

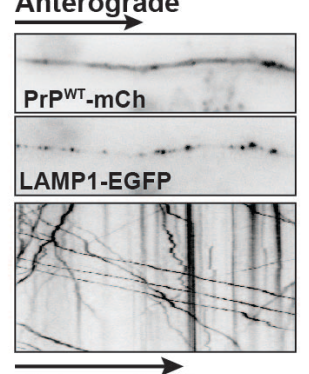

$\overrightarrow{\text { distance }(\mu \mathrm{m})}$
PrPPG14-mCh axon Anterograde

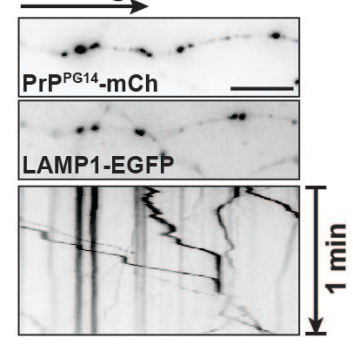

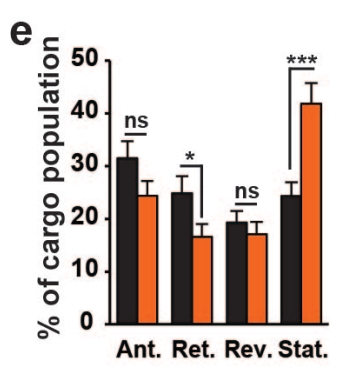

Ant. Ret. Rev. Stat.
LAMP1-EGFP

f
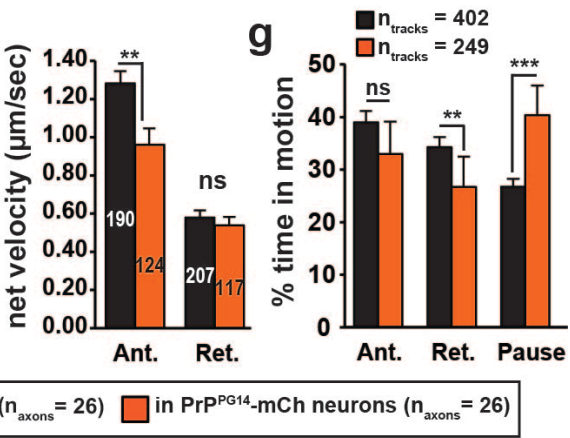

Figure 7. Impaired lysosomal maturation and retrograde transport of PrP ${ }^{P G 14}$

endoggresomes. a, Representative images of Lysotracker-positive vesicles (arrows) in mCh control, $\mathrm{PrP}^{\mathrm{WT}}-\mathrm{mCh}$, or $\mathrm{PrP}^{\mathrm{PG} 14}$-mCh-expressing axons. Arrowheads point to aggregates. Scale bar $=10 \mu \mathrm{m} . \mathbf{b}$, Quantitation of Lysotracker vesicle densities from (a). $\mathrm{N}_{\text {vesicles }}$ are shown inside bars. c, Experimental outline of BTX assay (top).

Representative first frames of time-lapse movie, and kymographs of BTX-a647-labeled $\mathrm{PrP}^{\mathrm{PG} 14}-\mathrm{mCh}-\mathrm{BBS}$ axons. Arrows point to cotransporting tracks. Scale bar $=10 \mu \mathrm{m}$. d, Representative images of $\mathrm{PrP}^{\mathrm{WT}}$-mCh or PrP ${ }^{\mathrm{PG} 14}$-mCh axons (top), and LAMP1-EGFP 
first frames of time-lapse movie (middle), and kymographs (bottom). Scale bar $=10 \mu \mathrm{m}$.

e, Population breakdown of LAMP1-EGFP vesicles in $\mathrm{PrP}^{\mathrm{WT}}-\mathrm{mCh}$ or $\mathrm{PrP}^{\mathrm{PG} 14}-\mathrm{mCh}$

axons. f, Average net velocities of LAMP1-EGFP vesicles in $\mathrm{PrP}^{\mathrm{WT}}$-mCh or $\mathrm{PrP}^{\mathrm{PG} 14}-\mathrm{mCh}$

axons. $\mathrm{N}_{\text {tracks }}$ are shown inside bars. g, Percent time LAMP1-EGFP vesicles spent in

motion or paused in $\mathrm{PrP}^{\mathrm{WT}}$-mCh or $\mathrm{PrP}^{\mathrm{PG} 14}-\mathrm{mCh}$ axons. All values are shown as mean \pm SEM. ${ }^{* *} p<0.001,{ }^{* *} p<0.01,{ }^{*} p<0.05$, ns = not significant, Student's t-test $(\mathbf{e}-\mathbf{g})$, Tukey's correction (b). See also Supplementary Figure 9. 
a

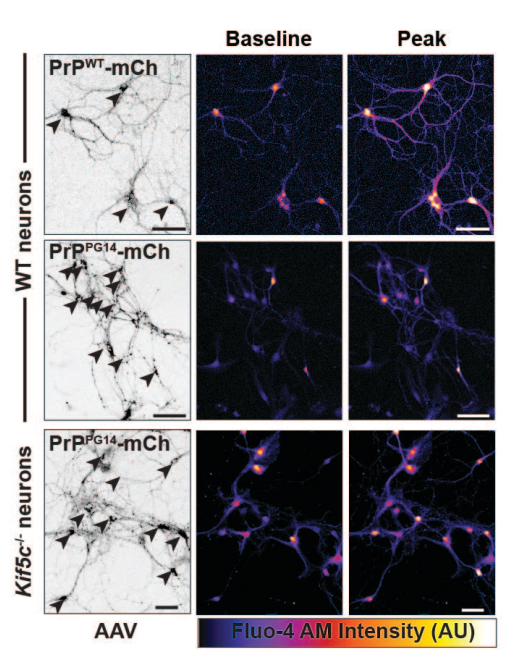

d

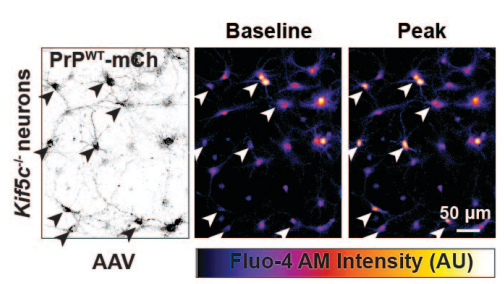

b
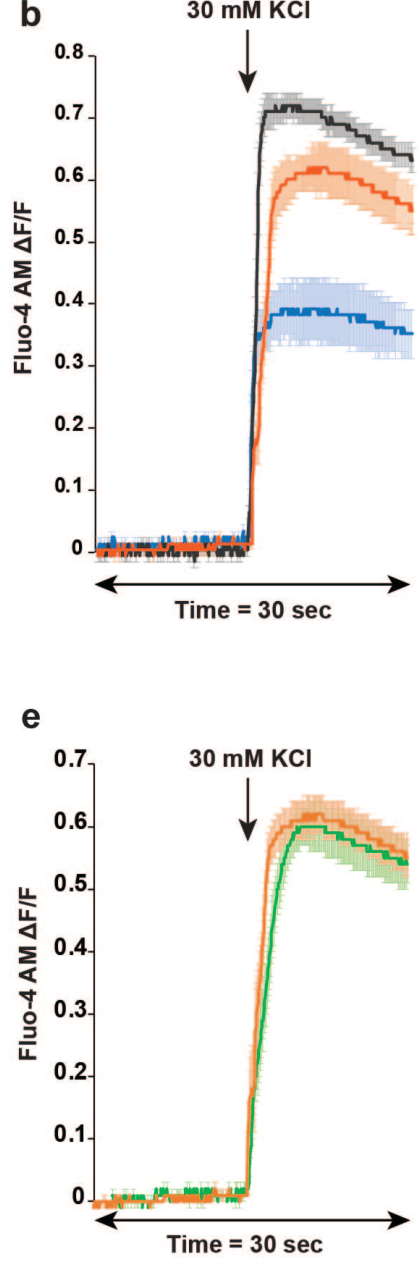

C

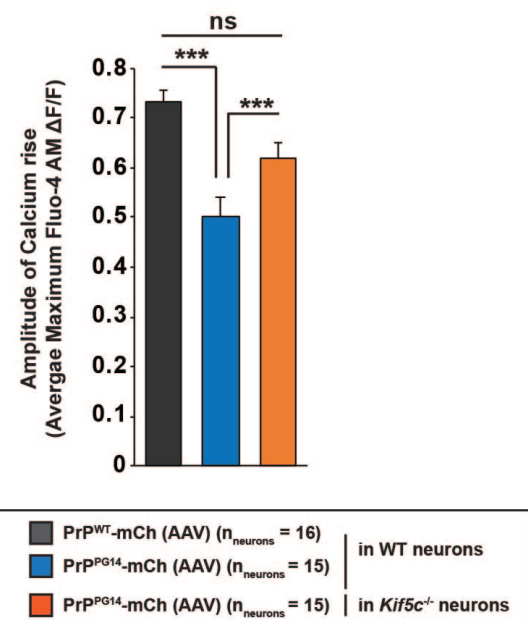

f

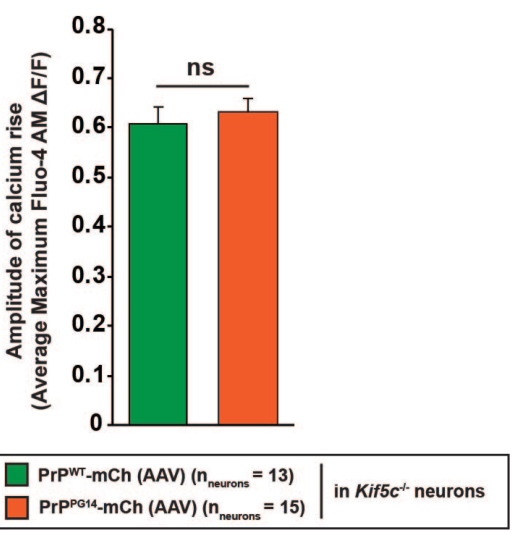

Figure 8. Kinesin-1-dependent PrP ${ }^{\mathrm{PG} 14}$ endoggresome formation impairs calcium influx dynamics. a, Representative images of $\mathrm{PrP}^{\mathrm{WT}}-\mathrm{mCh}$ or $\mathrm{PrP}^{\mathrm{PG} 14}-\mathrm{mCh} \mathrm{AAV}-$ transduced WT or Kif5c ${ }^{-/-}$neurons (arrowheads; left). Baseline and peak Fluo-4 AM intensities pre- and post-KCl treatment (middle, right). Scale bars $50 \mu \mathrm{m} . \mathbf{b}, \mathrm{KCl}$-induced depolarization response curve from normalized Fluo-4 AM intensities from (a). c, Average maximum amplitude of calcium rise from (b). d, Representative images of $\mathrm{PrP}^{\mathrm{WT}}$-mCh AAV-transduced $\mathrm{Kif5}^{-/-}$neurons (arrowheads). Baseline and peak Fluo-4 $\mathrm{AM}$ intensities pre- and post- $\mathrm{KCl}$ treatment (middle, right). e, $\mathrm{KCl}$-induced depolarization response curve from normalized Fluo-4 AM intensities from (d and a). f, Average maximum amplitude of calcium rise from (e). All values are shown as mean \pm SEM. 
bioRxiv preprint doi: https://doi.org/10.1101/2020.09.27.315846; this version posted September 28, 2020. The copyright holder for this preprint (which was not certified by peer review) is the author/funder. All rights reserved. No reuse allowed without permission.

${ }^{* * *} \mathrm{p}<0.001, \mathrm{~ns}=$ not significant, Student's t-test, Tukey's correction $(\mathbf{c}, \mathbf{f})$. See also

\section{Supplementary Figure 10.}




\section{Additional Information}

Endosomal Sorting in Neurons Drives the Biogenesis and Clearance of Mutant

\section{Prion Protein Aggregates}

Romain Chassefeyre, Tai Chaiamarit, Adriaan Verhelle, Sammy Weiser Novak, Leo R.

Andrade, Uri Manor, and Sandra E. Encalada 
Supplementary Movie 1. Degradation of PrP ${ }^{P G 14}-m C h$ in lysosomes in the soma.

Movie of a neuron co-expressing LAMP1-EGFP (green) and PrP ${ }^{\mathrm{PG} 14}-\mathrm{mCh}$ (red)

showing PrP ${ }^{\mathrm{PG} 14}$-mCh puncta disappearing. (Related to Supplementary Fig. 1g).

Supplementary Movie 2. Axonal transport of $\operatorname{PrP}^{\mathrm{WT}}-\mathrm{mCh}$ vesicles.

Inverted contrast movie showing axonal transport of $\mathrm{PrP}^{\mathrm{WT}}-\mathrm{mCh}$ vesicles in an axon of a cultured hippocampal neuron. (Related to Fig. 4 and Supplementary Fig. 4a).

Supplementary Movie 3. Axonal transport of PrP ${ }^{P G 14}-m C h$ vesicles and aggregates.

Inverted contrast movie showing axonal transport of $\mathrm{PrP}^{\mathrm{PG} 14}$-mCh vesicles and aggregates in an axon of a cultured hippocampal neuron. (Related to Fig. 4 and Supplementary Fig. 4a). 
a

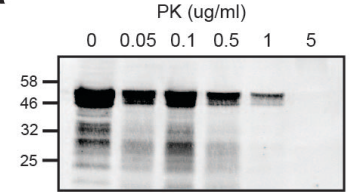

PrP'T-mCh

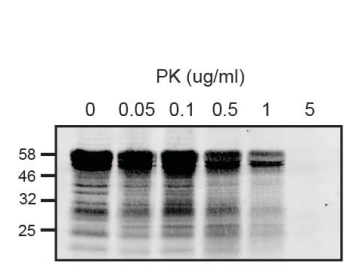

PrPPG14-mCh
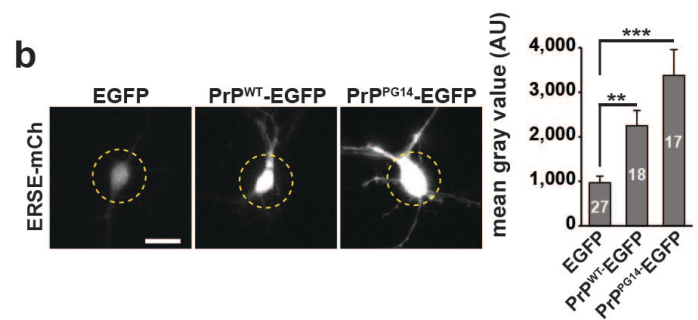

C
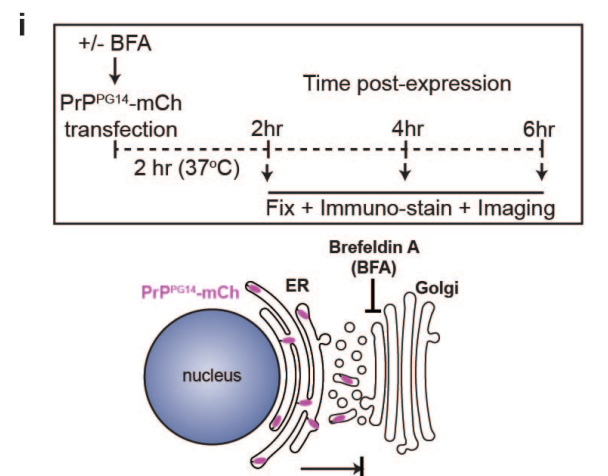

iii $1 / 48(2 \%)$

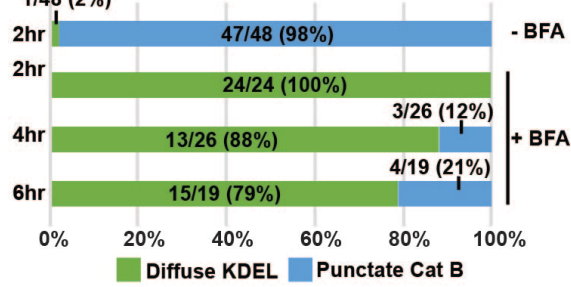

e
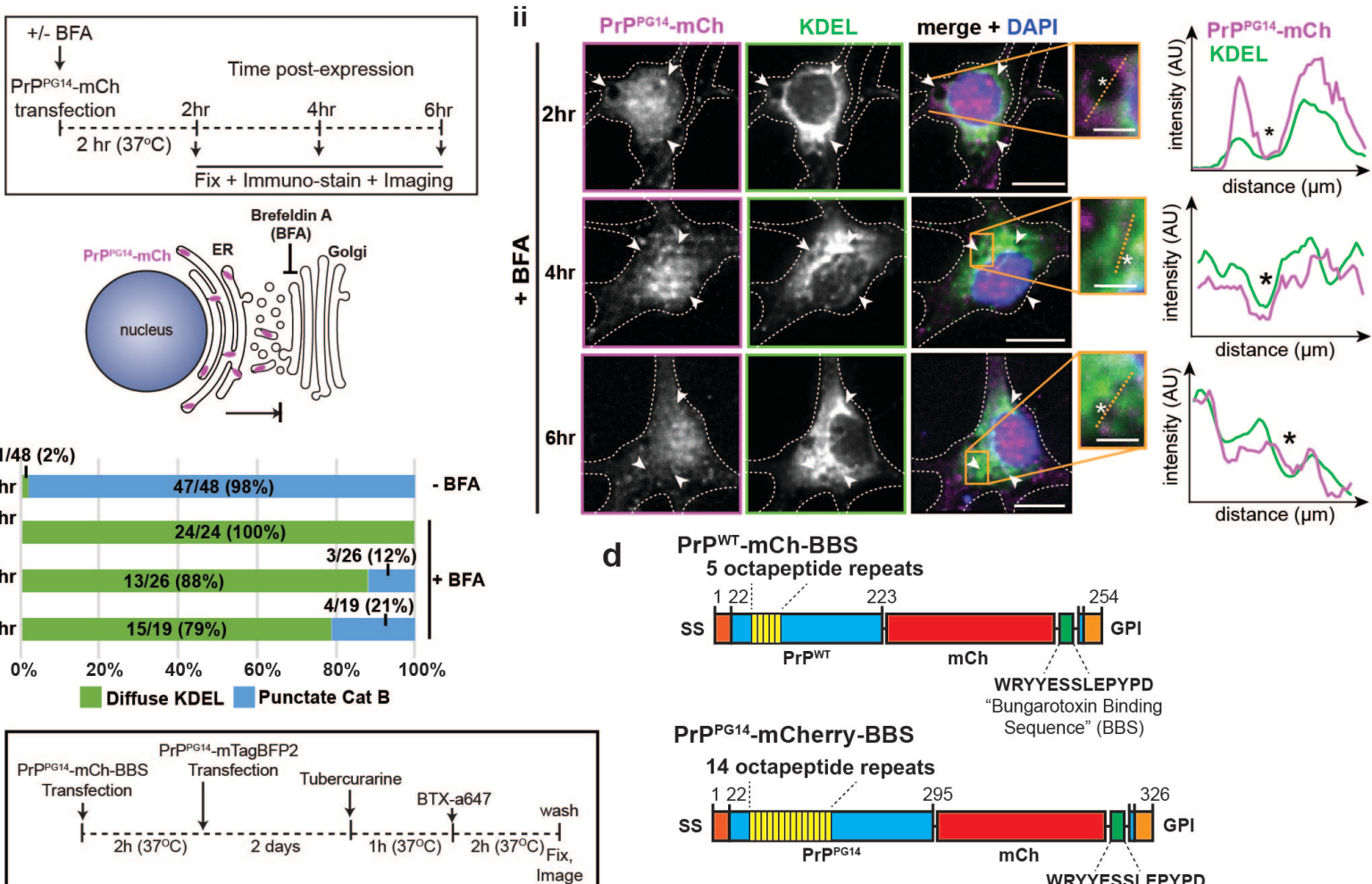

d PrPwt-mCh-BBS

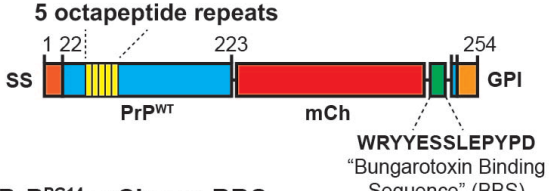

PrPPG14-mCherry-BBS

"Bequeton" (BBS)

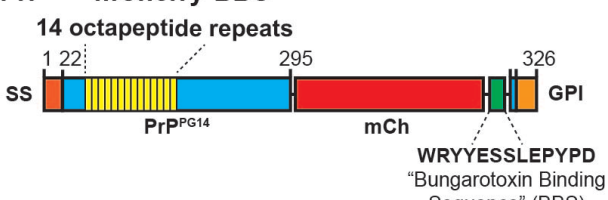

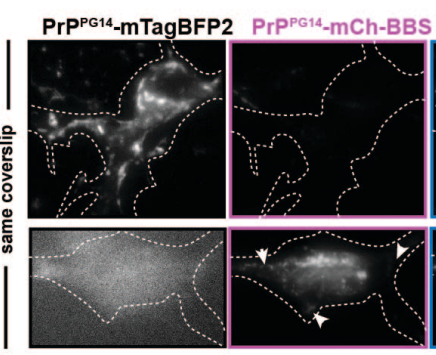

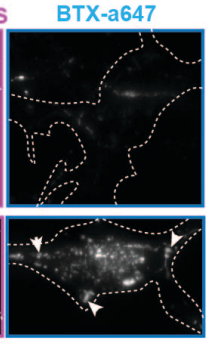

Merged

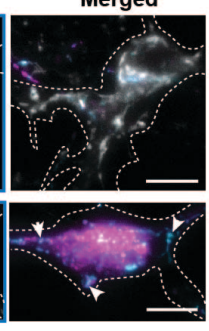

g

LAMP1-EGFP
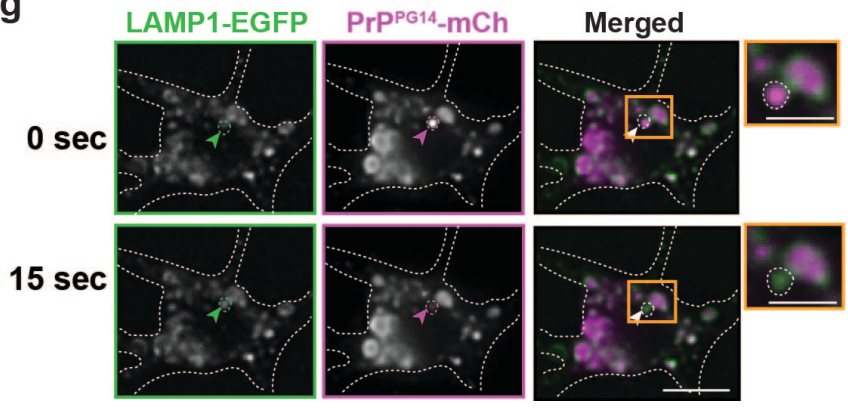

f

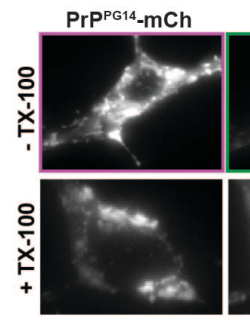

Bungarotoxin Binding

Sequence" (BBS)

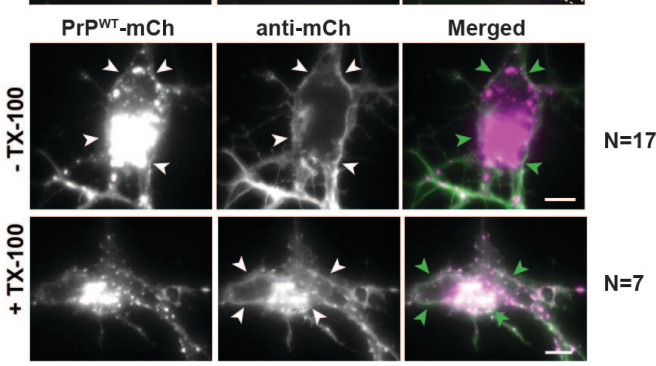

Chassefeyre, Chaiamarit, Verhelle et al. - 74 


\section{Supplementary Figure 1. PrP ${ }^{\mathrm{PG} 14}$ clearance Post-ER stress and cell surface export}

in neuronal soma. Related to Fig. 1. a, Representative Western blots showing of partial Proteinase K (PK) resistance by $\mathrm{PrP}^{\mathrm{PG} 14}$ aggregates from N2a cell lysates

$\left(\mathrm{N}_{\text {replicates }}=3\right) . \mathbf{b}$, Representative images and quantitation of neurons co-transfected with ERSE-mCh and EGFP (control), PrP ${ }^{W T}$-EGFP, or PrP ${ }^{P G 14}$-EGFP. Dotted lines indicate $30 \mu \mathrm{m}$ diameter circles used to measure total gray intensity values. Scale bar $=20 \mu \mathrm{m}$. Values are shown as mean \pm SEM. ${ }^{* *} p<0.001,{ }^{* *} p<0.01$, Student's t-test. $\mathrm{N}_{\text {cells }}$ are shown inside bars. c, (i) Experimental outline (top) and diagram (bottom) of BFA assay. (ii) Representative images of soma of neurons expressing PrP ${ }^{\mathrm{PG} 14}-\mathrm{mTagBFP} 2$ and stained with antibodies against ER (KDEL) or with DAPI nuclear marker at indicated time points in the presence of BFA. Arrowheads and asterisks point to colocalization or noncolocalization events, respectively, also shown in enlarged insets and linescan intensity profiles. Scale bars $=10 \mu \mathrm{m}$. Scale bars of insets $=250 \mathrm{~nm}$. (iii) Quantitation of PrP $\mathrm{P}^{\mathrm{PG} 14}$ mCh colocalization with KDEL or CatB from Fig. 1b. Numbers inside/above bars are total number and percentage of observed neurons. d, Schematic of PrP ${ }^{W T}-m C h-B B S$ and PrP ${ }^{P G 14}-m C h-B B S$ constructs. SS: signal sequence; GPI: GPI anchor. e, Experimental outline of BBS internalization assay (top). Representative images of two neurons taken from the same coverslip, sequentially transfected with $\mathrm{PrP}^{\mathrm{PG} 14}$-mCh-BBS and PrP ${ }^{\mathrm{PG} 14}$-mTagBFP2 constructs. Scale bars $=10 \mu \mathrm{m}$. f, Immunofluorescence images of axons expressing $\mathrm{PrP}^{\mathrm{PG} 14}-\mathrm{mCh}$ or $\mathrm{PrP}^{\mathrm{WT}}-\mathrm{mCh}$ in the presence or absence of detergent $(\mathrm{TX}-100)$. Scale bars $=10 \mu \mathrm{m} . \mathbf{g}$, Images of a neuron co-expressing LAMP1EGFP and $\mathrm{PrP}^{\mathrm{PG} 14}-\mathrm{mCh}$ at two time points. Scale bar $=10 \mu \mathrm{m}$. Arrowheads indicate a $\mathrm{PrP}^{\mathrm{PG} 14}-\mathrm{mCh}$ disappearing vesicle (enlarged in inset). Scale bars in insets $=5 \mu \mathrm{m}$. 
a

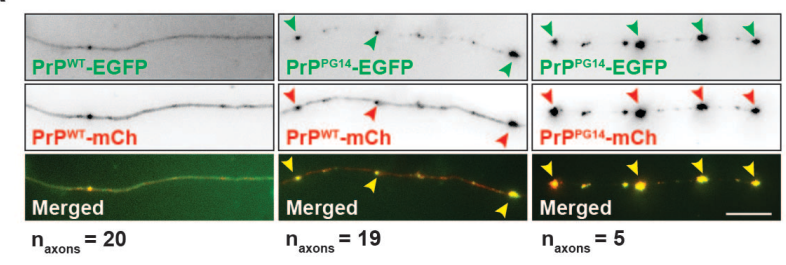

C

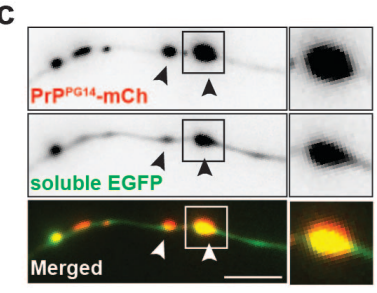

d

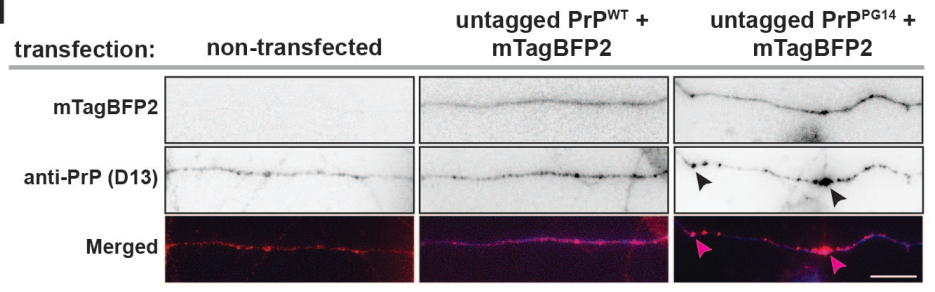

f

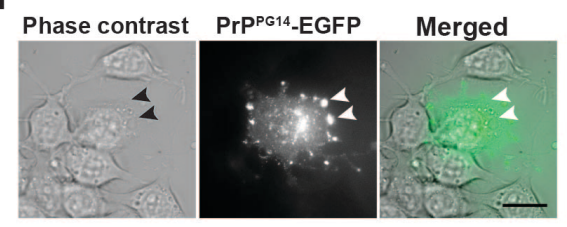

b
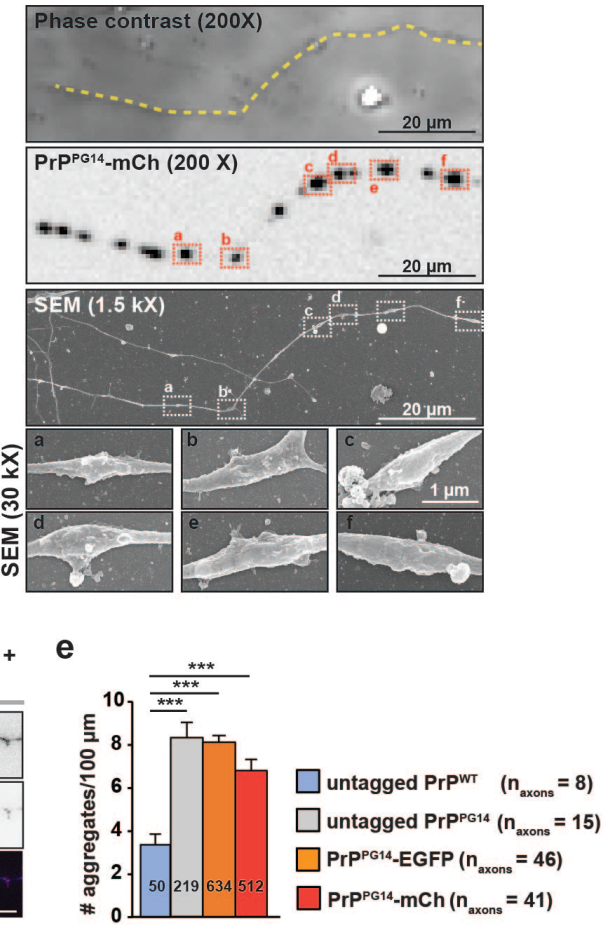

Supplementary Figure 2. PrP ${ }^{\mathrm{PG} 14}$ forms aggregates inside axons. Related to Fig. 2.

a, Images of axons from neurons co-transfected with PrPWT- EGFP plus PrP ${ }^{W T-m C h}$

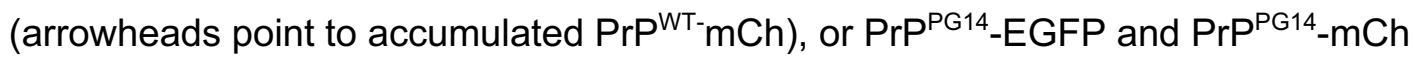
(arrowheads point to aggregating $\operatorname{PrP}^{\mathrm{PG} 14}$-EGFP). Scale bar $=10 \mu \mathrm{m}$. b, Correlative phase contrast (top), fluorescence (middle), and SEM (bottom) images of $\mathrm{PrP}^{\mathrm{PG} 14}-\mathrm{mCh}$ aggregate swellings. Insets (a-f) show enlargements of SEM boxes. $\mathrm{N}_{\text {swellings }}=20$. c, Images of axons co-expressing of $\mathrm{PrP}^{\mathrm{PG} 14}$-mCh and soluble EGFP (left panels). Scale bar $=5 \mu \mathrm{m}$. Insets of enlargements (right panels). Scale bar in insets $=2.5 \mu \mathrm{m}$.

Arrowheads point to aggregates. $\mathrm{N}_{\text {axons }}=39$. $\mathbf{d}$, Images of non-transfected axons, and those co-expressing untagged $\mathrm{PrP}^{\mathrm{WT}}$ or $\mathrm{PrP}^{\mathrm{PG} 14}$ with soluble mTagBFP2. Arrowheads 
point to aggregates. Scale bar $=10 \mu \mathrm{m}$. e, Quantification of aggregate densities from

(d). All values are shown as mean \pm SEM. ${ }^{* * *} p<0.001$, Kruskal- Wallis test. $N_{\text {aggregates }}$ are shown inside bars. f, Phase contrast and fluorescence images of a differentiated neuroblastoma (Neuro2a) cell expressing PrP ${ }^{P G 14}$-EGFP. Scale bar $=20 \mu \mathrm{m}$. 
a
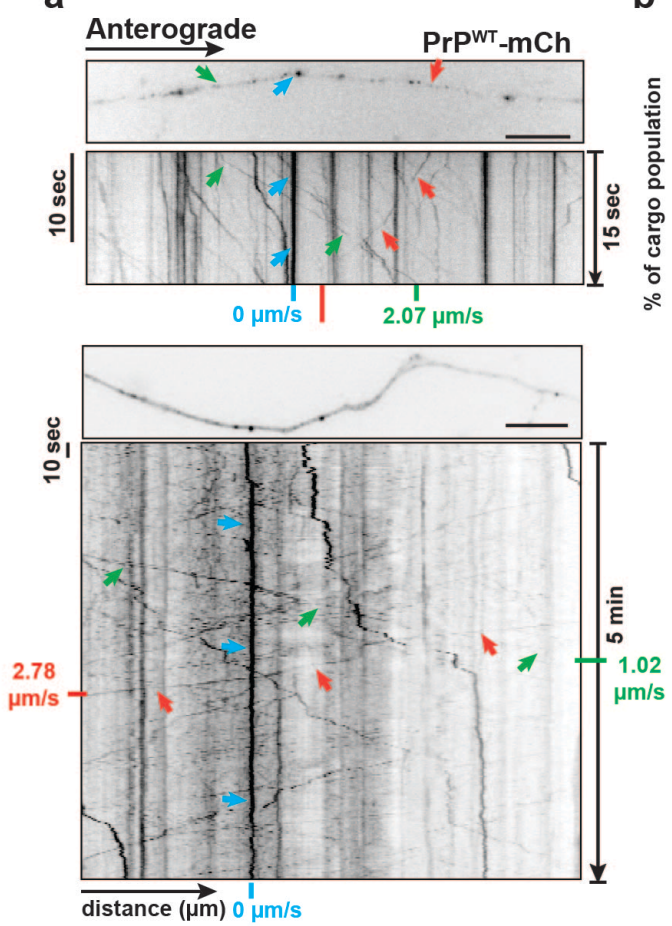

d

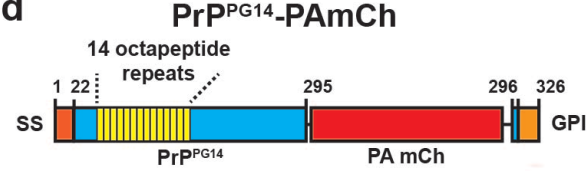

Before Photoactivation 1 min after Photoactivation
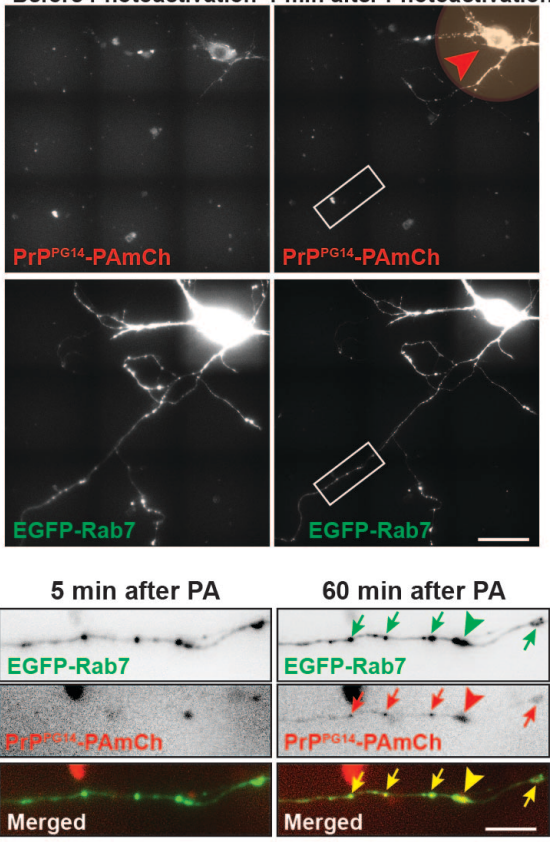

b
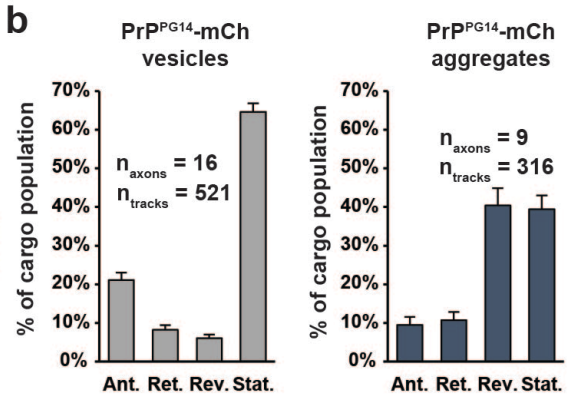

C

$\stackrel{\text { Anterograde }}{\longrightarrow}$

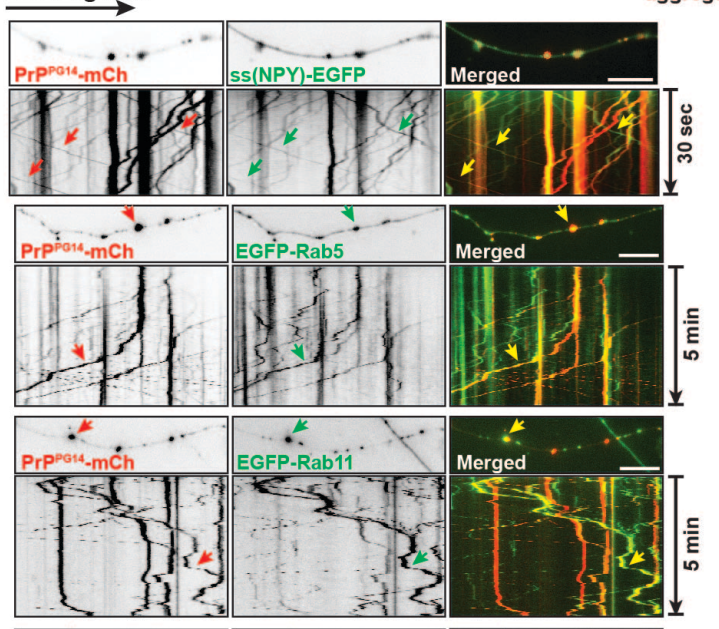

$\%$ PrPPG14-mCh vesicles and aggregates co-transported with:

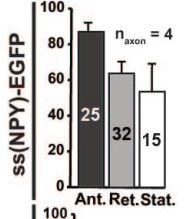

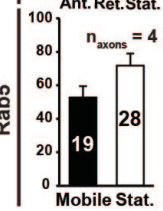
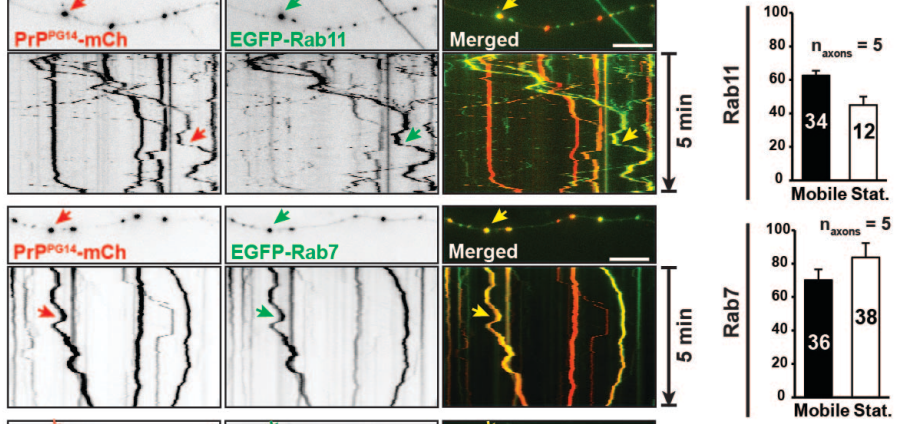

Prpana-men
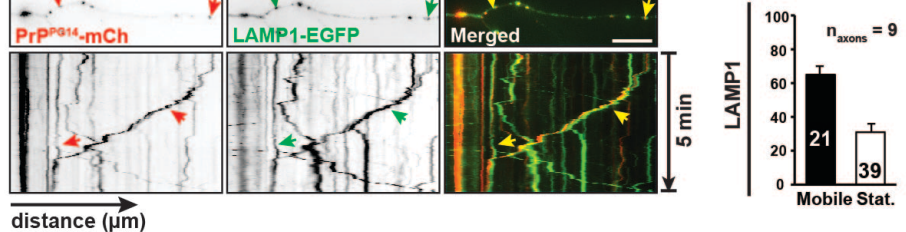

e
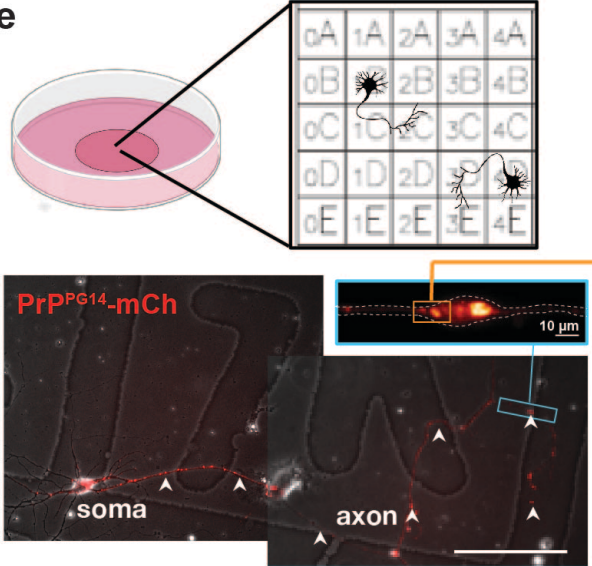

volumetric reconstruction aggregate site

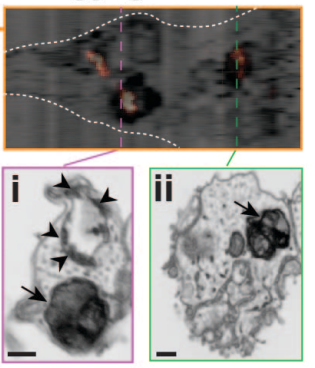




\section{Supplementary Figure 3. Post-Golgi PrP ${ }^{P G 14}$ vesicles sort to axonal endo-}

lysosomal compartments. Related to Fig. 3. a, First frames (top panels of each set) of time-lapse movies of axons expressing $\mathrm{PrP}^{\mathrm{WT}}-\mathrm{mCh}$, and corresponding kymographs (bottom panels of each set) at two time-scales. Arrows point to vesicles moving in the anterograde (green) or retrograde (red) directions, or that are stationary (blue). Scale bars $=10 \mu \mathrm{m} . \mathbf{b}$, Population breakdown of PrP ${ }^{\mathrm{PG} 14}-\mathrm{mCh}$ vesicles (left) and aggregates (right). All values are shown as mean \pm SEM. c, Left panels: Representative first-frame images and kymographs of time-lapse movies of axons co-expressing $\mathrm{PrP}^{\mathrm{PG} 14}$-mCh and various compartment markers: ss(NPY)-EGFP (Golgi-derived secretory), EGFP-Rab5 (early endosomes, EGFP-Rab11 (recycling endosomes), EGFP-Rab7 (LEs), or LAMP1EGFP (endo-lysosomes). Arrows point to cotransport. Right panels: Quantitation of cotransport between $\mathrm{PrP}^{\mathrm{PG} 14}-\mathrm{mCh}$ vesicles or aggregates and each of the respective markers. Ant. $=$ anterograde , Ret. $=$ retrograde, Stat. $=$ stationary. All values are shown as mean \pm SEM. $N_{\text {vesicles or aggregates }}$ are shown inside bars. Scale bars $=10 \mu \mathrm{m}$. $\mathbf{d}$, Top: schematic of PrP ${ }^{P G 14}$-PAmCh construct. SS: signal sequence; GPI: GPI anchor. Middle: representative images of a hippocampal neuron co-transfected with $\mathrm{PrP}^{\mathrm{PG} 14}$-PAmCh and EGFP-Rab7 before (left panels) and after (right panels) photoactivation (pink circle, arrowhead) of the cell body with a $405 \mathrm{~nm}$ laser. Inset shows axonal region enlarged in bottom panels. Scale bar of middle panels $=50 \mu \mathrm{m}$. Bottom panels: enlargements of inset in middle panels showing axonal PrP ${ }^{\mathrm{PG} 14}$-PAmCh and EGFP-Rab7 at indicated time points post-photoactivation. Arrows indicate colocalization. Arrowhead points to larger aggregate. $\mathrm{N}_{\text {neurons }}=3$. Scale bar of bottom panels $=10 \mu \mathrm{m}$. e, Top panels:

Schematic diagram of experimental setup showing neurons grown on gridded coverslips. Bottom panels: correlative fluorescence and SEM of neurons expressing $\mathrm{PrP}^{\mathrm{PG} 14}-\mathrm{mCh}$. White arrowheads point to axon. Scale bar $=100 \mu \mathrm{m}$. Top blue inset shows enlargement 
of blue rectangle. Right orange inset shows volumetric reconstruction. Magenta and green rectangles show single cross sections at two positions $(\mathbf{i}, \mathbf{i i})$ of the volumetric reconstruction. Arrowheads point to intralumenal vesicles and arrows point to aggregate structures inside membranous organelles. Scale bar of volumetric reconstruction and insets $=500 \mathrm{~nm}$. 


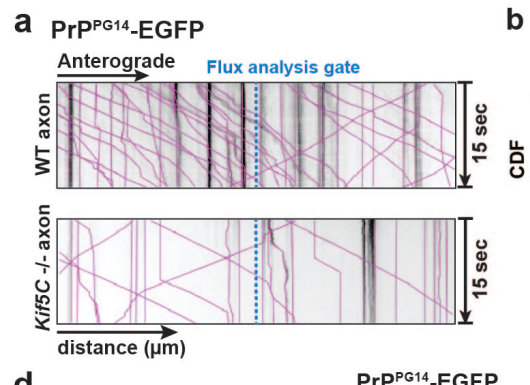

d
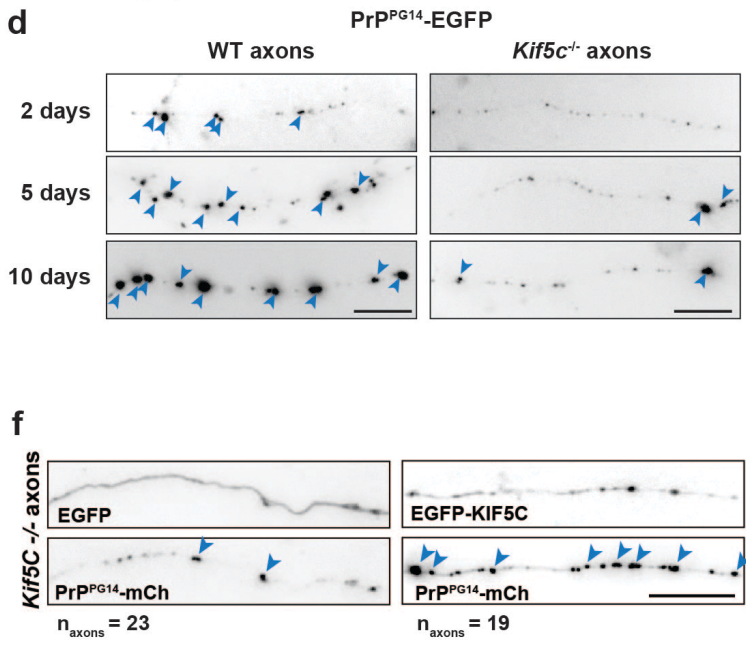

h

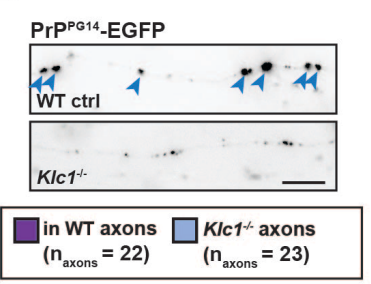

i

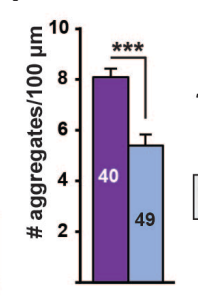

n

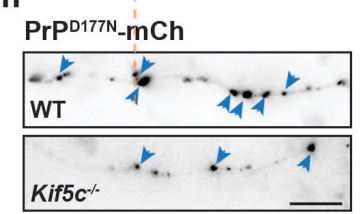

$\square W T \quad\left(n_{\text {axons }}=27\right)$
$\square K i f 5 c^{-1-} \quad\left(n_{\text {axons }}=25\right)$

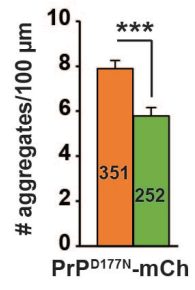

o b
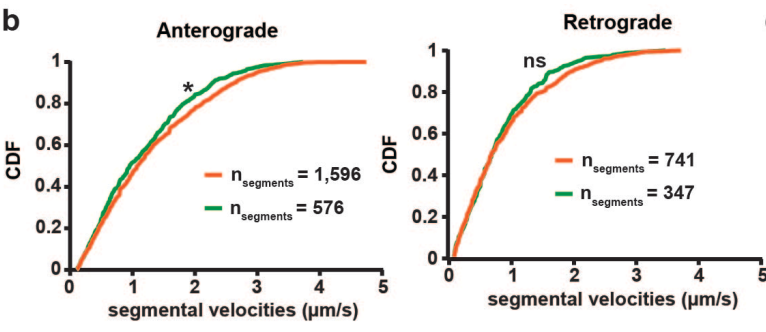

C 12 PrP ${ }^{\text {PG14-EGFP }}$

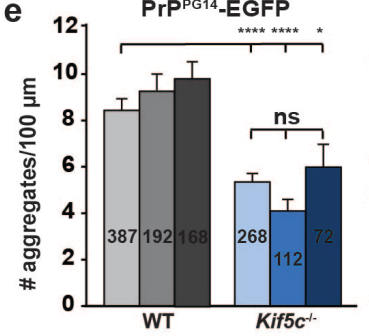

WT neurons
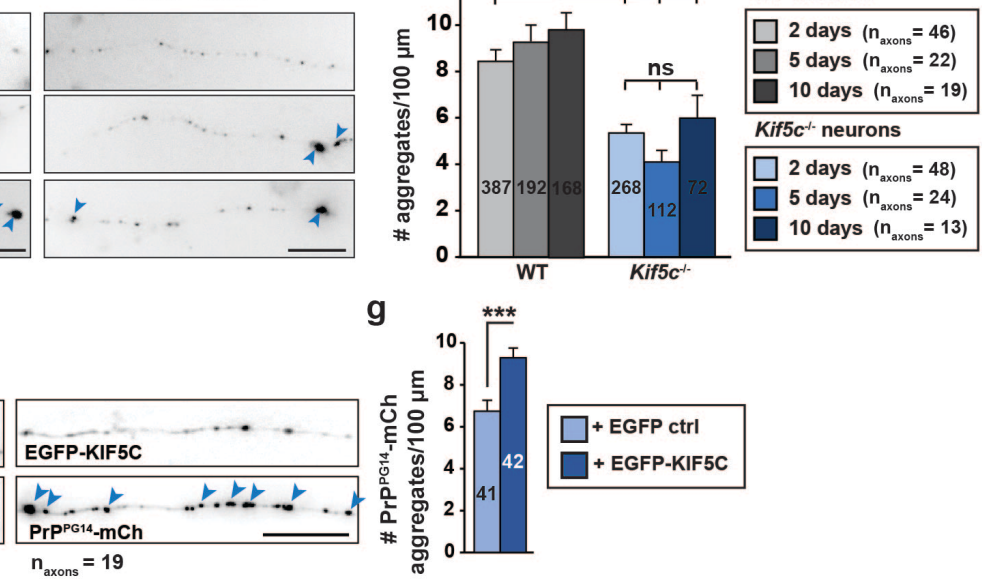

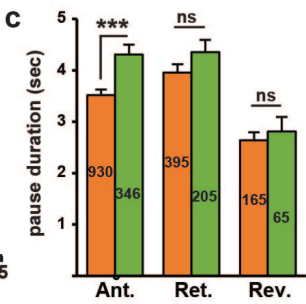

$\square$ in WT axons $\left(n_{\text {axons }}=59\right)$ $\square$ in Kif5 $c^{-/}$axons ( $\mathrm{n}_{\text {axons }}=48$ )

k kinesin-1B (Kif5b) conditional Ko PrPPG14-mCh
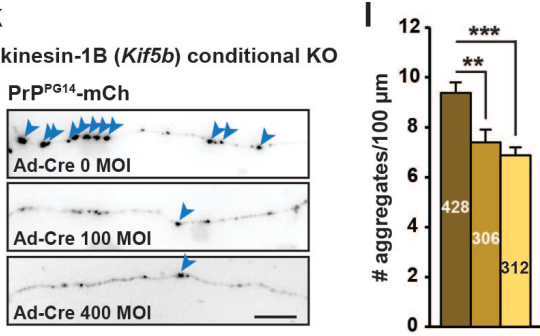

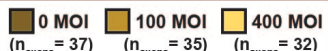

\section{Supplementary Figure 4. Kinesin-1- and DHC1-dependent PrP ${ }^{\mathrm{PG} 14}$ transport and}

aggregation in axons. Related to Fig. 4. a, Representative kymographs showing

position of gate (dotted blue line) used in flux analyses. $\mathbf{b}$, Cumulative distribution

frequency (CDF) of anterograde (top) and retrograde (bottom) segmental PrP ${ }^{\mathrm{PG} 14}$-EGFP 
vesicle velocities. ${ }^{*} p<0.05$, Kolmogorov-Smirnov test. c, Pause duration of anterograde, retrograde or reversing $\mathrm{PrP}^{\mathrm{PG} 14}$-EGFP vesicles. $\mathrm{N}_{\text {vesicles }}$ are shown inside bars. $\mathbf{d}$, Images of axons from WT (left) and Kif5c $c^{-/-}$(right) neurons expressing PrP ${ }^{P G 14}$-EGFP at various time points post-transfection. Arrowheads point to aggregates. Scale bars $=10 \mu \mathrm{m}$. e, Quantitation of aggregate densities from (d). f, Images of Kif5 $c^{-/-}$hippocampal neurons co-expressing soluble EGFP or EGFP-KIF5C and PrP ${ }^{P G 14}-\mathrm{mCh}$. Scale bar $=10 \mu \mathrm{m} . \mathbf{g}$, Quantitation of aggregate densities from (f). $\mathrm{N}_{\text {aggregates }}$ are shown inside bars. $\mathrm{N}_{\text {aggregates }}$ are shown inside bars. $\mathbf{h}$, Images of axons from WT (top) and $K / \mathrm{c} 1^{-/-}$(bottom) neurons expressing PrP ${ }^{P G 14}$-EGFP. Arrowheads point to aggregates. Scale bar $=10 \mu \mathrm{m}$. I, Quantitation of aggregate densities from (h). Naggregates are shown inside bars. j, DNA gel showing genotyping bands for Kinesin-1B hippocampal neuron extracts treated with 0 , 100 , or 400 multiplicity of infection (MOI) units of cre-recombinase adenovirus (AVV-cre) at 5 days post infection (dpi). The kineins-1B (Kif5B) II product is excised upon cretreatment to convert to a kinesin-1B I/+ product. $\mathrm{MOI}=$ Multiplicity of infection. $\mathbf{k}$, Images of axons from Kif5B conditional knockout neurons expressing $\mathrm{PrP}^{\mathrm{PG} 14}$-mCh at indicated AAV-cre units. Arrowheads point to aggregates. Scale bar $=10 \mu \mathrm{m}$. I, Quantitation of aggregate densities from (k). $\mathrm{N}_{\text {aggregates }}$ are shown inside bars. $\mathbf{m}$, Representative images of $\mathrm{PrP}^{\mathrm{D} 177 \mathrm{~N}_{-}}-\mathrm{mCh}$ aggregates in WT and $\mathrm{Kif5}^{-/-}$axons. Scale bar $=10 \mu \mathrm{m} . \mathbf{n}$, Quantitation of aggregate densities from (m). $\mathrm{N}_{\text {aggregates }}$ are shown inside bars. $\mathbf{0}$, Average net axonal velocities of PrP ${ }^{P G 14}$-EGFP vesicles in WT and DHC1 shRNA neurons. $\mathrm{N}_{\text {tracks }}$ are shown inside bars. Values in (c, e, $\left.\mathbf{g}, \mathbf{i}, \mathbf{I}, \mathbf{n}, \mathbf{0}\right)$ are shown as mean \pm SEM. ${ }^{*} p<0.05,{ }^{* *} p<0.01,{ }^{* * *} p<0.001,{ }^{* * *} p<0.0001$, Student's t-test and Kruskal- Wallis test. 
a
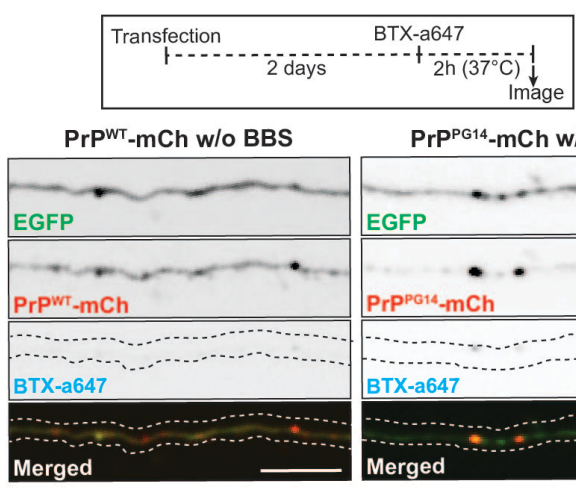

PrPPG14-mCh w/o BBS

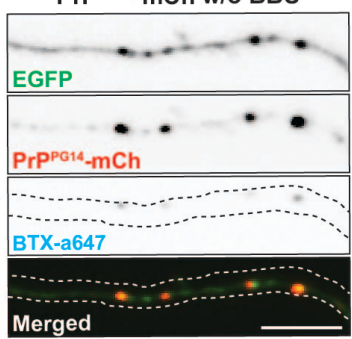

b

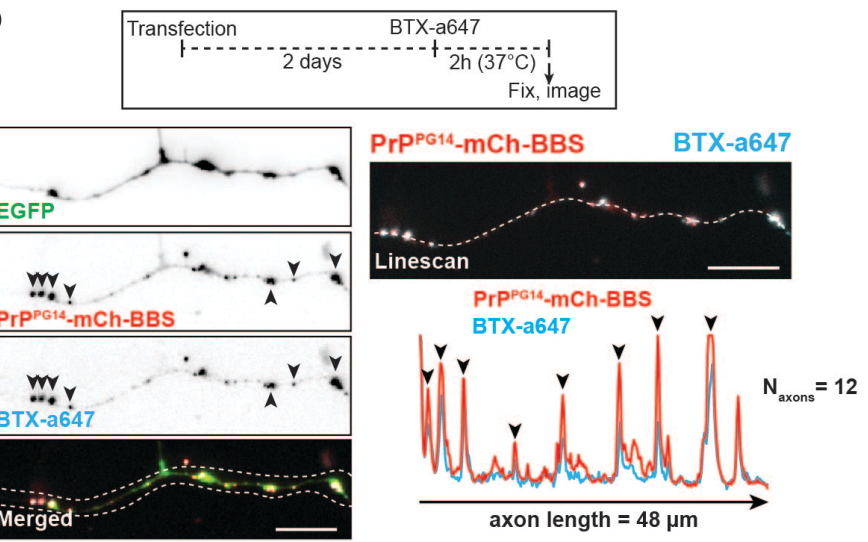

C

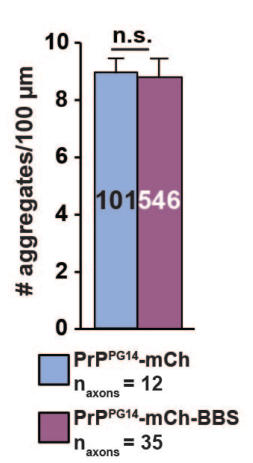

d
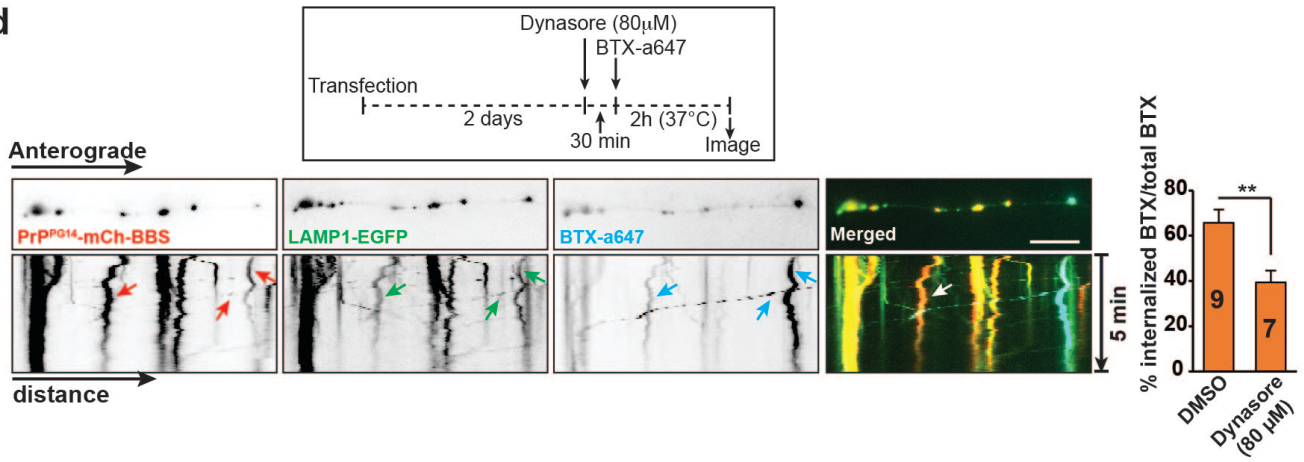

e

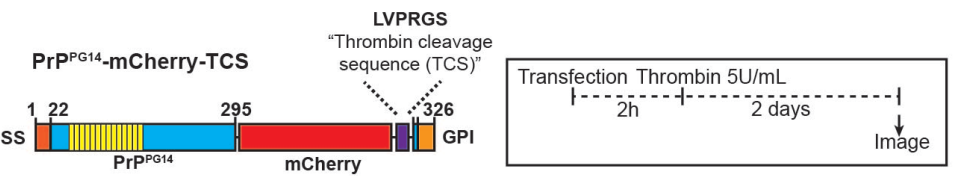

f

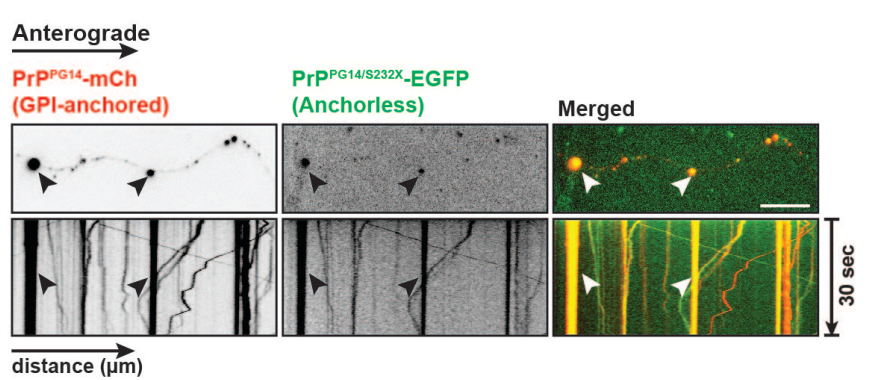

\section{Supplementary Figure 5. PrP ${ }^{\mathrm{PG} 14}$ transiently traffics to the cell surface before intra-}

axonal aggregation. Related to Fig. 5. a, Top: Experimental timeline of BTX-a647

labeling assay. Bottom: Representative images of neurons expressing $\mathrm{PrP}^{\mathrm{WT}}-\mathrm{mCh}$ or PrP ${ }^{\mathrm{PG} 14}-\mathrm{mCh}$, both without BBS sequence, after treatment with BTX-a647. Scale bars = $10 \mu \mathrm{m}$. b, Top: Experimental outline of BBS internalization and BTX-a647 labeling 
assay. Bottom: Representative images and corresponding line scan intensity profile of axons of neurons expressing soluble EGFP and PrP ${ }^{\mathrm{PG} 14}-\mathrm{mCh}-\mathrm{BBS}$ after BTX-a647 treatment. Arrowheads point to aggregates. Scale bars $=10 \mu \mathrm{m}$. c, Quantitation of aggregate densities from (a, b). $\mathrm{N}_{\text {aggregates }}$ are shown inside bars. $\mathbf{d}$, Top: Experimental outline of Dynasore endocytosis assay. Bottom left : Representative first-frame images of time-lapse movie, and kymographs of axons of neurons co-expressing PrP PG14 mCherry-BBS, LAMP1-EGFP and treated with BTX-a647. Arrows point to cotransporting tracks. Bottom right: quantitation of normalized percent internalized BTX-a647 signal. $\mathrm{N}_{\text {axons }}$ shown inside bars. Values are shown as mean \pm SEM. ${ }^{* *} p<0.01$, Student's t-test. Scale bar $=10 \mu \mathrm{m} . \mathbf{e}$, Left panel: schematic of PrPPG14-mCh-TCS construct. SS: signal sequence; GPI: GPI anchor. Right panel: Experimental outline of thrombin assay. f, Representative first-frame images of time-lapse movie, and kymographs of axons from neurons co-expressing GPI-anchored PrP ${ }^{\mathrm{PG} 14}$-mCh and anchorless PrP ${ }^{\mathrm{PG} 14 / \mathrm{S} 232 \mathrm{X}}$-EGFP. Arrowheads point to cotransport. $\mathrm{N}_{\text {axons }}=7$. Scale bar $=10 \mu \mathrm{m}$. 

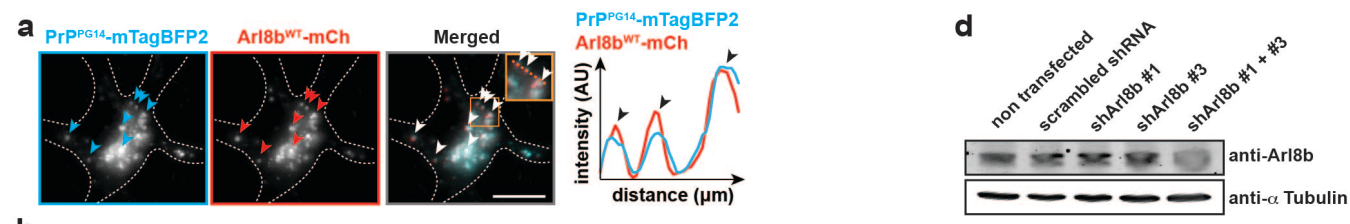

b Anterograde

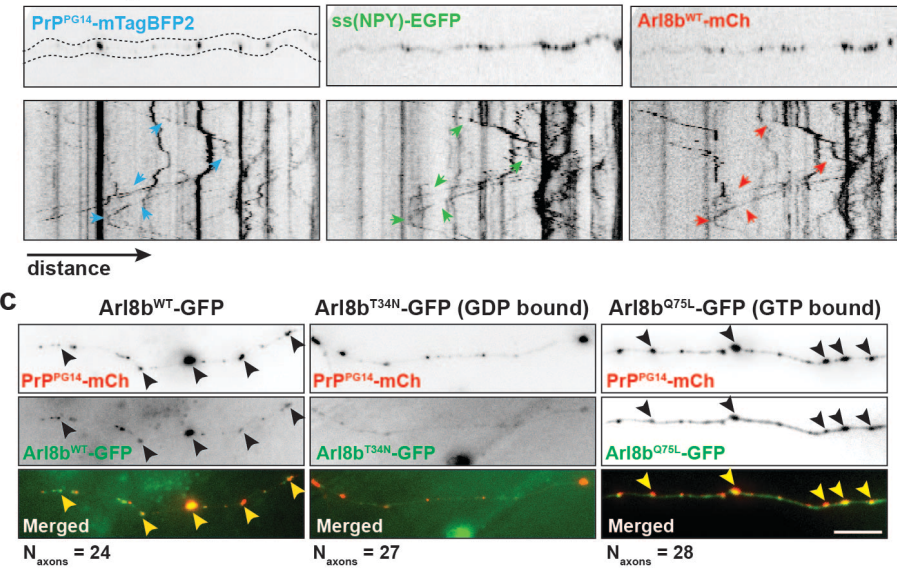

\section{Merged}

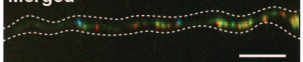



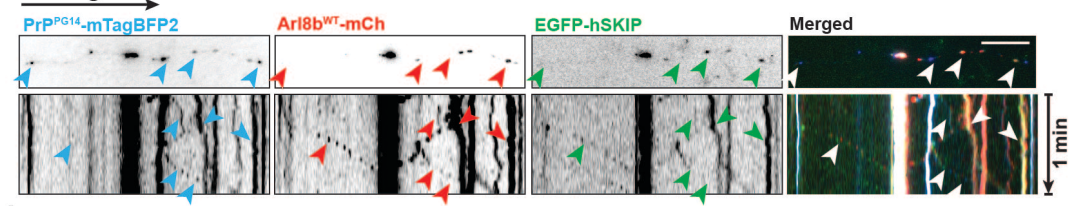

h

$\underset{\text { Anterograde }}{\longrightarrow}$

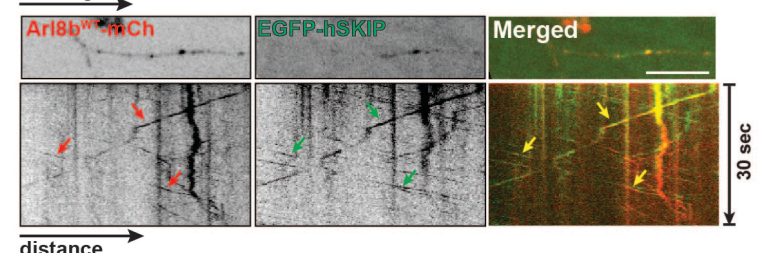

i Anterograde

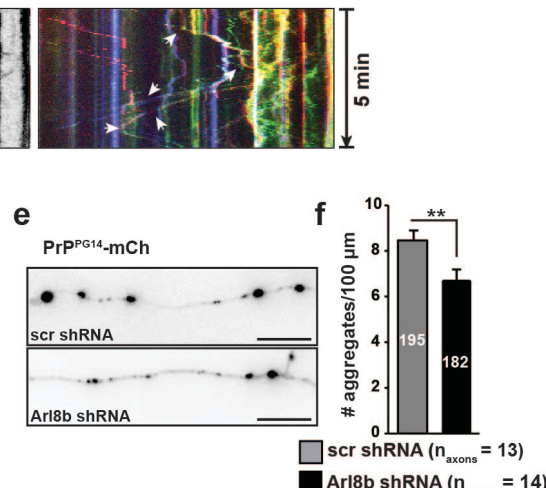

Arl8b shRNA $\left(n_{\text {axons }}=14\right)$

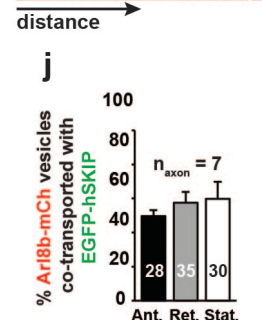

k

I
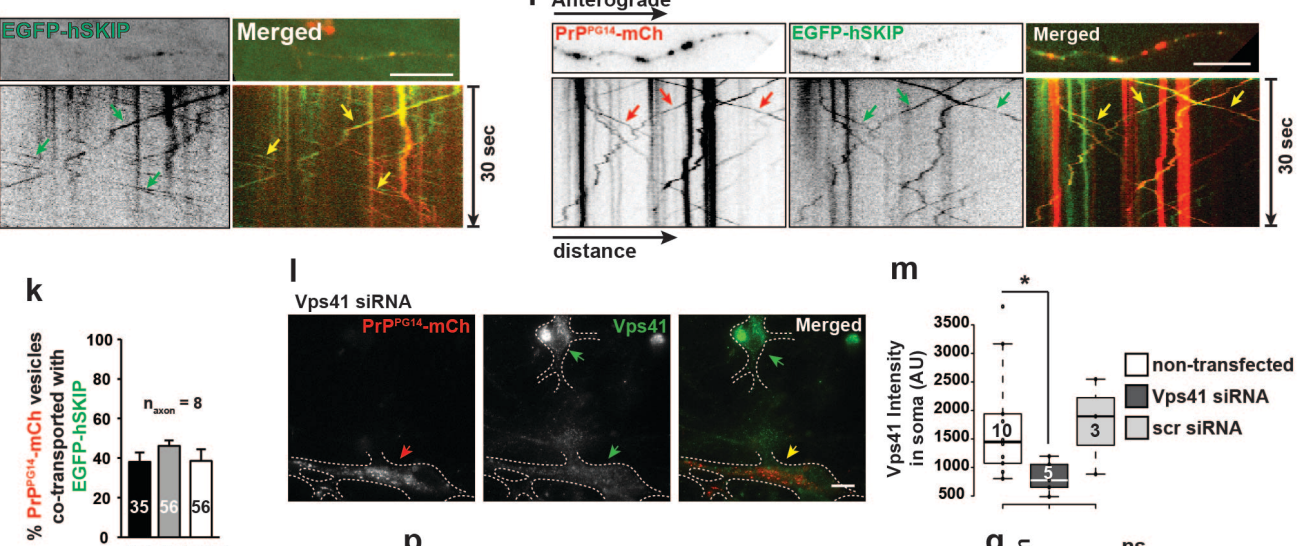

m
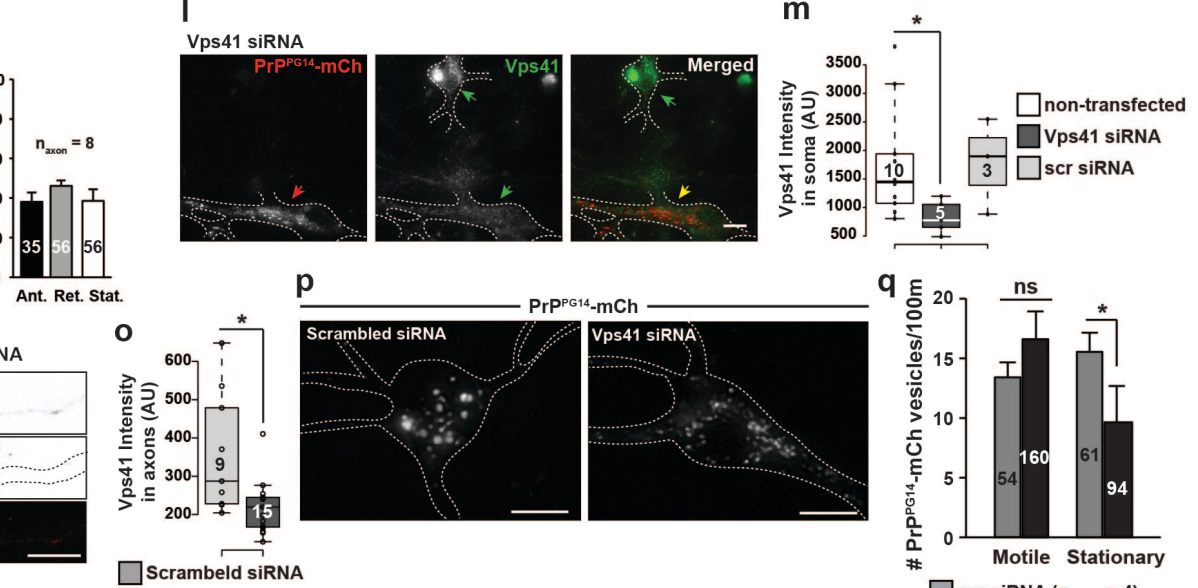

p

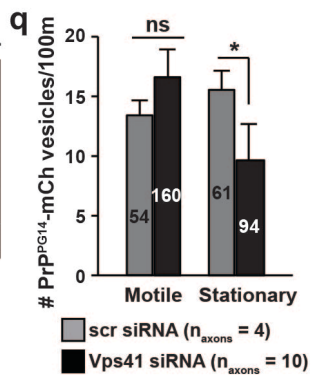




\section{Supplementary Figure 6. Arl8b recruits the HOPS complex to $\mathrm{PrP}^{\mathrm{PG} 14}$ vesicles to} promote fusion, and aggregation. Related to Fig. 6. a, Left panels: representative images of hippocampal soma co-expressing PrP ${ }^{\mathrm{PG} 14}-\mathrm{mTagBFP}$ and $\mathrm{Arl} 8 \mathrm{~b}^{\mathrm{WT}}-\mathrm{mCh}$. Arrowheads point to colocalization. Right pane: line scan intensity profiles of region indicated by dotted orange line inside inset (right). Scale bar $=10 \mu \mathrm{m}$. b, Representative first-frame images of time-lapse movies, and corresponding kymographs of axons from neurons co-expressing PrP ${ }^{\mathrm{PG} 14}$-mTagBFP2, ss(NYP)-EGFP and Arl8b ${ }^{\mathrm{WT}}-\mathrm{mCh}$. Arrows point to cotransport. $\mathrm{N}_{\text {axons }}=4$. Scale bar $=10 \mu \mathrm{m}$. c, Representative images of neurons

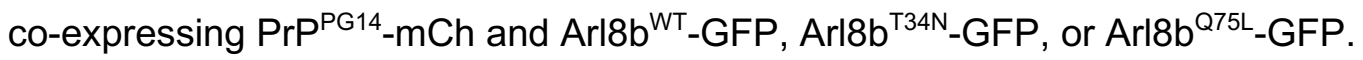
Arrowheads point to colocalization. Scale bar $=10 \mu \mathrm{m}$. d, Representative Western blot of N2a cell lysates treated with indicated shRNAs. $\mathrm{N}_{\text {replicates }}=3$. e, Representative images of axons from neurons expressing $\mathrm{PrP}^{\mathrm{PG} 14}$-mCh treated with scrambled or Arl8b shRNAs. Scale bars $=10 \mu \mathrm{m}$. f, Quantitation of aggregate densities from (e). $\mathrm{N}_{\text {aggregates }}$ are shown inside bars. g, Representative first-frame images of time-lapse movies, and corresponding kymographs of axons from neurons co-expressing PrP ${ }^{P G 14}-m T a g B F P 2$, Arl8b $b^{\text {WT }}-m$ Ch and EGFP-hSKIP. Arrowheads point to cotransport. $\mathrm{N}_{\mathrm{axons}}=15$. Scale bar $=10 \mu \mathrm{m} . \mathbf{h}$, Representative first-frame images of time-lapse movies, and corresponding kymographs of axons from neurons co-expressing Arl8b ${ }^{\mathrm{WT}}-\mathrm{mCh}$ and EGFP-hSKIP. Arrows point to cotransport. Scale bar $=10 \mu \mathrm{m}$. i, Representative first-frame images of time-lapse movies, and corresponding kymographs of axons from neurons coexpressing PrP ${ }^{P G 14}-m C h$ and EGFP-hSKIP. Arrows point to cotransport. Scale bar $=10$ $\mu \mathrm{m}$. j, Quantitation of percentage of cotransporting Arl8b $\mathrm{b}^{\mathrm{WT}}-\mathrm{mCh}$ and EGFP-hSKIP vesicles from $(\mathbf{H}) . \mathrm{N}_{\text {vesicles }}$ are shown inside bars. $\mathbf{k}$, Quantitation of percentage of cotransporting PrP ${ }^{P G 14}$-mCh and EGFP-hSKIP vesicles from (i). $\mathrm{N}_{\text {vesicles }}$ are shown inside bars. I, Immunofluorescence images of the soma of neurons expressing PrP ${ }^{P G 14}-m C h$, 
transfected with Vps41 siRNA, and stained with antibodies against Vps41. Arrows point to two neurons that either were transfected (top) or untransfected (bottom) with Vps41 shRNA. Scale bar $=10 \mu \mathrm{m} . \mathbf{m}$, Quantitation of Vps41 signal intensity from (I). Box shows lower (Q1) and upper (Q3) quartile and median. Whiskers mark the 9 to 91 percentile range. Datapoints outside of this range are represented by individual dots. $\mathrm{N}_{\text {cells }}$ are shown inside boxplots. $\mathbf{n}$, Representative axons from neurons co-transfected with PrP ${ }^{P G 14}$-mCh and Vps41 siRNA, stained with antibodies against Vps41. Arrowheads indicate aggregate. Scale bars $=10 \mu \mathrm{m}$. o, Quantitation of Vps41 signal intensity from (n). Box shows lower (Q1) and upper (Q3) quartile and median. Whiskers mark the 9 to 91 percentile range. Datapoints outside of this range are represented by individual dots. $\mathrm{N}_{\text {axons }}$ are shown inside boxplots. $\mathbf{p}$, Representative images of soma of neurons cotransfected with PrP ${ }^{P G 14}-m C h$ and shRNA scrambled or Vps41 siRNA. Scale bars $=10$ $\mu \mathrm{m}$. q, Quantitation of motile and stationary axonal PrP ${ }^{\mathrm{PG} 14}-\mathrm{mCh}$ vesicle densities in neurons co-transfected with scrambled or Vps41 shRNAs. $N_{\text {vesicles }}$ are shown inside bars. All values in $(\mathbf{f}, \mathbf{j}, \mathbf{k}, \mathbf{q})$ are shown as mean \pm SEM. Values in $(\mathbf{m}, \mathbf{0})$ are shown as Tukey's box plot. ${ }^{*} p<0.05,{ }^{* *} p<0.01$, ns = non-significant. Student's t-test $(\mathbf{f}, \mathbf{q})$, Wilconxon rank-sum test $(\mathbf{m}, \mathbf{0})$. 
a

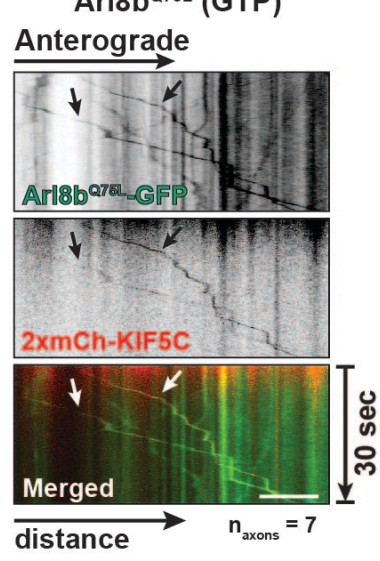

C

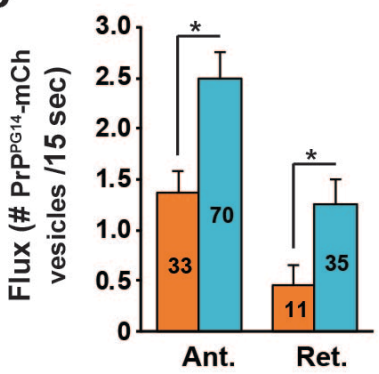

e

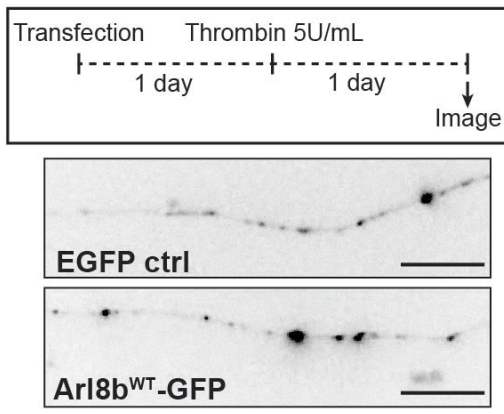

PrP ${ }^{\mathrm{PG} 14}$-mCh-TCS

(treated $\mathrm{w} /$ Thrombin $5 \mathrm{U} / \mathrm{ml}$ )
$\operatorname{Arl}^{18 b^{\top 34 N}}(\mathrm{GDP})$

Anterograde

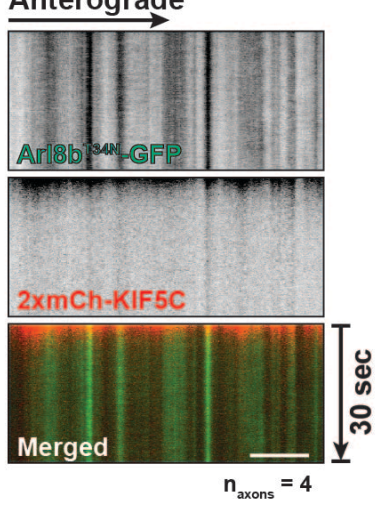

b

$\underset{\text { Anterograde }}{\stackrel{(\cdot 2}{\longrightarrow}}$

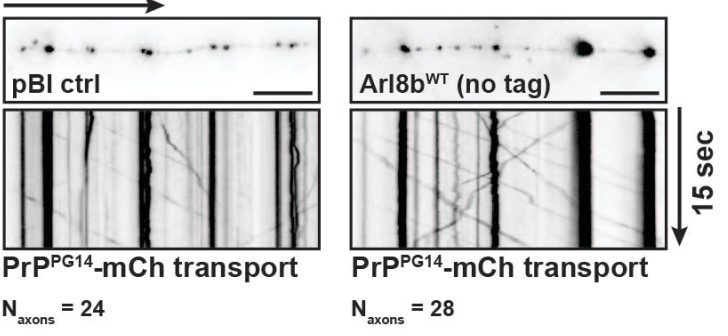

d

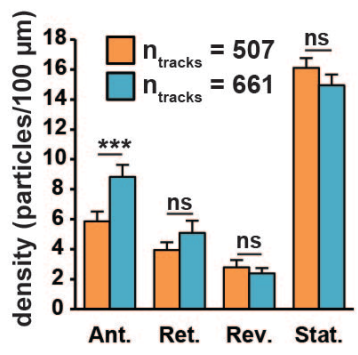

f

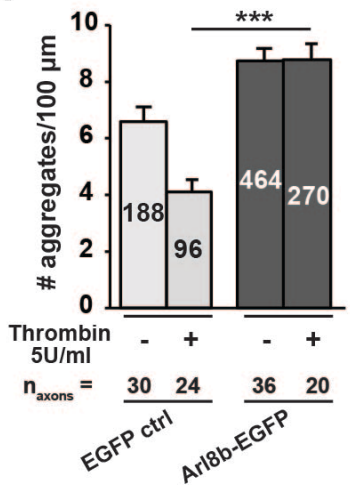

\section{Supplementary Figure 7. Direct and indirect ARESTA act in parallel and converge}

on Arl8b. Related to Fig. 6. a, Representative kymographs of axons co-expressing

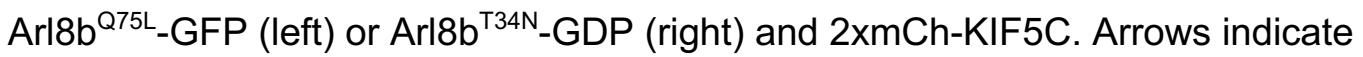
cotransport. Scale bars $=10 \mu \mathrm{m} . \mathbf{b}$, Representative first-frame images of time-lapse movies, and corresponding kymographs of axons from neurons expressing $\mathrm{PrP}^{\mathrm{PG} 14}-\mathrm{mCh}$ and transfected with empty $\mathrm{pBi}$ vector (control) or untagged Arl8b $\mathrm{b}^{\mathrm{WT}}$. Scale bars $=10$ $\mu$ m. c, Quantitation of PrPPG14-EGFP vesicle flux from (b). $\mathrm{N}_{\text {vesicles }}$ are shown inside bars. 
d, Quantitation of vesicle densities from (b). e, Top: Experimental outline of thrombin assay. Bottom: Representative images of axons of neurons co-expressing $\mathrm{PrP}^{\mathrm{PG} 14}-\mathrm{mCh}-$ TCS a. d soluble EGFP or Arl8b ${ }^{W T}$-GFP. Scale bar $=10 \mu \mathrm{m}$. f, Quantitation of PrP PG14_ mCh aggregate densities from (e). $\mathrm{N}_{\text {aggregates }}$ are shown inside bars. Ant. = anterograde; Ret. $=$ retrograde; Rev. $=$ reversal; Stat. $=$ stationary. Values in $(\mathbf{c}, \mathbf{d}, \mathbf{f})$ are shown as mean \pm SEM. ${ }^{*} p<0.05,{ }^{* * *} p<0.001$, Kruskal- Wallis test. 
a
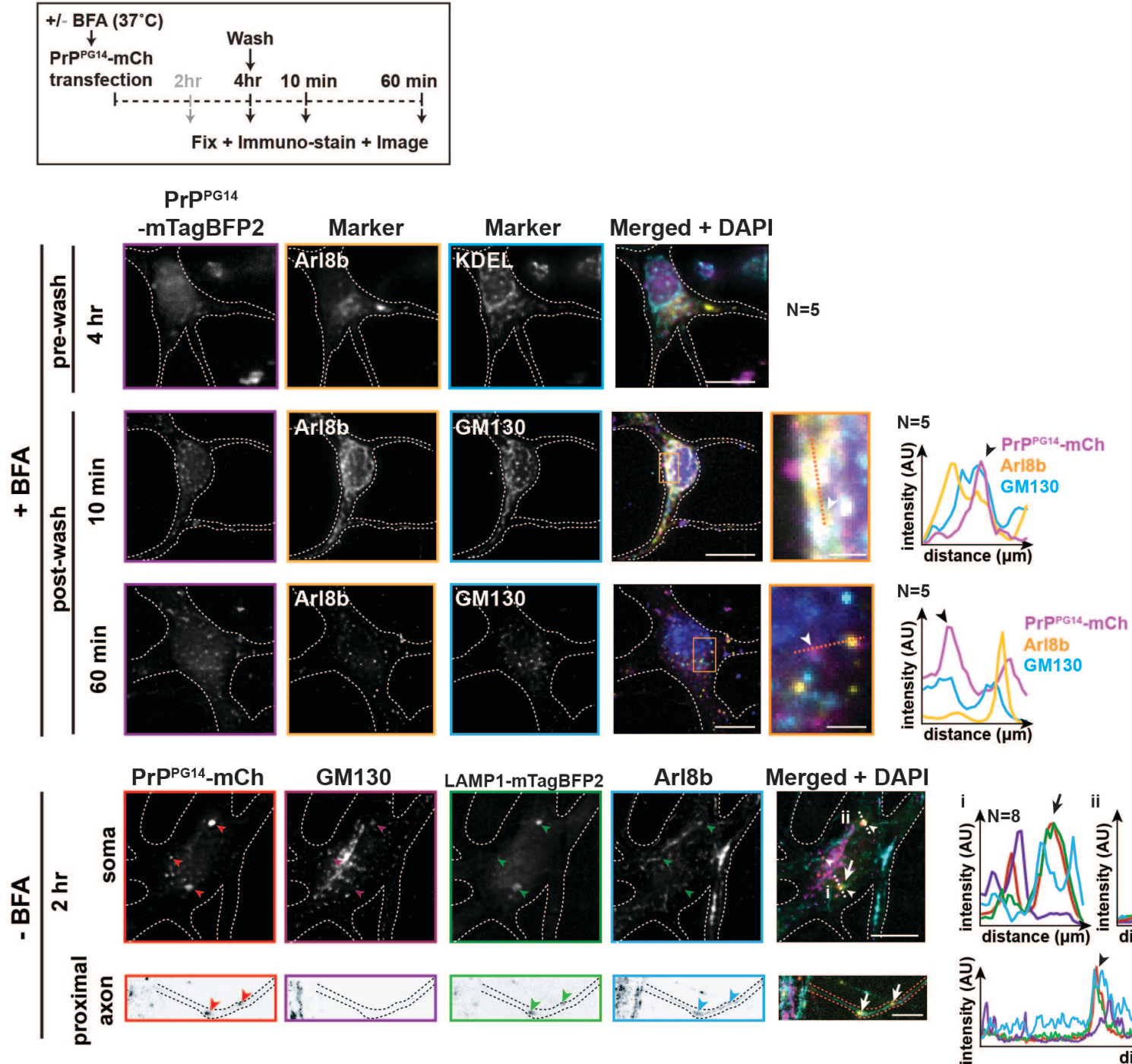

b
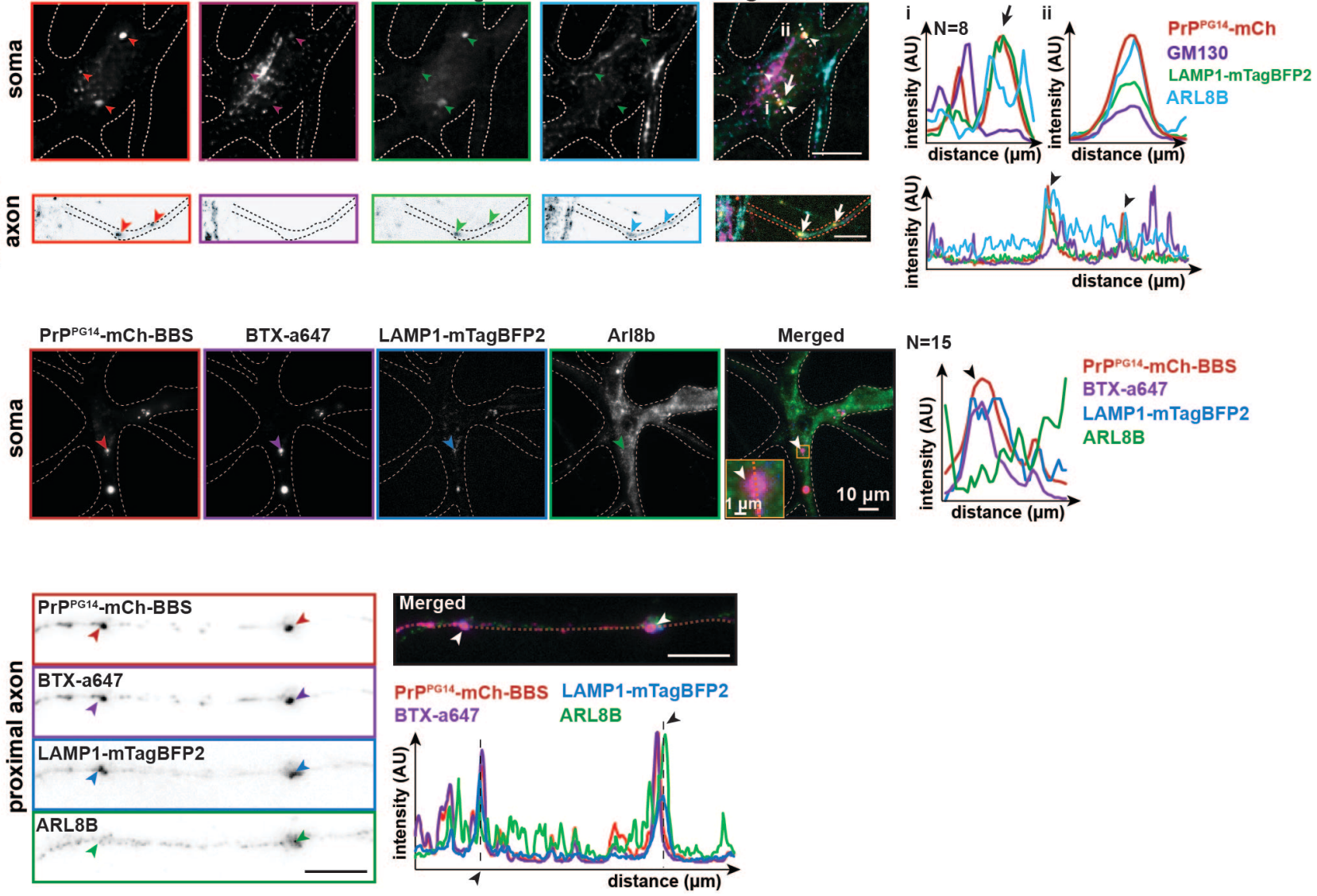

Chassefeyre, Chaiamarit, Verhelle et al. - 90 
Supplementary Figure 8. Arl8b is a key determinant of PrP ${ }^{P G 14}$ axonal entry. a, Top panels : experimental outline of BFA assay. Bottom panels: representative images of soma and proximal axons of different neurons expressing PrPPG14_ mTagBFP2 or PrPPG14-mCh + Lamp1-mTagBFP2 and stained with antibodies against ARL8B, ER (KDEL), Golgi (GM130), or with DAPI nuclear marker at indicated time points and conditions. Graphs to the right are of line scans of dotted lines inside insets. Arrowheads point to colocalization events, also shown in enlarged insets and in linescan intensity profiles. Scale bars in main panels = $10 \mu \mathrm{m}$. Scale bars in insets $=500 \mathrm{~nm} . \mathbf{b}$, Representative images PrPPG14 $-\mathrm{mCh}-$ BBS internalization assay of the soma (top panels) and axons (bottom panels), of neurons co-transfected with PrPPG14-mCh-BBS and LAMP1-mTagBFP2, labeled with BTX-a647, and stained with an antibody against ARL8B. Arrowheads point to colocalization events, also shown in enlarged insets and in linescan intensity profiles. Scale bars in main panels $=10 \mu \mathrm{m}$. Scale bars in inset $=1 \mu \mathrm{m}$. 
a

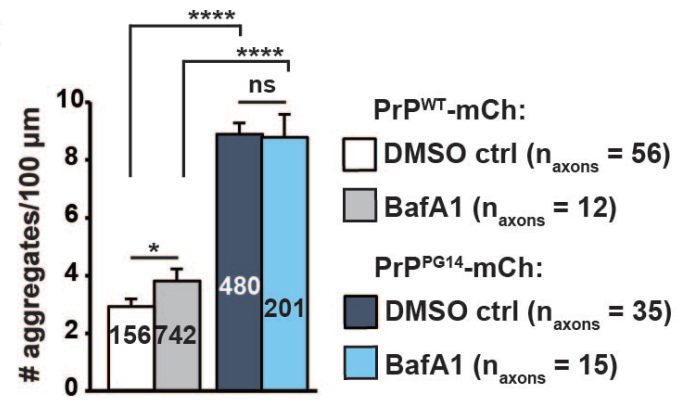

b

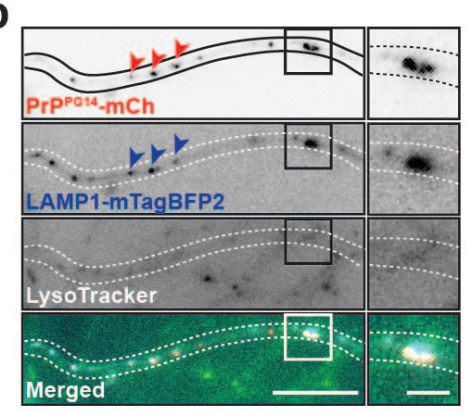

C

Anterograde

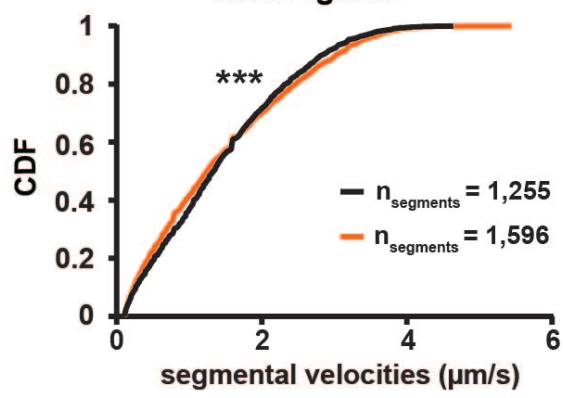

Retrograde

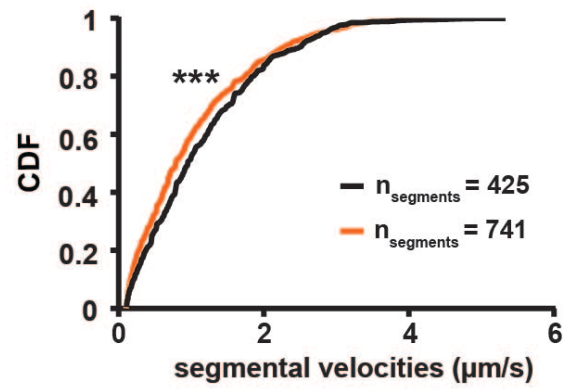

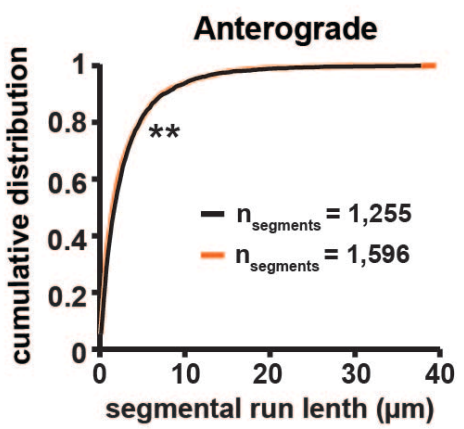

Retrograde

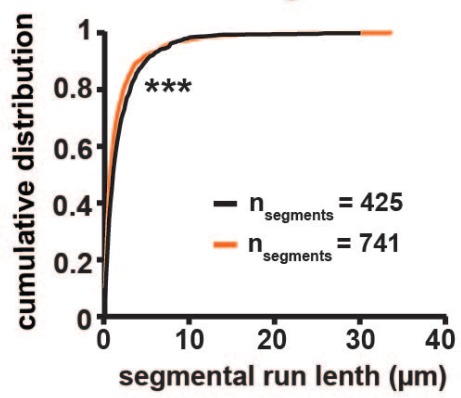

- PrPWT-EGFP vesicles $\left(\mathrm{n}_{\text {axons }}=42\right)$

- PrPPG14-EGFP vesicles $\left(n_{\text {axons }}=59\right)$

Supplementary Figure 9. Lysosomal degradation impairments and retrograde transport deficits in PrP ${ }^{P G 14}$-expressing axons. Related to Figure 7. a, Quantitation of axonal $\mathrm{PrP}^{\mathrm{PG} 14}-\mathrm{mCh}$ aggregate densities in neurons treated with BafA1. $\mathrm{N}_{\text {aggregates }}$ are shown inside bars. b, Representative images of axons of neurons co-expressing PrP ${ }^{P G 14}-m C h$ and LAMP1-mTagBFP2 and treated with LysoTracker. Arrowheads point to colocalization. Right panels are enlargements of boxed regions. $\mathrm{N}_{\text {axons }}=21$. Scale bar of main figure $=10 \mu \mathrm{m}$. Scale bar of inset $=2.5 \mu \mathrm{m}$. c , Cumulative distribution frequencies (CDFs) of anterograde (top) and retrograde (bottom) segmental velocities and 
bioRxiv preprint doi: https://doi.org/10.1101/2020.09.27.315846; this version posted September 28, 2020. The copyright holder for this preprint (which was not certified by peer review) is the author/funder. All rights reserved. No reuse allowed without permission.

segmental run length of PrPWT- and PrP PG14-EGFP vesicles. ${ }^{* *} p<0.01,{ }^{* * *} p<0.001$, Kolmogorov-Smirnov test. 


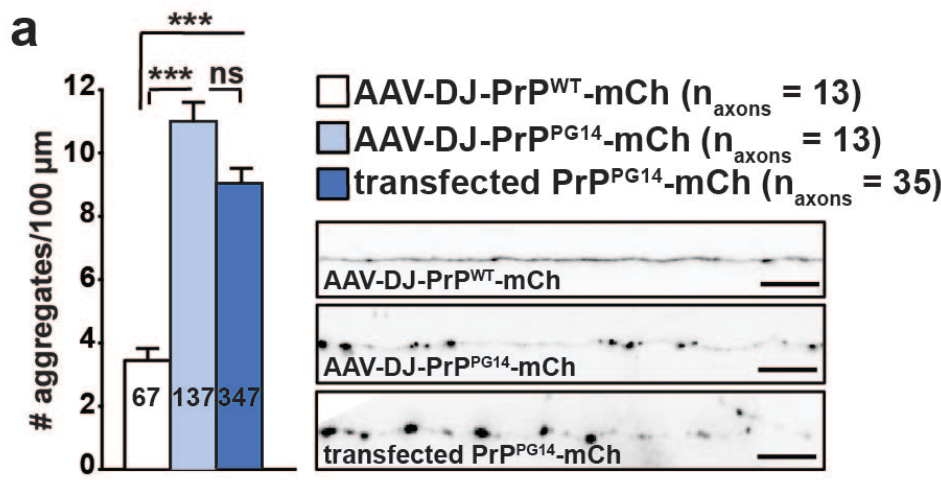

b
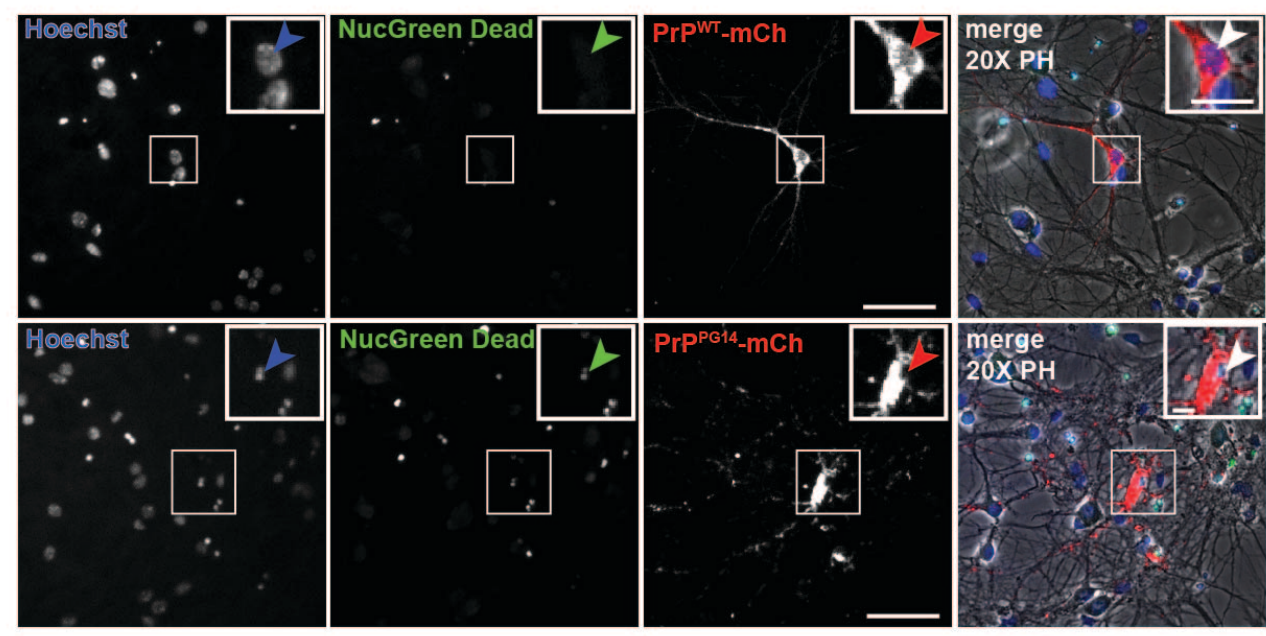

C

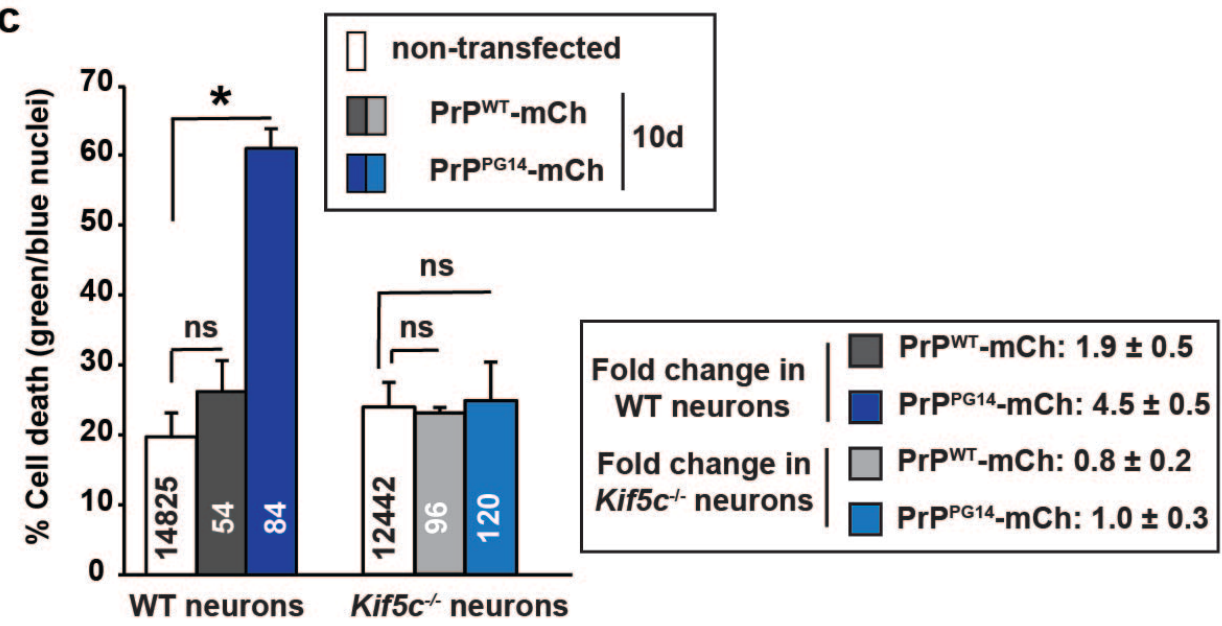

\section{Supplementary Figure 10. Kinesin-1-dependent endoggresomes impair calcium} influx and accelerate cell death of $\mathrm{PrP}^{\mathrm{PG} 14}$ neurons. Related to Figure 8. a,

Quantitation of aggregate densities (left) and representative images (right) of axons from neurons transduced with AAV-DJ-PrP ${ }^{\mathrm{PG} 14}$-mCh. $\mathrm{N}_{\text {aggregates }}$ are shown inside bars. Scale 
bar $=10 \mu \mathrm{m} . \mathbf{b}$, Representative images of hippocampal neurons expressing PrP ${ }^{W T}$ - or PrP ${ }^{\mathrm{PG} 14}$-mCh and stained with Hoechst and NucGreeen Dead. Arrowheads point to alive (top panels) and dead (bottom panels) neurons. Insets are enlarged in the respective top right corners of each panel. Scale bars on main figures $=100 \mu \mathrm{m}$. Scale bars on insets $=$ $20 \mu \mathrm{m} . \mathbf{c}$, Quantitation of percentage cell death from (b). $\mathrm{N}_{\text {cells }}$ are shown inside bars. Fold changes between indicated conditions are indicated on boxed panel (right). All values are shown as mean \pm SEM. ${ }^{*} p<0.05,{ }^{* * *} p<0.001$, ns $=$ non-significant, KruskalWallis test. 


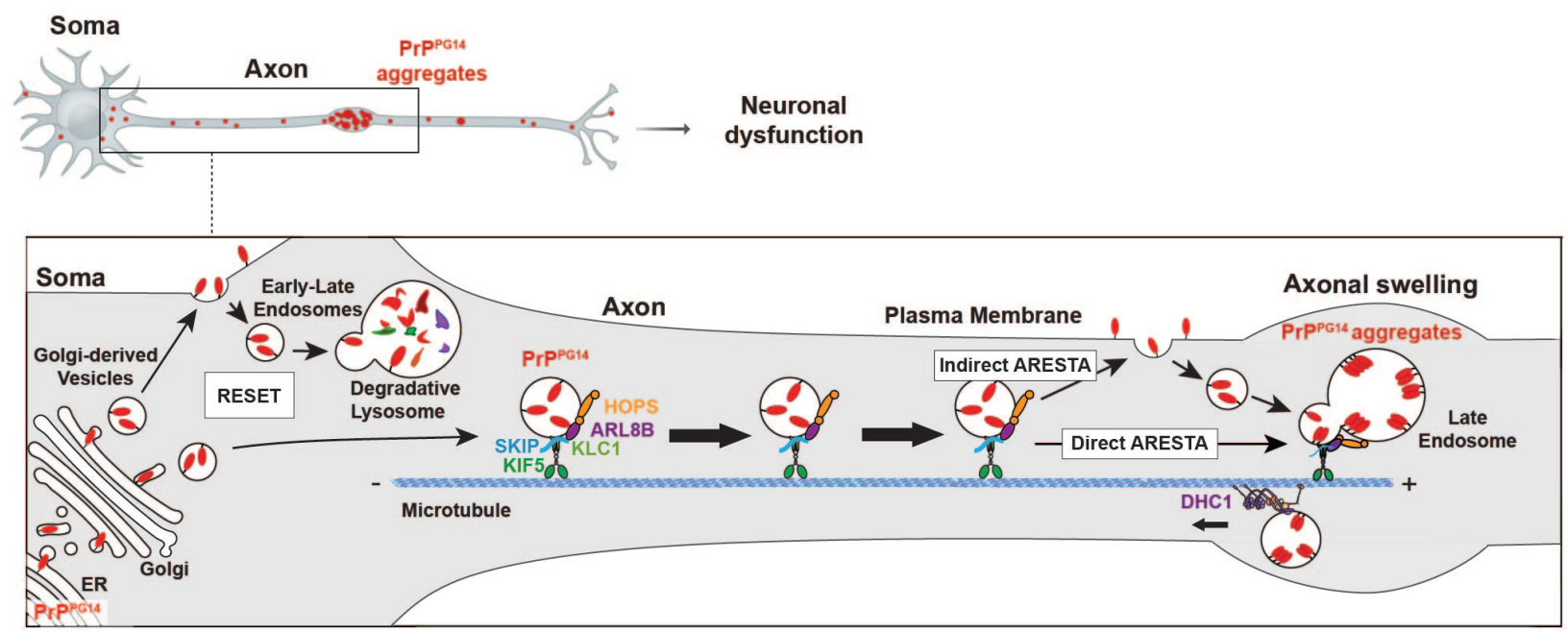

\section{Supplementary Figure 11. Neuronal endo-lysosomal trafficking RESET and}

\section{ARESTA pathways that clear misfolded PrP or form endoggresomes}

Model showing endosomal pathways governing mutant PrP degradation versus

aggregation in neurons. RESET acts in the soma to degrade $\mathrm{PrP}^{\mathrm{PG} 14}$ via the cell surface.

Arl8b/kinesin-1/SKIP/HOPS earmarks $\mathrm{PrP}^{\mathrm{PG} 14}$ vesicles as endosomes and drives their axonal entry and toward ARESTA-dependent aggregation. Aggregation occurs following indirect targeting of $\mathrm{PrP}^{\mathrm{PG} 14}$ to late endosomes via the axonal cell surface, via its direct homotypic fusion, or both. Dynein-mediated retrograde transport and axonal degradative capacity are impaired in axons thus promoting the maintenance of aggregates in axons. 
bioRxiv preprint doi: https://doi.org/10.1101/2020.09.27.315846; this version posted September 28, 2020. The copyright holder for this preprint (which was not certified by peer review) is the author/funder. All rights reserved. No reuse allowed without permission.

\section{DATA AVAILABILITY}

The datasets generated during and/or analyzed during the current study are available from the corresponding author on reasonable request. 